\title{
A CRITICAL REVIEW OF HEALTH IMPACT ASSESSMENTS IN ONTARIO'S NUCLEAR INDUSTRY
}

by

\author{
William Mueller
}

Bachelor of Science (Honours), McMaster University, 2007

\author{
A thesis \\ presented to Ryerson University \\ in partial fulfillment of the \\ requirements for the degree of \\ Master of Applied Science \\ in the program of \\ Environmental Applied Science and Management
}

Toronto, Ontario, Canada, 2010

(c) William Mueller, 2010 


\section{AUTHOR'S DECLARATION}

I hereby declare that I am the sole author of this thesis.

I authorize Ryerson University to lend this thesis to other institutions or individuals for the purpose of scholarly research.

William Mueller

I further authorize Ryerson University to reproduce this thesis by photocopying or by other means, in total or in part, at the request of other institutions or individuals for the purpose of scholarly research.

William Mueller 
"Numbers are seductive: they imply a degree of accuracy and certainty that is often unjustified."

(Applegate, 1995:1671) 


\section{ABSTRACT}

A Critical Review of Health Impact Assessments in Ontario's Nuclear Industry

William Mueller

Master of Applied Science

Environmental Applied Science and Management

September 2010

Ryerson University

Risk is central to the health effects of nuclear power plants. The regulator in Canada, the Canadian Nuclear Safety Commission (CNSC), claims to employ international best practices and risk-informed decision-making to ensure Canadian plants are among the safest in the world. Environmental Assessment (EA), required for operating license approval, is used to determine whether risks to pubic health, both chronic and catastrophic, are within acceptable limits. The main objective of this thesis is to establish Health Risk Assessment (HRA) best practices, approximated by the degree of concordance among HRA authorities, and use these concepts to evaluate EAs of recent nuclear power projects. The extent of compliance would ultimately reveal the CNSC's commitment to protecting public health and safety. It is concluded from the review of six such EAs that the CNSC is falling short of best practices, ultimately approving projects without an accurate estimation of risk. 


\section{ACKNOWLEDGMENTS}

There were a number of individuals who assisted me in this research:

Dr. Ronald Pushchak, for providing endless guidance and support. This research benefitted immensely from the innumerable ideas generated throughout the many lengthy discussions.

Sarah Watt, CNSC, and Jennifer Kirkaldy, SENES Consultants Ltd., for supplying several EA documents.

All of the research participants, whose input proved invaluable to this research.

David Forgione, for his unparalleled diagrammatic expertise.

Ryerson University, for providing funding via the Ryerson Graduate Award.

Victoria McCulloch, for maintaining my sanity and well-being throughout the entire research process.

And, of course, my graduating cohort at Ryerson University, with whom I have shared many times of enlightenment and entertainment. 


\section{TABLE OF CONTENTS}

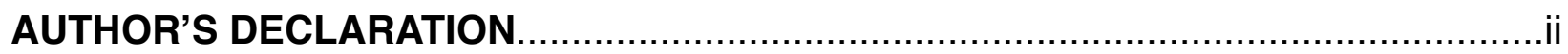

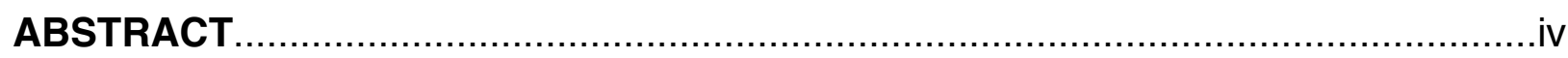

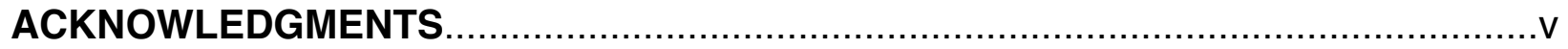

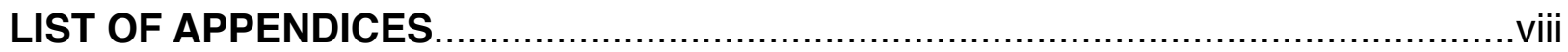

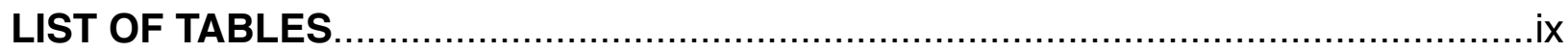

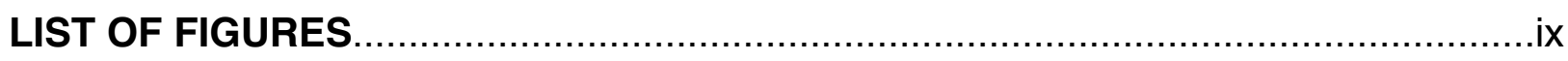

LIST OF ABBREVIATIONS

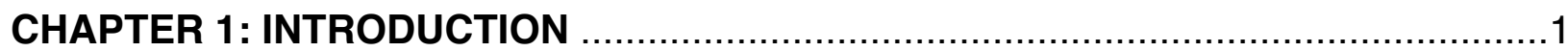

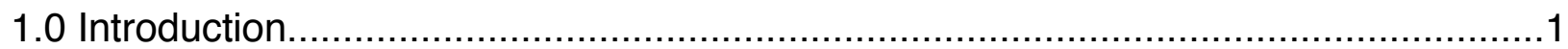

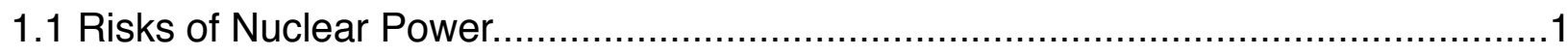

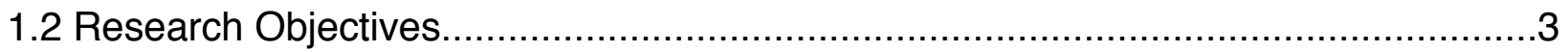

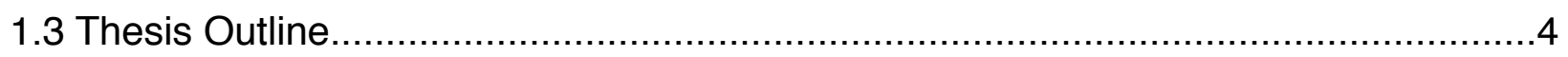

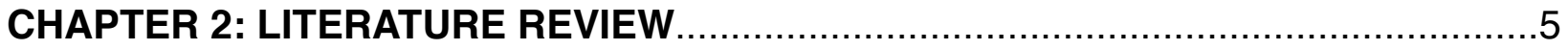

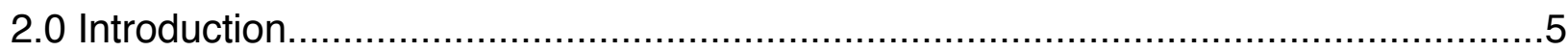

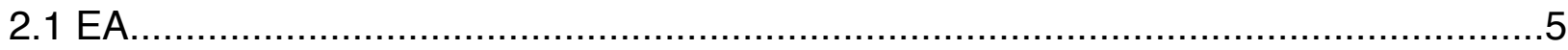

2.1.1 Early EA

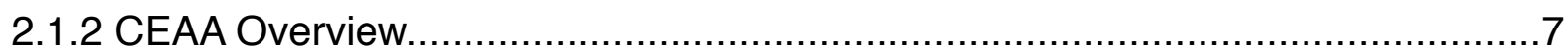

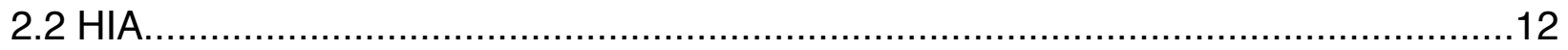

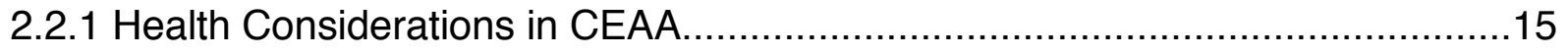

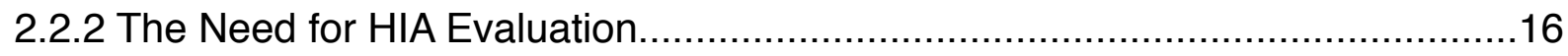

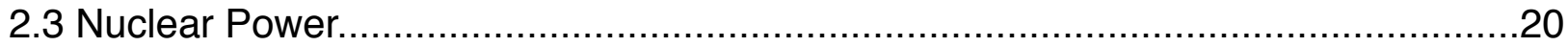

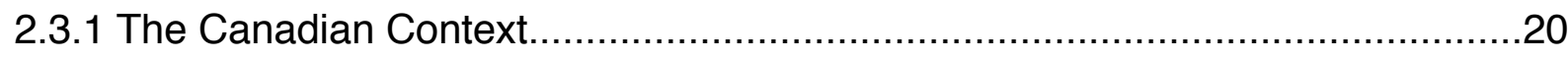

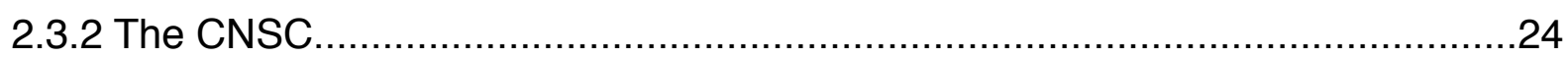

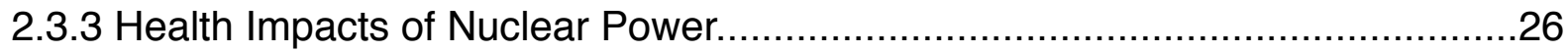

2.3.3.1 Chronic Health Impacts..................................................................2 


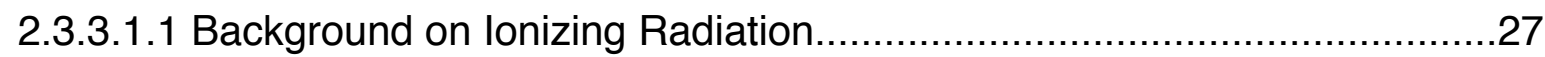

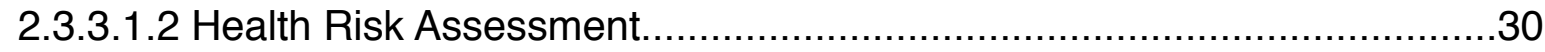

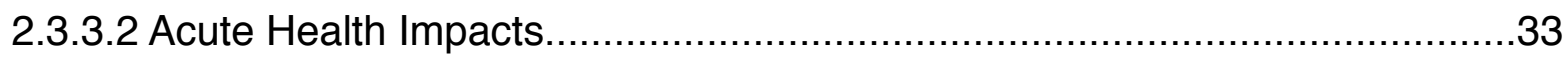

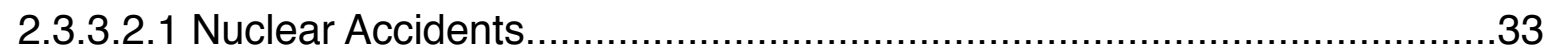

2.3.3.2.2 Probabilistic Risk Assessment..........................................................

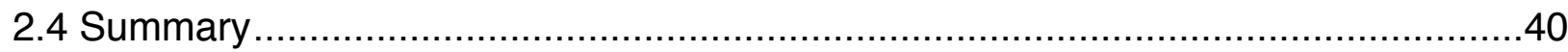

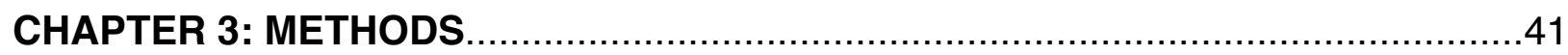

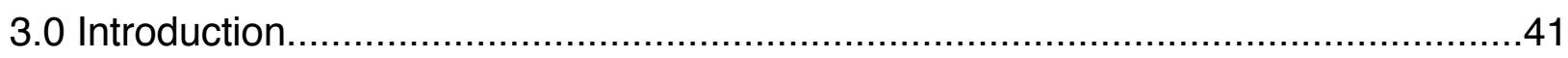

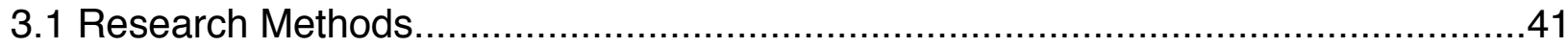

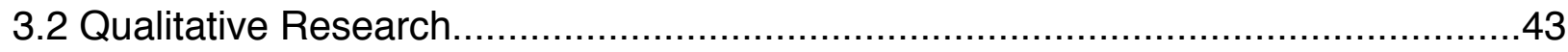

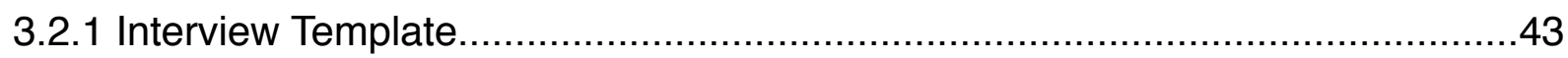

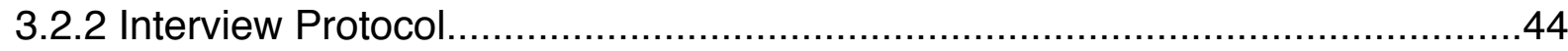

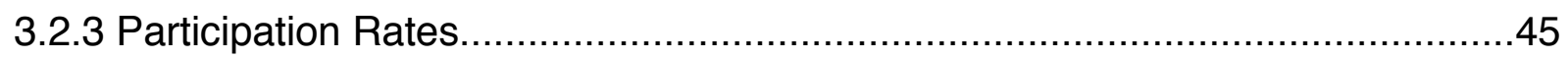

3.3 Framework Design: A ‘Risk Assessment' Approach..........................................48

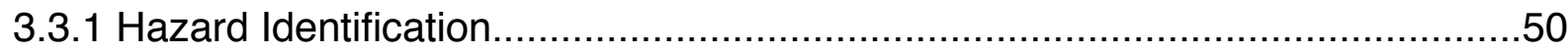

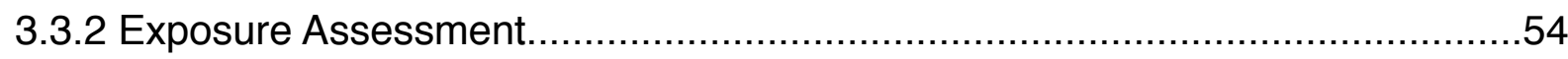

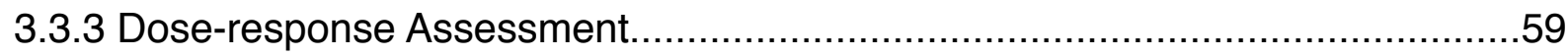

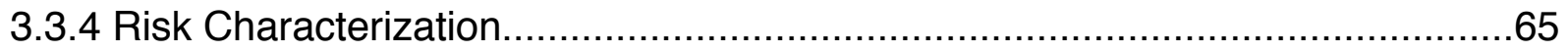

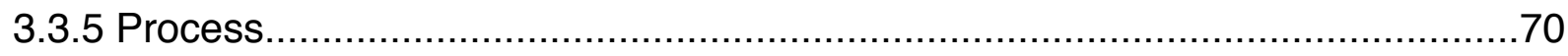

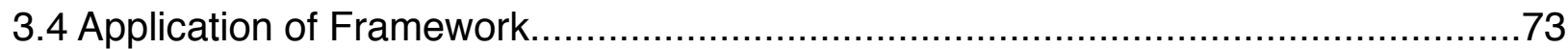

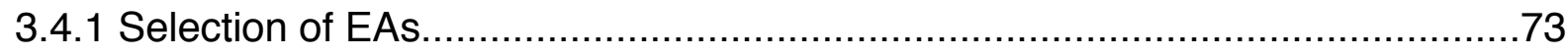

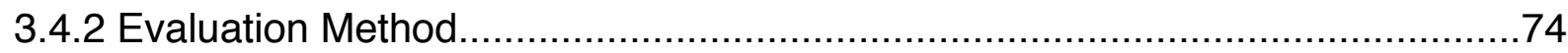

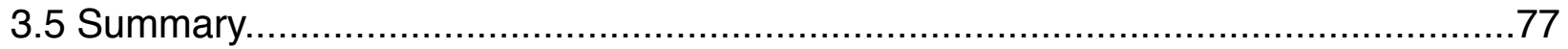

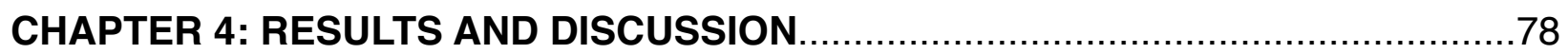

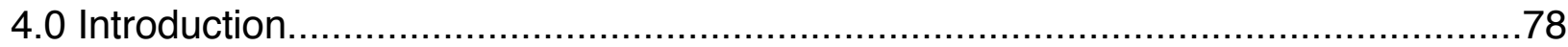

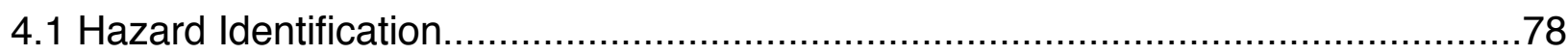

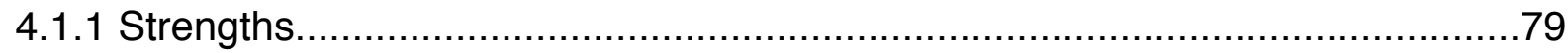

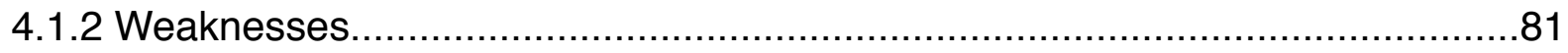

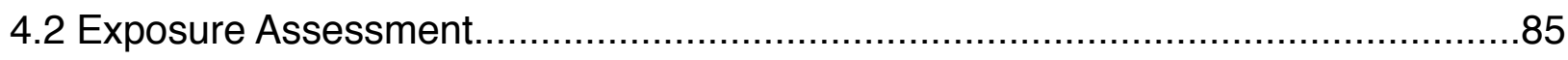




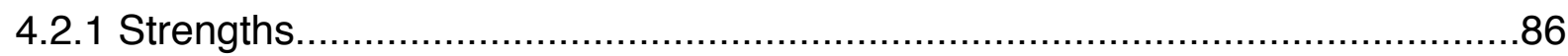

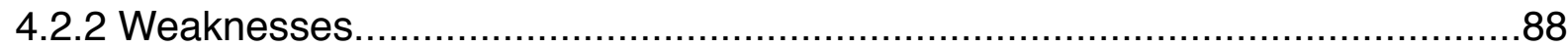

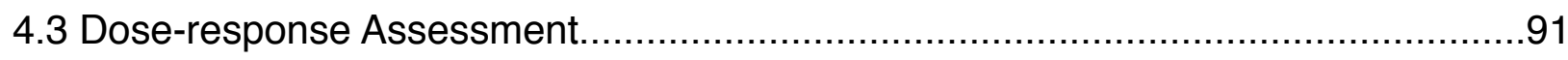

4.3.1 Strengths

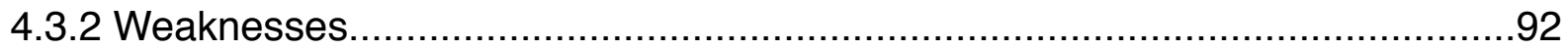

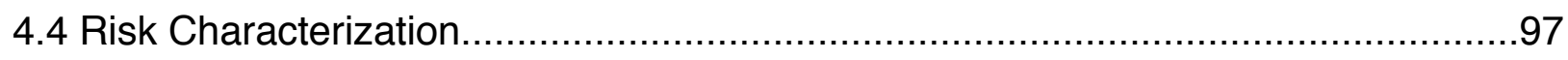

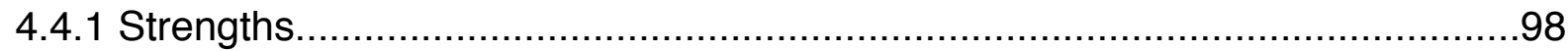

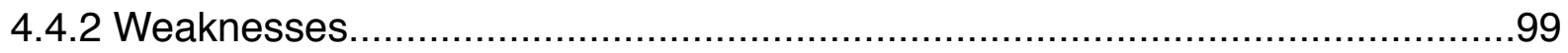

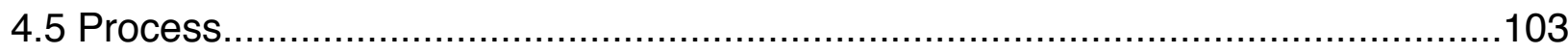

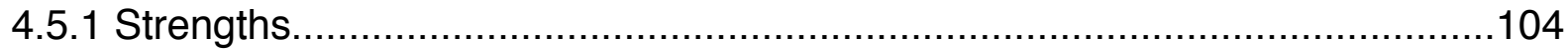

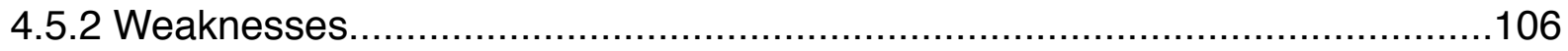

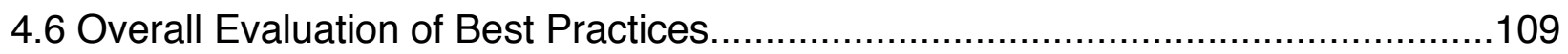

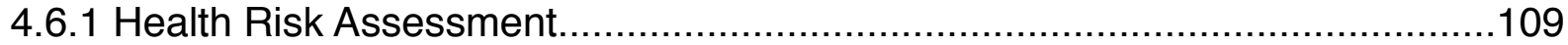

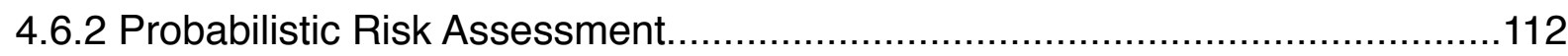

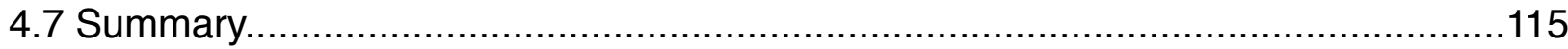

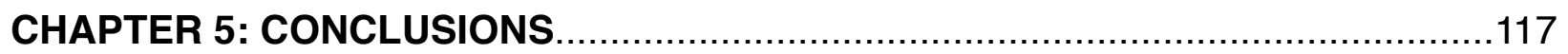

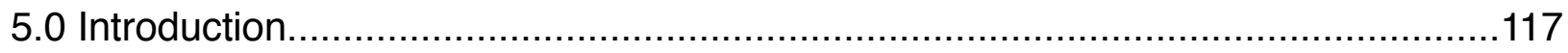

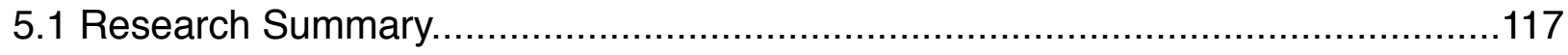

5.1.1 Compliance with Best Practices...........................................................119

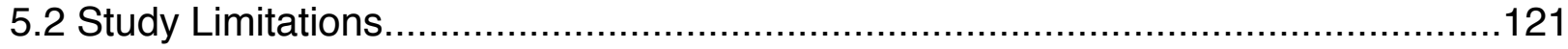

5.3 Research Contribution and Future Application..............................................122

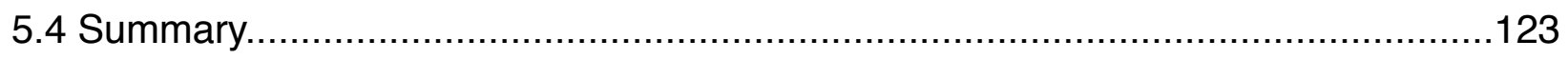

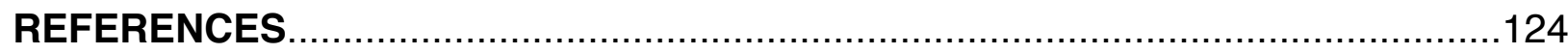

\section{LIST OF APPENDICES}

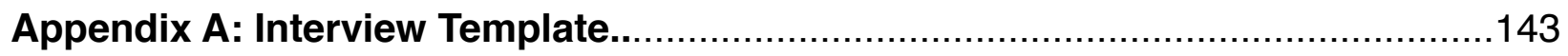

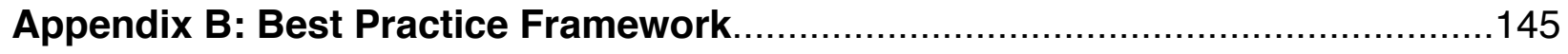




\section{LIST OF TABLES}

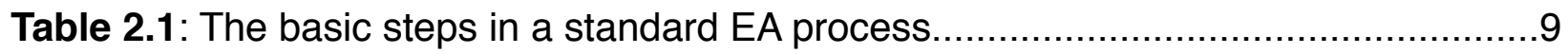

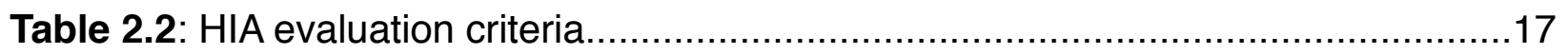

Table 2.3: Criteria to evaluate HRA integration in EA.............................................18

Table 2.4: The fundamental steps of an HRA........................................................

Table 3.1: A summary of the best practice criteria derived for the Hazard Identification section, as outlined in the preceding discussion........................................54

Table 3.2: A summary of the best practice criteria derived for the Exposure Assessment section, as outlined in the preceding discussion........................................59

Table 3.3: A summary of the best practice criteria derived for the Dose-response Assessment section, as outlined in the preceding discussion......................65

Table 3.4: A summary of the best practice criteria derived for the Risk Characterization section, as outlined in the preceding discussion........................................69

Table 3.5: A summary of the best practice criteria derived for the Process section, as outlined in the preceding discussion.....................................................

Table 4.1: The results of the Hazard Identification section.........................................79

Table 4.2: The results of the Exposure Assessment section......................................86

Table 4.3: The results of the Dose-response Assessment section...............................91

Table 4.4: The results of the Risk Characterization section..........................................98

Table 4.5: The results of the Process section.......................................................104

Table 4.6: The results of the five sections of the best practice framework..................110

Table 4.7: The PRA-specific evaluation criteria..................................................113

Table 4.8: The results from the PRA-specific evaluation...........................................113

\section{LIST OF FIGURES}

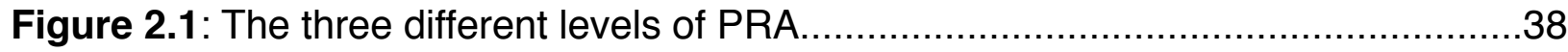

Figure 3.1: The methods used to achieve the research objectives.............................42

Figure 3.2: The number of potential participants from different areas of expertise........47

Figure 3.3: The number of interviewees from different areas of expertise....................47 


\section{LIST OF ABBREVIATIONS}

AECB: Atomic Energy Control Board

AECL: Atomic Energy of Canada Limited

ALARA: As Low As Reasonable Achievable

BAPRA: Bruce A Probabilistic Risk Assessment

BBRA: Bruce B Risk Assessment

BP: Bruce Power

Bq: Becquerel

CANDU: Canada Deuterium Uranium

CEAA: Canadian Environmental Assessment Act

CERRIE: Committee Examining Radiation Risks from Internal Emitters

CNSC: Canadian Nuclear Safety Commission

DCF: Dose Conversion Factor

EA: Environmental Assessment

EARP: Environmental Assessment and Review Process

EIA: Environmental Impact Assessment

EIS: Environmental Impact Statement

EPRC: Ex-Plant Release Category

FA: Federal Authority

HIA: Health Impact Assessment

HPA: Health Protection Agency

HRA: Health Risk Assessment

IAEA: International Atomic Energy Agency

ICRP: International Committee on Radiological Protection

IIPA: Independent Integrated Performance Assessment

IP: Indian Point

IPSP: Integrated Power System Plan

LNT: Linear No Threshold

LOCA: Loss of Coolant Accident

MEI: Maximally Exposed Individual

NAS: National Academy of Sciences 
NEA: Nuclear Energy Agency

NEPA: National Environment Policy Act

NRU: National Research Universal

NSCA: Nuclear Safety and Control Act

OH: Ontario Hydro

OPA: Ontario Power Authority

OPG: Ontario Power Generation

PARA: Pickering A Risk Assessment

PBRA: Pickering B Risk Assessment

PRA: Probabilistic Risk Assessment

PSA: Probabilistic Safety Assessment

RA: Responsible Authority

RSS: Reactor Safety Study

SIA: Social Impact Assessment

Sv: Sievert

TMI: Three Mile Island

TSD: Technical Support Document

UNSCEAR: United Nations Scientific Committee on the Effects of Atomic Radiation

USEPA: United States Environmental Protection Agency

USNRC: United States Nuclear Regulatory Commission

WHO: World Health Organization 


\section{CHAPTER 1: INTRODUCTION}

\subsection{Introduction}

For the first time since the 1970s, the construction of new nuclear installations is being considered in Ontario. The approval process is governed by Environmental Assessment ${ }^{1}(E A)$, which has traditionally not been geared toward human health. Recent efforts in Canadian EA have been to embed a parallel Health Impact Assessment (HIA) process into EA, but uncertainties remain over methods and impact prediction. Risk, both chronic and catastrophic, is central to the health effects of nuclear power plants. The research in this thesis aims to critically examine the assessment of health risks through the HIA process of both completed and current nuclear projects in Ontario, with the overarching goal of determining whether public health is being sufficiently protected.

\subsection{Risks of Nuclear Power}

Nuclear power involves both chronic and catastrophic risks to public health. Releasing small amounts of radiation into the environment is part of the normal operation of nuclear power plants (Galeriu et al., 2003), and has been suspected by many researchers to be the cause of excess cancer incidences (Mangano, 2006; Baker and Hoel, 2007; Kaatsch et al., 2008). The risk of catastrophic accidents has already been demonstrated by the Chernobyl disaster in April 1986, which led to a host of documented health detriments (Fairlie and Sumner, 2006). Another aspect of risk has been added in the wake of 9/11: threats of terrorism. Indeed, nuclear plants, which

\footnotetext{
1 EA is also known as Environmental Impact Assessment (EIA), but the former is more common in the Canadian context and will thus be used in this thesis.
} 
concentrate more lethal material than anything else in our society (Perrow, 2007), are considered a particularly susceptible target:

Throughout the world I think AI Qaeda is looking for those vulnerabilities in facilities and stockpiles in different countries that would allow them to obtain the by-products of nuclear reactors and materials that they can use (Brennan, 2010).

Additionally, a number of experts have expressed concern over the possibility of a malevolent act causing a large-scale radioactive release, specifically from the spent fuel storage pools (Alvarez, 2002; Thompson, 2003; Steinhäusler, 2009).

The nuclear regulator in Canada is the Canadian Nuclear Safety Commission (CNSC), which purports to function at arm's length from the federal government ${ }^{2}$ (CNSC, 2008b). Following the lead of the United Stated Nuclear Regulatory Commission (USNRC), the CNSC has adopted a "risk-informed approach to regulatory strategies, regulations and licensing requirements" (CNSC, 2005a:17). Catastrophic and chronic risks in the nuclear industry are managed by two regulatory tools to ensure public health risks are within acceptable levels: Probabilistic Risk Assessment (PRA) and Health Risk Assessment (HRA), respectively. A review in the US concluded that licensing decisions were being based on inherently flawed risk assessments (Lochbaum, 2004). No parallel study has yet been conducted in Canada. Despite the CNSC's commitment to international best practices (CNSC, 2008b), Perrow (1999) "argued that because of the complexity of these [nuclear] plants and their tight coupling, serious accidents are inevitable even with the best management practices and attention to safety" (Perrow, 2007:172) [italics in original]. Furthermore, the refurbishment and

${ }^{2}$ Recent events have brought into question the independence of the CNSC, see subsection 2.3.2. 
continued operation of aging plants present even greater risks from age-related detriment (Lochbaum, 2004). A critical examination of the nuclear power plant EA approval process can reveal whether the CNSC is indeed employing best practices; such compliance would be necessary to ensure the public is being protected from undue health and safety risks.

\subsection{Research Objectives}

Several HIA researchers have proposed recommendations to improve the consideration of health impacts in EA. Cole and Fielding (2007) prescribed the use of standardized checklists of health determinants, in addition to consulting experts and stakeholders, to establish the most important potential impacts of a project. The BMA (1999:103) advocated a similar viewpoint: "a systematic review of the health hazards associated with developments in each sector should be carried out in order to produce a comprehensive checklist which could be used to guide developers and planning authorities". The goal of this research is to devise such a nuclear power-specific framework and subsequently use it to evaluate nuclear related EAs in Ontario. In essence, this thesis strives to answer the question:

Do recent HIAs for nuclear power projects conform to International Best Practices ${ }^{3}$, as indicated in the CNSC mandate?

\footnotetext{
3 The concept of "best practices" has gained recent popularity in a variety of settings, yet there remains little consensus on a precise meaning (Zaring, 2006). Here, the term is meant to represent ideal HIA methods for nuclear power projects: those principles which are dominant in the literature and substantiated by experts.
} 
In order to answer this question, the following research objectives are accomplished:

1. Establish HIA best practices for nuclear power projects from authoritative sources on HIA, HRA and PRA;

2. Create an evaluation framework based on consistently identified best practices;

3. Apply the framework to released Environmental Impact Statements (EISs) ${ }^{4}$ of nuclear power projects in Ontario;

4. Determine the extent to which best practices have been achieved and recognize any significant deficiencies.

\subsection{Thesis Outline}

This first introductory chapter has presented background information to establish a research focus. Chapter 2 provides a more in depth discussion of the EA and HIA process, as well as a review of the human health issues pertinent to nuclear power. Chapter 3 explains the qualitative research methods, ultimately leading to the derivation of the best practice evaluation framework. With the framework developed, the EIS selection process and review protocol are described. Chapter 4 examines the strengths and weaknesses identified in each section and provides an overall comparison of the treatment of chronic and catastrophic risks. Chapter 5 then summarizes significant conclusions from the EIS reviews. Lastly, limitations of the study are recognized, as well as future implications of the research.

\footnotetext{
${ }^{4} \mathrm{An}$ EIS is the written document that provides a detailed description of the studies and results of the EA.
} 


\section{CHAPTER 2: LITERATURE REVIEW}

\subsection{Introduction}

The aim of this research is to devise an HIA best practice framework for the purposes of evaluating EISs of nuclear power projects. Some further background information on several issues is needed before this objective may be realized. This chapter first provides a brief summary of the EA and HIA process, before examining the treatment of "health" in the Canadian Environmental Assessment Act (CEAA). This description then leads into a review of HIA evaluation efforts. With the background on HIA established, the focus turns to the Canadian nuclear power industry. Finally, chronic and acute health impacts of nuclear power are outlined, as well as the corresponding regulatory tools (HRA and PRA, respectively) employed to quantify such risks.

\subsection{EA}

\subsubsection{Early EA}

The United States was the first nation to create EA legislation with the enactment of the National Environment Policy Act (NEPA) in 1969. The primary objective of EA is to evaluate projects prior to construction and attempt to increase positive impacts, while mitigating any negative impacts on the biophysical and socio-economic environment. EA requires proponents to demonstrate that projects are not likely to cause significant harm to the environment prior to development (Noble, 2006). However, it would be naïve to believe this legislation was for the sole purpose of safeguarding nature. 
Instead, EA would protect the well-being of the public through maintaining a safe and healthy environment. A leading author of NEPA expanded on this idea:

When we speak of the environment, basically, we are talking about the relationship between man and these physical and biological and social forces that impact upon him. A public policy for the environment basically is not a public policy for those things out there. It is a policy for people (Senator Henry Jackson (1969) in Bhatia and Wernham, 2008).

Other countries soon followed the US's lead and formed their own EA policies; the Canadian federal EA process took the form of the Environmental Assessment and Review Process (EARP) in 1973 (Noble, 2006). This approach was based upon the notion of proponent self-assessment, ultimately resulting in project proponents applying the requirements of $E A R P$ at their own discretion. In 1984, EARP was formally registered as a "guidelines order" in an attempt to ensure improved EA compliance among federal authorities, yet large projects often continued to circumvent EA scrutiny (Gibson and Hanna, 2005). It was not until two precedent-setting court cases that the Guidelines Order was determined to be legally binding. In 1989 a federal court judge quashed a license for the Rafferty-Alameda Dam in Saskatchewan, as no federal EA was conducted. Likewise, three years later the Supreme Court of Canada ruled that an EA be conducted for the proposed Oldman River Dam in Alberta (Hazell, 1999). These two court cases were central to the formation of $C E A A$, finally being promulgated in 1995.

CEAA now provides a legal mandate for EA when a federal authority (FA) is the proponent of a given project or alternatively, takes any the following courses of action: making or authorizing a payment for the project, transferring federal lands for the 
purposes of carrying out the project or issuing a license or permit to enable the project (CEAA, s. 5(1)). If an EA is indeed warranted, the respective FA assumes the role of responsible authority (RA) to "ensure that the environmental assessment is conducted as early as is practicable in the planning stages of the project and before irrevocable decisions are made" (CEAA, s.11(1)).

\subsubsection{CEAA Overview}

A defining feature of $C E A A$ is that it distinguishes between varying degrees of assessment, as determined by the potential impacts of a project type. The most basic level is a screening, followed by a comprehensive study for larger projects. If a more detailed assessment is needed than that of a comprehensive study, the Minister of the Environment may upgrade the EA to a review panel. Alternatively, involved parties may wish to opt for a type of EA known as a mediation, whereby the Minister selects a mediator to resolve any outstanding issues (Herring, 2005). In practice, however, mediation is quite rare (Yap, 2003).

The important characteristics of screenings, which constitute $99 \%$ of all EAs, include mandatory 15 day public comment periods for screening reports (Herring, 2005), RA discretion as to whether to provide the public with information on the scope of the project (CEAA, s.18 (3a)), and possible upgrading to a comprehensive study if the project is either likely to cause significant adverse effects or is the subject of considerable public concern (CEAA, s.20 (1c(i))). Comprehensive studies, in addition to 
mandating public consultation on the scope of the project ${ }^{5}$ and ensuring a 30 day comment period on the comprehensive study report ${ }^{6}(C E A A$, s.21(1)), must also address both the purpose and alternatives of the project (CEAA, s.16 (2a-c)). Another key difference is that after the initial public consultation, the RA must report to the Minister on the scope, public concerns and potential for adverse environmental effects of the project. If warranted, the Minister may refer the EA to a review panel (CEAA, s.21 (2a)); otherwise, the comprehensive study will proceed. If a review panel is necessary, the Minister will select a panel of experts who "must be unbiased and free from conflicts of interest" (Herring, 2005:238), to conduct an independent assessment and hold separate public hearings. Upon completion, the panel issues a report on the various findings of the EA, which is then used by the RA to decide final project approval (Herring, 2005).

Regardless of the EA type prescribed by CEAA, the procedure always follows a similar sequence (outlined in Table $2.1^{7}$ ) and results in the formation of an EIS. The differences of each type illustrate how the process evolves from being relatively constrictive in screening level assessments to being more extensive and open in panel reviews.

\footnotetext{
${ }^{5}$ Note that even though the RA may provide scoping information to the public during a screening, this is not the same as consulting the public, which takes place in a comprehensive study; the latter entails a chance for public feedback.

${ }^{6}$ Although the names of the documents prepared during a screening and comprehensive study may vary, they are both EISs and will be referred to as such.

${ }^{7}$ Notice the double usage of the term "screening" here: (1) the most basic level of EA and (2) the process by which it is determined if a project requires an EA.
} 


\begin{tabular}{|l|l|}
\hline EA Step & Description \\
\hline Project Description & $\begin{array}{l}\text { Description of the proposed action, including its alternatives, } \\
\text { and details sufficient for an assessment. }\end{array}$ \\
\hline Screening & $\begin{array}{l}\text { Determination of whether the action is subject to an EIA under } \\
\text { the regulations or guidelines present, and if so what type or } \\
\text { level of assessment is required. }\end{array}$ \\
\hline Scoping & $\begin{array}{l}\text { Delineation of the key issues and the boundaries to be } \\
\text { considered in the assessment, including the baseline } \\
\text { conditions and scoping of alternatives. }\end{array}$ \\
\hline $\begin{array}{l}\text { Impact prediction } \\
\text { and evaluation }\end{array}$ & $\begin{array}{l}\text { Prediction of environmental impacts and determination of } \\
\text { impact significance. }\end{array}$ \\
\hline $\begin{array}{l}\text { Impact } \\
\text { management }\end{array}$ & $\begin{array}{l}\text { Identification of impact management and mitigation strategies, } \\
\text { and development of environmental management of protection } \\
\text { plans. }\end{array}$ \\
\hline $\begin{array}{l}\text { Review and } \\
\text { decision }\end{array}$ & $\begin{array}{l}\text { Technical and public review of the Environmental Impact } \\
\text { Statement (EIS) and related documents, and subsequent } \\
\text { recommendation as to whether the proposed action should } \\
\text { proceed and under what conditions. }\end{array}$ \\
\hline $\begin{array}{l}\text { Implementation and } \\
\text { follow-up }\end{array}$ & $\begin{array}{l}\text { Implementation of project and associated management } \\
\text { measures }\end{array}$ \\
\hline
\end{tabular}

Table 2.1. The basic steps in a standard EA process (Adapted from Noble, 2006:13).

Although Table 2.1 provides a good outline of the EA process, some key issues of EA (and CEAA) should be emphasized before delving into a discussion of HIA. The first step in the EA process is the preparation of a project description by the proponent, usually detailing the proposed actions of a project and possible alternatives. This information is used during the screening stage to determine whether an EA is required. Generally, projects that include physical work are subject to EA and are listed in the CEAA Inclusion List. Conversely, projects that do not entail significant adverse environmental effects are exempted from $\mathrm{EA}$, according to the CEAA Exclusion List. The Comprehensive Study List dictates which projects must be subjected to a 
comprehensive study EA and includes projects pertaining to national parks, oil and gas, and nuclear power, among others (CEAA, 1994). Additionally, each province has its own EA legislation; both federal and provincial EAs are sometimes required, depending on whether the nature of the project falls under federal or provincial jurisdiction. Although some projects, for instance nuclear power plants, automatically fall under federal legislation.

A detailed scoping stage is critical for ensuring a sound EA process. It is through scoping that the various issues in need of assessment are identified, thus producing the terms of reference for the EA studies (Hanna, 2005). Mulvihill (2003) listed the features of an ideal scoping process: early application, public input, identification of alternatives, and a focus on key issues. Instead of proponents and consultants carrying out scoping in private, Mulvihill recommended seeking input from a range of stakeholders to improve the EA process.

Making decisions on which developments are environmentally benign and publicly supported is one of the main tenets of EA. To make such an informed decision, a comprehensive analysis of alternatives must be completed. However, Steinemann (2001) posited that the EA process is typically initiated after a proponent has already settled on a specific project, and that environmentally superior alternatives have been disregarded before serious consideration can be given via EA. With regard to CEAA, recall that alternatives are not required in screenings, which represent the vast majority of EAs in Canada (CEAA, s.16 (2b); Herring, 2005).

The heart and soul of EA is the prediction of environmental impacts and consequent determination and communication of impact significance. Yet, the concept 
of "significance" remains contentious; often, it may be dependent upon the values of a particular society at a specific time (Wood, 2008). In other jurisdictions, for example, the US, significance is evaluated by court interpretation under NEPA (Harvey, 1990). The identification of environmental impacts must correspond with mitigation and management strategies. All of this information is then compiled into an EIS and presented to the public and decision makers. The consultation with the public in this phase, and in fact, throughout the EA process, is a fundamental component of EA. Unfortunately, the public participation process in Canada has been heavily criticized, even after 30 years of experience (Doelle and Sinclair, 2006). Several barriers hindering effective public participation have been identified, including a lack of public resources, few opportunities to participate, and perhaps most importantly, the public's overwhelming sense that participation efforts are no more than futile attempts to alter the course of an undertaking (Diduck and Sinclair, 2002).

Taking into consideration the significance of predicted adverse environmental effects, the proposed mitigation strategies, and follow-up activities, the RA makes a decision whether to approve the EA, approve it conditionally, or reject it outright ${ }^{8}$. Finally, CEAA prescribes the establishment of follow-up activities, the objectives of which are two-fold: to confirm the predictions made during the EA and to monitor the effectiveness of mitigation procedures (Noble, 2006).

\footnotetext{
8 In the case of review panels, the RA has to additionally consider the recommendations of the panel and the comments from the Minister.
} 


\subsection{HIA}

Fundamentally, HIA investigates and predicts possible health impacts of a policy, programme or project in order to devise recommendations for a final decision, much like an EA does for environmental impacts (Quigley and Taylor, 2004). Similarly, an HIA may be instrumental in encouraging any positive effects of an undertaking, while minimizing negative impacts. This is often the case, as development has been regarded as a double-edged sword (Davies and Sadler, 1997). For example, a project may provide more employment for a population and create a better standard of living; however, the project may also entail increased traffic, which could further degrade air quality and cause more accidents. Additionally, an HIA can identify and address specific populations who may bear a disproportionate number of negative project impacts (Harris et al., 2009). If an HIA succeeds in protecting these vulnerable populations, the assumption is that the greater public would also be safeguarded (BMA, 1999).

The World Health Organization (WHO) definition of health is very inclusive: "a state of complete physical, mental and social well-being and not merely the absence of disease or infirmity" (Health Canada, 2004a:4). As such, a broad range of health determinants have been identified in a number of categories: biological factors, personal and family environments, social and physical environments, as well as public services and policy (Birley, 2003). The interaction between one's social status and physical health is complex, but HIA practitioners now recognize the fact that there must be an analysis of the social determinants ${ }^{9}$ of health, such as income and social status, social support networks, employment and working conditions (Kwiatkowski and Ooi, 2003).

\footnotetext{
9 Traditionally, social impacts have been addressed in a Social Impact Assessment (SIA), but recent work suggests combining SIA and HIA (Rattle and Kwiatkowski, 2003).
} 
Yap (2003) combined social and health factors to create an inclusive impact assessment framework, for the purpose of improving sustainable development in Canadian EA. Ensuring a broad list of human health determinants is necessary if an EA is to honour the WHO definition of health.

A considerable number of efforts have been dedicated to establishing an accepted methodology for HIA. Cole and Fielding (2007) discussed three main categories of HIA: quantitative/analytic, participatory, and procedural. The first approach combines possible health effects from a literature review with information on a target population to estimate potential impacts; this technique is strongly related to HRA $^{10}$. Conversely, the participatory technique focuses on community-based health promotion and is mostly appropriate for broader policies. The third method is quite similar to the quantitative/analytic approach, but is often associated with EA and makes use of standardized procedures. Aside from these broad categories, reviews of completed HIAs have not yet yielded a model methodology. Mindell et al. (2008) investigated $27 \mathrm{HIAs}$, but, although identifying various methodological strengths and weaknesses, did not select a preferred approach. They did note, however, that higher quality HIAs tend to be associated with the adoption of a holistic definition of health, sufficient funding, and project-level assessments (when compared to those of policies $\left.{ }^{11}\right)$. Cole et al. (2005:383) concurred with these findings and offered a similar perspective:

\footnotetext{
10 HRA is discussed in detail in subsection 2.3.3.1.2.

11 This discouraging trend has been observed for EAs of important policies in Ontario. Most notably, the Integrated Power System Plan (IPSP), which is estimated to require $\$ 60$ billion in capital investments, was exempted from Ontario's provincial EA requirements in June 2006. Instead of using EA to investigate the broad environmental and social implications of Ontario's future electricity plan, the IPSP is being evaluated by the Ontario Energy Board in terms of rates, costs, and fairness (Miller, 2008).
} 
Although different kinds of criteria need to be employed to assess different kinds of situations, making it impractical to use a single set of criteria, such as those used for evaluating evidence in evidence-based research reviews, it is essential that the process for gathering and evaluating evidence is explicit, transparent, and balanced.

The importance of effective public participation, similar to EA, is an underlying theme in HIA (BMA, 1999). True participation, not just consultation, is challenging; people must feel that their input is valuable and may actually impact a decision. Planners must avoid relegating public participation to mere consultation, by instead establishing a dialogue with the public on the fate of a project; only then can a true democratic process be achieved (Forester, 1982). Indeed, Arnstein (1969:216) observed that "participation without redistribution of power is an empty and frustrating process for the powerless". Unfortunately, HIA has also experienced the same participation pitfalls as those of EA: one researcher, documenting an HIA for a housing regeneration project in the UK, found that stakeholders felt public meetings to be "plentiful, not meaningful" (Kearney, 2004:224). Ideally, HIA should facilitate a dialogue between health care and EA practitioners in order to address public concerns and create a transparent process (BMA, 1999). These individuals have been shown to garner considerable public trust and could serve to enhance public participation (Davies and Sadler, 1997). Finally, Quigley et al. (2006) submitted guiding principles of HIA to consider during assessments: sustainable development, equity, transparency, the health of future generations, as well as complying with an inclusive definition of health. These tenets of HIA overlap with several purposes of the CEAA, thus promoting the practice of integrated assessment. 


\subsubsection{Health Considerations in CEAA}

While some other jurisdictions preferred investigating health impacts of a project, policy or program through an HIA independent of the EA process, the Canadian approach was to minimize duplication of efforts and integrate the two assessment techniques (Eyles, 1999). However, several barriers hindered such inclusion: little administrative incentive, poor agreement on both the scope of issues and procedures to be used, as well as insufficient guidance material on the process (Davies and Sadler, 1997). To help improve the situation, a Task Force on HIA was assembled in 1992 by the Federal/Provincial/Territorial Committee on Environmental and Occupational Health, whose purpose was to improve the knowledge base of HIA. During the six regional workshops held by the Task Force, several principles were recognized: HIA is an essential component of EA, public participation must be emphasized throughout all areas of $\mathrm{HIA}$ and acceptance of the WHO definition of health is necessary (Health Canada, 2004a). Concerns were focused on the need for robust indicators and methods, with the importance of baseline community health information being particularly stressed (Spiegel and Yassi, 1997). The results of these workshops produced four extensive volumes of guidance on the inclusion of HIA in the Canadian EA process; however, no formal changes to CEAA were mandated (Health Canada, $2004 a, b, c, d)$.

Despite the work of the HIA Task Force, the definition of "environment" in CEAA excludes specific mention of human health and instead concentrates on the biophysical environment (CEAA, s. 2(1a-c)). Alternatively, considerations to "health and socioeconomic conditions" are provided in the definition of an "environmental effect" (CEAA, 
s. 2). Noble and Storey (2005) asserted this subtlety implies effects on human health will only be considered when they result from project-induced changes to the environment, and not when they are direct consequences of the project itself. Therefore, certain social effects, independent of changes to the biophysical environment, will be ignored. Omitting any adverse human impacts of this nature would be contrary to the promotion of sustainable development, one of the purposes of CEAA (CEAA, s. 4(1b)). Furthermore, although it is encouraged, there is no formal requirement in CEAA for proponents to conduct a separate HIA or HRA. Canada has also been criticized for mainly assessing biomedical aspects of health, as opposed to including the broad spectrum of determinants, called for in the WHO definition (Cole and Fielding, 2007). Finally, the protection of human health may not be a top priority for certain RAs; for example, the Ministry of Industry and Finance Canada may downplay adverse impacts during project approval (Herring, 2005). For these reasons, it is necessary to examine the rigor and value of efforts to include health impacts in the Canadian EA process.

\subsubsection{The Need for Evaluation}

Undoubtedly influenced by the increasing production of HIA guidance material, EA practitioners are now more inclined to include potential human health impacts within EISs. HIA research initiatives now have a more broad, yet demanding task: Has HIA helped catalyze a shift in the nature of development to improve public health and reduce inequality (Kemm et al., 2004)? 
A number of researchers have created evaluation criteria to gauge the quality of HIAs for different projects (Quigley and Taylor, 2004; Bekker et al., 2005; Parry and Kemm, 2005; Ali et al., 2009). Three broad evaluation categories emerged upon reviewing these frameworks: (1) "prediction" criteria, examining the methods used to predict potential health impacts; (2) "process" criteria, including the timeliness of the assessment and the degree to which stakeholders were involved; and (3) "decisionmaking" criteria, scrutinizing the use of HIA recommendations by decison-makers. Table 2.2 highlights overlapping criteria found in these HIA evaluation frameworks.

\section{Prediction}

- Clear definition of 'health' given

- Used best available evidence

- Established baseline health of affected communities

- Compared alternatives

- Clear description of data collection and analysis methods provided

- Health determinants justified

- All possible cause/effect pathways examined

- Positive and negative impacts given

- Uncertainties discussed

- Monitoring responsibilities established

- Findings easy to understand/read

\section{Process}

- Stakeholders identified and involved throughout the process

- Started at appropriate time

- Recommendations given to decision-makers at appropriate time

- Project information made available to stakeholders

- Post-HIA responsibilities (e.g. monitoring) detailed

\section{Decision-making}

- Evidence of HIA recommendations implemented

- Transparency in decision-making

- No evidence of bias

Table 2.2. HIA evaluation criteria (collated from Quigley and Taylor, 2004; Bekker et al., 2005; Parry and Kemm, 2005; Ali et al., 2009). 
Other authors have advocated the integration of HRA into the EA process as a valuable method to improve and evaluate the consideration of project health risks (Harvey, 1990; Canter, 1993; Kwiatkowski, 1999; Demidova and Cherp, 2005). A review of these guidance materials revealed a number of trends. Although the process of HRA is not outlined until subsection 2.3.3.1.2, it is of value to the current discussion to identify parallels between the processes of HIA and HRA. Table 2.3 displays recommendations on how to effectively integrate HRA into an EA, and does so via the four standard steps of an HRA.

\begin{tabular}{l} 
Hazard Identification \\
\hline - Project description given with potential health hazards \\
- Outlined different phases of project development \\
- Technological description of project provided \\
- Harmful materials on site listed \\
- Evidence of public input
\end{tabular}

\section{Exposure Assessment}

- Baselines of existing environmental contamination established

- Characterized health status of local population

- Described different exposure pathways

- Employed environmental monitoring/modeling

- Considered environmental fate

- Assessed both normal and accidental conditions

\section{Dose-response Assessment}

- Assembled toxicity information on relevant agents

- Impacts given as magnitude of health effects in exposed population

\section{Risk Characterization}

- Quantitative estimation of risks given

- Compared risks with regulatory limits

- Considered both consequences and probabilities

- Addressed project health implications in proposed mitigation/monitoring

- Compared risks of project alternatives

- Acknowledged and discussed uncertainties and confidence limits

- Presented information for non-specialists

- Addressed public perception of risk

Table 2.3. Criteria to evaluate HRA integration in EA (collated from Harvey, 1990; Canter, 1993; Kwiatkowski, 1998; Demidova and Cherp, 2005). 
By comparing Tables 2.2 and 2.3, it is apparent that high quality HIAs and HRAs would ensure public participation throughout the process, provide findings in an easily understandable manner, establish baseline health statuses of exposed populations, examine all relevant exposure pathways, explicitly address uncertainty, and define postdecision monitoring/mitigation responsibilities.

Despite extensive efforts promoting the use of HIA, research suggests that more evidence on the value of HIA and EA integration is still required: previous studies in the UK have shown that nearly $90-95 \%$ of EAs have excluded health to any substantial depth (Davies and Sadler, 1997); a recent analysis of EAs in Australia found that the health sector was not being consulted during the assessment process (Harris et al., 2009); and a review of 49 EISs in the US found more than half failed to provide an examination of health impacts (Steinemann, 2000). Although the mandate of NEPA is to "stimulate the health and welfare of man", the US has been quite reluctant to address human health in EA; the first comprehensive HIA completed within an EA was only recently documented by Wernham (2007). Nevertheless, researchers in that jurisdiction have called for making HIA more prevalent through the use of the existing NEPA (EA) legislation (Bhatia and Wernham, 2008).

An investigation into health impacts in Canadian EISs funded by the Canadian Environmental Assessment Agency found that human impact considerations were only evident in comprehensive studies and panel reviews (Yap, 2003). Information provided in screening reports was determined to be insufficient for analysis. This fact is alarming since over $99 \%$ of EAs under CEAA are completed at the screening level, which truncates opportunities for public participation (Herring, 2005). This study concluded by 
encouraging RAs to involve local stakeholders in the scoping phase of screenings, more typical in comprehensive studies and panel reviews, by circulating the Terms of Reference for public comment. This practice would provide the public with an opportunity to influence situations where human health impacts are given insufficient consideration.

From these EA reviews, it is clear that much more research, particularly evaluation, is required to improve the state of integrating HIA into EA. The following subsections detail the relevant health issues surrounding nuclear power, further validating the need to evaluate EISs pertaining to that sector.

\subsection{Nuclear Power}

\subsubsection{The Canadian Context}

The unprecedented research efforts put forth in the Manhattan Project during the Second World War enabled the possibility of using nuclear fission as an energy source. Shortly after the conclusion of the war, Canada enacted the Atomic Energy Control Act in 1946, thus giving the Atomic Energy Control Board (AECB) the responsibility to federally regulate all facilities pertaining to nuclear power. In 1952, the Government of Canada established a crown corporation, Atomic Energy of Canada Limited (AECL), which went on to design the CANDU (CANada Deuterium Uranium) reactor. The timing of the CANDU reactor as a potential energy source in Canada was ideal: undeveloped hydraulic capacity was becoming scarce and the price of coal was on the rise (Brooks, 1993). 
The atom of the uranium isotope $\mathrm{U}-235$ is naturally radioactive; its nucleus can spontaneously eject a neutron, coinciding with a release of kinetic energy. Nuclear fission is the process by which one such neutron enters another $\mathrm{U}-235$ atom to cause a similar neutron ejection, eventually forming a chain reaction. A reaction of this nature can be sustained in a nuclear reactor to produce electricity, only if the following conditions are satisfied: using a fissionable material that has a high probability of capturing neutrons, controlling the amount of nuclear fission by preventing runaway reactions, and removing the generated heat (Fullwood, 2000).

The design of the CANDU reactor is unique: contrary to US light water reactors, it employs natural uranium ${ }^{12}$ as the fuel source and heavy water as both the moderator and coolant. At the time of development of this technology, Canada had abundant uranium resources, but lacked the enrichment technology that the US had devised during the Manhattan Project ${ }^{13}$; therefore, it was necessary to create a reactor that could readily utilize a lower concentration of U-235. Heavy water ${ }^{14}$ was selected as the moderator, which slows down ejected neutrons and promotes a sustained chain reaction (Brooks, 1993).

\footnotetext{
12 The term "natural uranium" can be deceiving, as it is not synonymous with the uranium found in nature. The uranium found in nature must still to be refined and concentrated into a usable form, as do all other metals. It is referred to as natural uranium, because it maintains the same isotopic ratio as the uranium found in nature (see next footnote).

13 Natural uranium consists predominantly of the isotope U-238, with only $0.7 \%$ of U-235. Enriching uranium is the process by which the amount of U-235 is increased to concentrations of approximately $2-3 \%$. Similarly, depleted uranium means that the concentration of U-235 has been reduced to levels below the natural concentration of $0.7 \%$ (Gronlund et al., 2007).

14 Heavy water is composed of deuterium oxide, which is essentially a water molecule, except that one of the hydrogen atoms has been replaced with a deuterium atom. Deuterium is an isotope of hydrogen that has a nucleus of one proton and one neutron (Fullwood, 2000).
} 
With the successful development of the CANDU reactor, four commercial reactors at the Pickering A site came online between 1971 and 1973. Four more reactors were then built at the Bruce A site from 1977-79. Throughout the 1980s, additional reactors were added: Pickering B and Bruce B (Brooks, 1993). Finally four reactors were constructed at a new site, Darlington, which came online between 1990 and 1993. Although the environmental community had urged for the Darlington project to undergo an EA, the Premier of Ontario, at the time, claimed that the immediate need for electricity production precluded the possibility of conducting a lengthy assessment and thus exempted the project (Janisch, 1996). The development of all of these nuclear power plants preceded the legal mandate for EA under $C E A A$, and as such, no federal EAs were conducted prior to reactor construction (Health Canada, 2004b).

The 1990s witnessed events that significantly eroded the public's trust in Ontario's nuclear industry. Firstly, a series of epidemiological studies were completed on populations surrounding the Bruce and Pickering nuclear stations, raising concerns over possible increased incidences of leukemia and birth defects ${ }^{15}$ (Fairlie, 2007). Secondly, two years after the promulgation of CEAA, the president of Ontario Hydro ${ }^{16}$ $(\mathrm{OH}$, the operator of the Pickering and Darlington plants, though now Ontario Power Generation, OPG ${ }^{17}$ ) was forced to order an Independent Integrated Performance Assessment (IIPA) of Ontario's nuclear power plants, some of which had experienced

\footnotetext{
15 Most of these studies were ecological studies, meaning they examined data at the population level, not the individual; such studies carry low statistical power. Accordingly, these Ontario studies demonstrated mixed results, which were challenging to interpret (Fairlie, 2007).

$16 \mathrm{OH}$, an electric utility monopoly in Ontario, had its assets split into five entities in 1999, one of which was to assume the generation business: OPG (OPG, 2000a).

17 Both $\mathrm{OH}$ and OPG are used, depending on the context.
} 
notable operational mishaps in recent years ${ }^{18}$. The IIPA resulted in a scathing report of $\mathrm{OH}$, giving each reactor a grade of "minimally acceptable" and found evidence of "alcohol and drug use by employees on the job, contradictory and counter-productive management structures, major communication gaps, and a lack of leadership at all levels of the nuclear division" (Arai, 2001:418). Due to the urgency of the identified problems, $\mathrm{OH}$ decided to temporarily shut down the four units at Pickering $\mathrm{A}$ and three units at Bruce $A$ to better direct resources. The shutdown of these reactors, albeit temporary, constituted the single largest voluntary reduction in nuclear generating capacity (Arai, 2001). CEAA was then triggered for the subsequent return to service of the Pickering A units ${ }^{19}$; as well as for the refurbishment of Pickering B in 2007 (CNSC, 2009a).

At the time of the IIPA, OH was the operator for all of Ontario's nuclear plants; however, a deal was finalized in May 2001 whereby Bruce Power20 (BP) would lease all eight of the Bruce reactors from OPG (Blake, 2005). Following this transaction, BP promptly initiated the approvals process for the laid-up reactors at Bruce A, which again triggered CEAA for reactor restart and refurbishment (CNSC, 2009a).

During the various EA proceedings for reactor life extensions at the Bruce and Darlington stations, the government of Ontario released the Supply Mix Directive in 2006 to highlight its goals for Ontario's future energy needs. To realize these

\footnotetext{
18 Nuclear accidents, including several at the Ontario plants, are examined in 2.3.3.2.1.

${ }^{19}$ Although CEAA ensured a legal obligation to conduct an EA, OPG attempted to evade such scrutiny by arguing, unsuccessfully, that the return to service project fell under CEAA's Exclusion List (The Standing Senate Committee on Energy, The Environment and Natural Resources, 2001).

20 Bruce Power represents a partnership between Cameco Corporation, TransCanada Corporation and BPC Generation Infrastructure Trust (Blake, 2005).
} 
requirements, the Ontario Power Authority (OPA) produced the Integrated Power System Plan (IPSP) for the period 2008-2027 to achieve a combination of implementing customer conservation measures and acquiring new supply resources. The main objectives of the Directive, which the IPSP serves to accomplish in a cost-effective manner, include maximizing contributions of renewable sources, replacing coal-fired facilities by 2014 and most significant to the present discussion, fulfilling baseload requirements with nuclear power (OPA, 2008). To that end, the construction of new reactors $^{21}$ has been proposed at both the Bruce and Darlington sites, thus initiating the EA process (CNSC, 2010). These proposals are unique when taking into consideration that no nuclear power plants have been ordered in North America since 1978 (Bodansky, 2004); the existence of CEAA now requires the completion of an EA prior to reactor construction.

\subsubsection{The CNSC}

Although the Atomic Energy Control Act created the AECB in 1946 to regulate nuclear activities in Canada, the Nuclear Safety and Control Act (NSCA) of $1997^{22}$ gave rise to its regulatory successor: the CNSC (NSCA, 1997). The NSCA was necessary in order to strengthen the regulation of Canada's nuclear industry, as the objectivity of the AECB had been increasingly questioned. Oxman et al. (1989:51) expressed concern

\footnotetext{
21 Giving serious consideration to building new reactors is not unique to Ontario: Finan et al. (2006) concluded that using nuclear power to provide energy at oil sands facilities in Alberta would be "feasible. practical and economical" and the Government of Saskatchewan commissioned a report to examine the potential use of nuclear power in that province, which subsequently initiated an investigation into possible public health implications (Lemstra, 2009).

22 Note that the timing of this act, which revamps the nuclear regulatory system in Canada, coincides with the highly negative findings of the IIPA.
} 
over this matter by stating that "the relationship between the AECB and Ontario Hydro is not ideal. It depends heavily on informal discussion and exchange of correspondence". The CNSC was assigned to be wholly responsible for regulating all activities pertaining to nuclear power and also to "prevent unreasonable risk, to the environment and to the health and safety of persons, associated with that development, production, possession or use" (NSCA, 1997: s. 9(a)(i)). This designation made the CNSC the RA23 for EAs of all nuclear power related projects. Additionally, part of the CNSC mandate is to ensure that Canadian facilities adhere to international best practices in nuclear safety (CNSC, 2008b).

Despite these commitments, the ability to protect the safety of Canadians from nuclear hazards has been previously compromised by encroaching political interests. For example, the president of the CNSC, Linda Keen, was fired for shutting down the NRU reactor at Chalk River in late 2007. That reactor is responsible for producing a significant proportion of the global radioisotopes for medical use. The CNSC had made certain recommendations for improvements, which AECL had not honoured (Thompson, 2008c). It was calculated that there was a one in a thousand chance of an accident occurring at this reactor; this risk is a thousand times greater than the international standard for an acceptable risk of one in a million. However, the need to produce medical isotopes for a global market overshadowed public safety: Parliament voted to override her decision, restarting the reactor and terminating her position as president

\footnotetext{
${ }^{23}$ Recall that the duties of an RA include providing guidance on the necessary environmental effects to be investigated in an EA and deciding whether to grant final project approval (Herring, 2005).
} 
(Risk of restarting nuclear reactor too high: Keen, 2008). Ultimately the NRU reactor was shutdown in May of 2009, following a series of tritium²4 leaks (Buckthought, 2009).

Events such as these illustrate that even a supposedly independent regulatory body, such as the CNSC, cannot be completely free from political influence, even if it is at the cost of risking human health. In the case of the new reactor projects, political interests are two-fold: the need to fill the energy gap presented by the inevitable closing of coal-fired plants in Ontario by 2014 and the prospect of a Canadian company (AECL) providing the technology for the new reactors. Mounting political pressure to ensure project approval could very well jeopardize the CNSC's primary responsibility to protect public health and safety; as Birley (2003:317) observed, "governments may circumvent their own regulations when expedient". For these reasons, it is necessary to examine the inclusion of health impacts in initiated nuclear power EAs, as prescribed by the CNSC.

\subsubsection{Health Impacts of Nuclear Power}

The following section documents the human health impacts from nuclear power plants. First, the discussion focuses on health risks 25 from chronic radioactive exposure during normal operation. The process of HRA is then described; this is the current method used to ensure exposure levels are kept within regulatory limits. To conclude this chapter, a brief history of nuclear accidents is given, as well as a discussion of the

\footnotetext{
24 Tritium is a radioactive isotope of hydrogen and is discussed in detail in subsection 2.3.3.1.1.

${ }^{25} \mathrm{~A}$ health risk is the probability that a hazard, of some calculated magnitude, will harm a particular group of people; whereas a health impact is a change in health risk, attributed to a project (Demidova and Cherp, 2005).
} 
regulatory tool, PRA. This section concludes with an examination of the possible acute health risks from large-scale releases of radiation.

\subsubsection{Chronic Health Impacts}

\subsection{Background on lonizing Radiation}

lonizing radiation, as opposed to non-ionizing radiation, is referred to as such because it carries enough energy to eject electrons from exposed atoms ${ }^{26}$, thus altering their molecular charge (Walker, 2000). Exposure to natural sources of ionizing radiation include cosmic rays and radioactive elements in the Earth's crust (Makhijani et al., 2006). Anthropogenic sources of radiation are created by medical and nuclear power industries, among others. The fission of uranium fuel in a nuclear reactor is responsible for creating more than 1500 known radionuclides, many of which do not exist in nature (Walker, 2000; Rahn and Upton, 2007). These radionuclides are routinely emitted in small levels during normal operations; the potential for large releases exists through the possibility of nuclear accidents (see subsection 2.3.3.2.1).

Radionuclides are essentially atoms with unstable nuclei, having the ability to undergo spontaneous decay. This type of disintegration releases different forms of radiation: alpha, beta, gamma or neutron ${ }^{27}$. Additionally, these radionuclides have halflives 28 ranging from a fraction of a second to hundreds of thousands of years, ultimately

\footnotetext{
${ }^{26}$ Radiation with energy higher than 12.5 electron volts per photon is considered ionizing (Rahn and Upton, 2007).

27 Alpha radiation represents a helium nucleus; beta radiation entails a low energy electron and gamma radiation involves a high-energy photon (CERRIE, 2004).

${ }^{28} \mathrm{~A}$ half-life is "the time taken for the radioactivity of a particular radionuclide to decay to half of its initial value" (CERRIE, 2004:6).
} 
giving rise to new radioisotopes until a stable atom is rendered ${ }^{29}$. Each of these subsequent disintegrations produces a decay particle, with energies ranging from tens to millions of electron volts (Fairlie, 2005).

To further complicate matters, fundamental differences exist between external and internal exposures to radiation. Gamma rays and neutrons represent external sources of radiation, since they are able to penetrate the body and deposit their energies within various tissues and organs. Alpha and beta emitters, whose energies are weakly penetrating, must be ingested or inhaled before causing physiological insult. If such internal exposure occurs, the radioactive particle may be either excreted from the body or embedded in a bodily tissue; the latter represents the greater health threat. If a radioactive particle is deposited somewhere in the body, its specific half-life ${ }^{30}$ then dictates the amount of energy absorbed in surrounding tissues. Furthermore, many radionuclides, upon entry to the body, show a preference for specific body parts ${ }^{31}$, thus presenting distinct health risks (CERRIE, 2004). The important point to understand here is that there are many different sources and types of radiation, all of which need to be accounted before quantifying the health risks ${ }^{32}$.

It has been extensively documented that high doses of ionizing radiation can lead to cancer, as demonstrated by atomic bomb survivors (Sont et al., 2001). It is less

\footnotetext{
29 For instance, the isotope U-238 eventually decays until $\mathrm{Pb}-206$ is produced. The numerous intermediate isotopes undergo various alpha and beta decays before a stable atom is finally achieved (Rahn and Upton, 2007).

30 In addition to the radioactive half-life, the biological half-life of internally deposited radioisotopes is of interest: the average time that a specific radionuclide resides in the body (Makhijani et al., 2006).

${ }^{31}$ For instance, the isotope $\mathrm{I}-131$ gathers at the thyroid and Pu-239 tends to gravitate toward bone surfaces (CERRIE, 2004).

32 The next subsection further addresses this issue.
} 
clear, however, for researchers to determine the carcinogenic potential of chronic exposure to low doses. Although one track of ionizing radiation through the nucleus of a cell is theoretically sufficient to induce cancer (Nussbaum, 1998), epidemiological problems with studying small exposed populations have been documented by many (Sont et al., 2001; Brenner et al., 2003; Muirhead, 2008). In fact, one researcher commented on the sensitivity of epidemiological studies in general: "a public health catastrophe is a health effect so powerful that even an epidemiological study can detect it” (Ozonoff, 1994:504). Alternatively, large studies, involving much higher statistical power, use data from nuclear workers around the globe to research the effects of protracted exposure to low levels of ionizing radiation. A significant association has been demonstrated between increasing radiation dose and the risk of mortality from all cancers $^{33}$ (Cardis et al., 2007). In Canada, Sont et al. (2001) investigated cancer incidence from occupational radiation exposures using the National Dose Registry of Canada. Similar to the results of Cardis et al. (2007), several notable associations were found: increases in all cancers combined for males and females, and increases in colon, pancreas, testis and thyroid cancers for males. Many studies have also shown that close proximity to nuclear facilities can increase the incidence of childhood leukemia, although this remains a controversial finding in the epidemiological community for various reasons $^{34}$ (Baker and Hoel, 2007; Fairlie, 2010). Finally, research is

\footnotetext{
${ }^{33}$ Canadian workers experienced the highest elevated risks with increasing exposures; the reason for this trend was not known.

34 The main reason that ionizing radiation has been rejected as a cause for increased childhood leukemia incidences is because exposure levels are considered far too low to cause such an effect. A popular alternative hypothesis is that an infectious agent is responsible for the increase, exacerbated by population mixing. As tempting as this theory may be, no such agent has yet been identified (Baker and Hoel, 2007).
} 
accumulating on the increased incidence of cardiovascular disease and stroke from prolonged exposure to ionizing radiation ${ }^{35}$ (Richardson, 2009; Shimizu et al., 2010).

Before discussing HRA, a brief note on tritium is necessary. Tritium is a radioactive isotope of hydrogen possessing two neutrons, having a half-life of 12.3 years and releasing beta radiation upon disintegration (Fairlie, 2007). This isotope is formed once in every 10000 fissions of U-235 and also when a deuteron, the isotope of hydrogen in heavy water, captures a neutron. CANDU reactors use massive volumes of heavy water, which is responsible for producing much more tritium than other reactor designs (Little and Wakeford, 2008). Tritium has several properties that make it a unique human health hazard: extreme environmental mobility, a long biological half-life (Fairlie, 2008) and the ability to incorporate into DNA (HPA, 2007). Public concern over tritium was amplified in the 1990s when the Ontario drinking water standard was set to $7000 \mathrm{~Bq} 36 / \mathrm{L}$, a value 70 times greater than that of the EU (McMullan and Eyles, 1999). More unnerving, however, is the fact that although tritium is one of the more prevalent radionuclides in radiation protection (Fairlie, 2008), a recent review questioned the paucity of epidemiological studies on the health effects of tritium exposure (Little and Wakeford, 2008).

\subsection{Health Risk Assessment}

HRA is a process whereby the magnitude and probability of adverse health effects from chemicals and radiological agents are quantified, usually in terms of excess

\footnotetext{
35 It takes a great deal of research to establish causality by way of epidemiological studies. Most notably, Sir Bradford Hill put forth tests of causality in 1965 to help determine the merit of a suspected association, including such parameters as strength, consistency, specificity, and plausibility (Hill, 1965).

${ }^{36}$ The Becquerel $(\mathrm{Bq})$ is a measurement of the amount of radioactivity: $1 \mathrm{~Bq}=1$ disintegration per second.
} 
deaths from cancer (Campbell-Mohn and Applegate, 1999). The first HRA methodology was proposed by Lowrance (1975), then subsequently revised by the National Academy of Sciences (NAS) in 1983 with the release of Risk Assessment in the Federal Government: Managing the Process (also known as the "Red Book") (Doull, 2003). Four main steps of HRA were defined, as outlined in Table 2.4. A brief explanation of the pertinent features of the radiological risk assessment process will follow.

\begin{tabular}{|c|c|}
\hline HRA Step & Description \\
\hline Hazard Identification & $\begin{array}{l}\text {-What are the possible toxins? } \\
\text { - Are these toxins relevant to humans? }\end{array}$ \\
\hline Exposure Assessment & $\begin{array}{l}\text {-What are the important exposure routes } \\
\text { to humans? } \\
\text {-What doses do humans receive? }\end{array}$ \\
\hline Dose-response Assessment & $\begin{array}{l}\text {-What is the nature of the relationship } \\
\text { between dose and response? } \\
\text { - What is the quantitative relationship } \\
\text { between dose and probability of toxic } \\
\text { response at levels of human exposure? }\end{array}$ \\
\hline Risk Characterization & $\begin{array}{l}\text { What is the probability of toxicity? } \\
\text { - What are the uncertainties inherent in the } \\
\text { calculations? }\end{array}$ \\
\hline
\end{tabular}

Table 2.4. The fundamental steps of the HRA process (adapted from Jardine et al., 2003).

The hazard identification step, with regard to nuclear reactors, refers to generating an accurate definition of the source term ${ }^{37}$. An insufficient hazard identification would seriously undermine the credibility of any subsequent calculations (Till, 2008). Exposure assessment is a multidisciplinary process, dominated by

\footnotetext{
37 The expression, "source term", "refers to the quantities and compositions of the radioactive materials released, locations of the release points, and the rates of release during the times considered in the assessment" (Voillequé, 2008:31).
} 
modeling the atmospheric dispersion of radionuclides. Combining rates of inhalation and ingestion with levels of environmental radioactivity, a general estimate of an exposed individual's dose can be determined (Paustenbach, 2000). The dose-response curve for ionizing radiation is a subject of perpetual disagreement. Shrader-Frechette (1993) observes three conflicting viewpoints on this issue: environmentalists claim a supralinear curve, highlighting the dangers of low doses; industry endorses a linear model, but with a threshold from which there are no effects below a certain level; and health physics associations maintain a linear no threshold (LNT) approach, whereby an increased dose implies a commensurate risk of developing cancer. Some others even advocate a hormetic approach, claiming that low levels of radiation present health benefits ${ }^{38}$; although most researchers find this theory to be untenable (NAS, 2006). Nevertheless, the International Committee on Radiological Protection (ICRP ${ }^{39}$ ) contends that not enough compelling evidence exists to render the LNT void. Finally, the risk characterization step quantifies the increased risk of cancer deaths from the calculated exposure levels, and provides an uncertainty analysis (Jardine et al., 2003).

The two most common criticisms of this tool state that HRAs are "merely exercises in decision justification" (Ozonoff, 1993:503) and that the assumptions are laden with value judgments (Tickner, 2007). In few other contexts is the second point

\footnotetext{
${ }^{38}$ The contemporary belief of radiation hormesis is not unique. Shortly after the discovery of ionizing radiation, the consumption of radium water in the early 20th century was thought to provide health benefits: "for the first few months after taking radium into the body there is a sensation of well-being and general physical improvement. Soon, however, the deadly alpha ray bombardment of the blood producing centers begins to be felt, and death follows in a year or more, depending on the total quantity of radium fixed in the system" (Evans, 1933:1019). Though current advocates of radiation hormesis support their health claims with empirical evidence, the parallel is still interesting.

39 The ICRP is an international group of scientists that studies and disseminates information on radiological risks to governments around the globe.
} 
more applicable than in radiological risk assessment. For instance, as detailed above, a host of weighting factors are applied to accommodate for the different types of radiation ${ }^{40}$. All of this information is then combined to devise Dose Conversion Factors (DCFs) for each radionuclide; DCFs are used to transform inhaled/ingested radioactivity (measured in $\mathrm{Bq}$ ) into measurements of the effective dose (measured in Sieverts (Sv)) (Hamby, 1999). The radioactive doses resulting from all exposure pathways are then summed to ensure the total is below $1 \mathrm{mSv}$, the accepted maximum annual exposure for the public (ICRP, 2006). Although the ICRP constantly updates its risk factors, there is constant disagreement over the correct value of weighting factors, most notably for internal emitters (Goodhead, 2009).

Despite these obvious imperfections, HRA remains the best tool for regulatory agencies to ensure the public is protected from an unreasonable level of risk (Callahan and Sexton, 2007). Problems arise, however, when these shortcomings are not acknowledged and uncertainties are ignored; thus, transparency and justification throughout the entire process is essential.

\subsubsection{Acute Health Impacts}

\subsection{Nuclear Accidents}

Commercial nuclear reactors exploit the natural radioactive property of uranium to produce electricity. The success of this relies on attaining a sustained nuclear fission chain reaction among the nuclei of U-235 atoms. Achieving this is a delicate balancing

\footnotetext{
40 Relative Biological Effectiveness factors in the effectiveness of different types of radiation to cause damage, tissue weighting factors consider the vulnerabilities of various organs to the effects of ionizing radiation, and even dose rate effectiveness accounts for the time in which the dose is administered (Rahn and Upton, 2007).
} 
act: a reaction must occur to generate enough heat, but too much and it could experience an excessive power surge, spiraling out of control. This undesirable situation is known as a criticality accident, which could potentially lead to a core meltdown. However, numerous safety apparatuses do exist to limit the possibility of this happening; for example, control rods are dropped between fuel rods to act as "neutron sponges" in order to stop fission reactions (Fullwood, 2000).

The most prominent nuclear accident in the US, involving a reactor core, took place at the Three Mile Island (TMI) plant in Pennsylvania. Early on March 28, 1979, a sequence of events caused one of the units to experience a loss of coolant accident $\left(\mathrm{LOCA}^{41}\right)$. Radiation monitors in the plant exceeded their measuring capacity within hours. The growth of a hydrogen bubble prompted fears over the possibility of a hydrogen explosion, as days passed before the reactor could be successfully shut down. Delays and imperfections in the set up of radiation monitors external to the plant have created much controversy over the extent of health effects to the surrounding population. Despite official statements of the impossibility of significant radiation exposures, hundreds of local residents complained of symptoms aligned with acute radiation sickness ${ }^{42}$ (Wing, 2003). Re-evaluations of data used in official reports stating that no observable health effects occurred have demonstrated significant positive associations between accident dose and cancer incidence, especially those of leukemia (Wing et al., 1997). The official causes of the accident have been attributed to a

\footnotetext{
${ }^{41}$ A LOCA can lead to voids in the coolant water. These voids can cause one of two situations to occur, depending on the design of the reactor: (1) a positive void, meaning that the reactor could experience a surge in power levels or (2) a negative void, indicating that the reactor would undergo a reduction of power (Thompson, 2000).

42 Such symptoms include erythema, vomiting and hair loss.
} 
number of factors, including mechanical failure, operator error, plant design flaws and regulatory incompetence (Mosey, 2006). Perrow (1999) has further argued that the complicated designs of nuclear power plants create "normal accidents": outcomes that cannot always be prevented, due to the unpredictability of the many tightly-coupled components on which these systems rely. Moreover, upper management in plants may increase the likelihood of such accidents occurring by the willful neglect of maintenance and safety rules ${ }^{43}$ (Perrow, 2007).

The core meltdown at the Chernobyl nuclear plant on April 26, 1986 was undoubtedly the worst nuclear accident thus far. At the time of the accident, the emergency core cooling system had been shut off in order to test a safety procedure. The reactor ${ }^{44}$ soon became overheated and caused two explosions, releasing vast amounts of radioactivity into the surrounding environment. Instead of alerting the world of the disaster, the Soviet government provided misinformation about the incident; it was not until April 28 that radiation detectors at a Swedish nuclear plant indicated that an accidental release had occurred (Rahu, 2003).

The extent of the resulting environmental and health effects of Chernobyl have been subject to intense debate. Four months after the accident, the Director General of the International Atomic Energy Agency (IAEA), Hans Blix, declared that "the world could tolerate a nuclear accident as serious as Chernobyl every year" (Mittica, 2006:17). The most widely accepted health detriment from this disaster is an increase in childhood thyroid cancer in individuals living in the affected regions of Belarus, Russia and

\footnotetext{
43 The IIPA's findings of widespread management problems among Ontario's nuclear industry appear even more disturbing in light of Perrow's insights.

44 The reactors at Chernobyl were Soviet-design RBMK reactors, which share a common characteristic with CANDU reactors: a positive void coefficient (Thompson, 2008a)
} 
Ukraine (NAS, 2006). However, many researchers have also documented increases in solid cancers other than thyroid tumors, including leukemia, Down's syndrome, and stillbirths (Noschenko et al., 2002; Fairlie and Sumner, 2006; Mittica, 2006; Nussbaum, 2007; Busby et al., 2009). The reasons offered for such epidemiological chaos are many: poor health records in former Soviet Union nations (Rahu, 2003), worse, some medical examiners were forbidden to attribute health detriments to radiation (Shlyakhter and Wilson, 1992); many studies follow an ecological design and therefore, entail weak statistical power (Rahu, 2003); disagreement over the amount of radiation released and subsequent dispersion patterns (Nussbaum, 2007); and it has even been suggested that the incidence of early fetal death in high dose areas has biased cancer data by removing potential cases, when compared to lower dose regions (Busby et al., 2009). Nevertheless, continued research on the effects of Chernobyl may reveal further local and possibly even far reaching health consequences, since radiation was dispersed globally and cancer latencies can extend many decades (Mangano, 2009).

Accidents, such as LOCAs, have also occurred at Canadian nuclear power plants, albeit with much less serious outcomes than the previously described accident. For instance, Unit 2 at the Pickering A plant experienced such an event, which led to two fuel bundles being damaged. Fortunately, radiation was contained; however, Units 1-4 were shut down and subject to pressure tube replacement between 1989 and 1993 . Additionally, another LOCA occurred at the same Pickering unit, causing a 270 degree crack in one of the release valves and resulting in the release of 140 tonnes of heavy water into Lake Ontario (Mosey, 2006; Fairlie, 2007). Not to distinguish the Pickering facility, other reactors have also experienced similar operational mishaps: Bruce 
reactors leaked 12 and 25 tonnes of radioactive heavy water in 1990 and 1995, respectively ${ }^{45}$ (Fairlie, 2007).

\subsection{Probabilistic Risk Assessment}

Prior to the landmark Reactor Safety Study (RSS) in 1975, the nuclear industry divided nuclear accidents into two broad categories: design-basis, which were of limited severity, and beyond design-basis, which involved serious damage to the reactor core; this second type of accident was thought not to be possible (Thompson, 2008c). This was only until the Norman Rasmussen-led team of researchers conducted the RSS to answer an increasingly important question: Were nuclear plants safe? To accomplish this task they utilized a revolutionary technique known as PRA46; similar accident scenarios were grouped together and the probabilities of occurrence were assessed. This methodology is known as event tree analysis and would become a staple of all subsequent PRAs (Keller and Modarres, 2005). The results of the RSS demonstrated, contrary to previous beliefs, that the dominant risk to the public was determined to be core melting accidents; containment designs would ultimately fail during such scenarios ${ }^{47}$. Despite the value of the RSS, subsequent reviews have criticized some aspects of the study, including the peer review process and certain statistical analyses (Wall, 1980).

\footnotetext{
45 This is by no means an exhaustive list of accidents; see Fairlie (2007) for further reading.

46 Probabilistic Risk Assessment (PRA) is also referred to as Probabilistic Safety Assessment (PSA); the former term is more common and will thus be used.

${ }^{47}$ See Hayns (1999) for further conclusions of the RSS and a summary of other pioneering PRAs.
} 
The general model of carrying out a PRA follows a common sequence: develop and screen scenarios, model scenarios, estimate parameter ranges and uncertainties, perform calculations and finally, interpret the results (Garrick and Christie, 2002). In addition to these basic steps, there are three increasing levels of complexity in which the PRA may be completed (see Figure 2.1): Level 1 seeks to identify the probabilities of accident sequences leading to core damage via fault trees; Level 2 determines the potential nature of radioactive releases from a failure of containment during core damage scenarios; and Level 3 uses the release information derived in Level 2 to model the atmospheric dispersion of radionuclides, with the ultimate objective of predicting both health and socio-economic consequences (Fullwood, 2000; Thompson, 2008c).

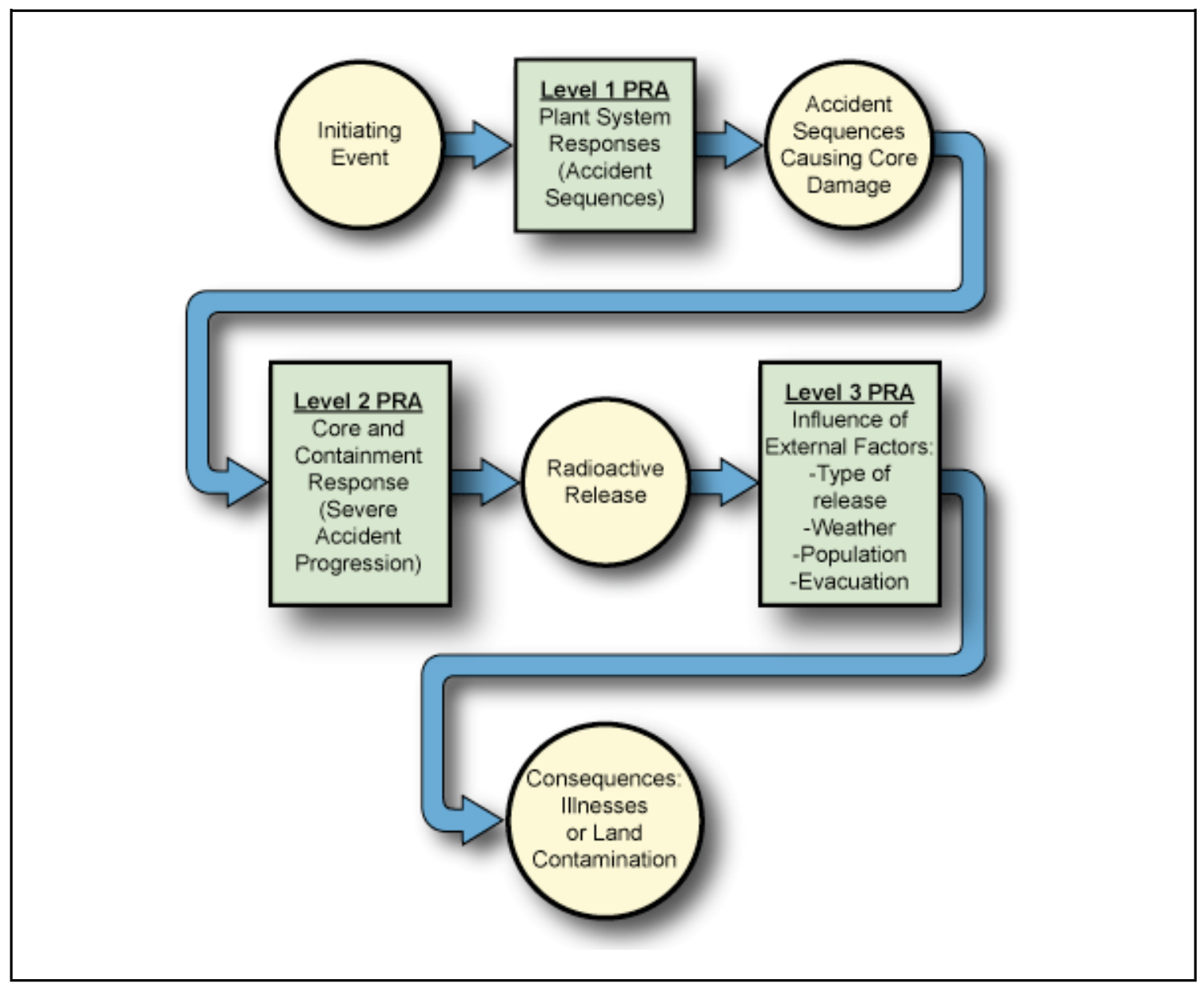

Figure 2.1. The three different levels of PRA (adapted from USNRC, 2007). 
Still considered to be the "state of the art" of PRA (Thompson, 2000), the USNRC produced a study known as NUREG-1150 in 1990 to examine severe risks presented by five different US nuclear plants (USNRC, 1990). NUREG-1150 constituted a Level 3 PRA and defined health through a number of metrics: early fatalities, total latent cancer fatalities and population dose within 50 miles. This study found that seemingly insignificant differences between plants could lead to accidents with large differences in the amount, type and probability of radioactive releases (Breeding et al., 1992). Additionally, NUREG-1150 considered initiating events that were both internal and external ${ }^{48}$ to derive accident sequences for each plant, with the conclusion that "external events are significant in the total safety profile of plants" (USNRC, 1990:8-15). To summarize, NUREG-1150 demonstrated that different nuclear plants, characterized by specific technologies and unique locations, are capable of producing very distinct health consequences.

The CNSC issued a regulatory document in 2005 to provide guidance on PRA, S-294; such guidance was needed, as the NSCA omitted any reference to PRA (CNSC, 2005b). This document states that a Level 2 PRA must be conducted before a license to construct or operate a nuclear power plant is granted. PRAs must also reflect the actual plant that is built and operated, and must be updated at least every three years. The S-294 does not explicitly address refurbishment projects; however, it is presumed that an updated PRA would be necessary before an operating license could be granted, in order to accurately reflect the changes proposed in plant refurbishment. Although the CNSC instructs the inclusion of both internal and external events, the licensee may

\footnotetext{
48 Internal events include such occurrences as human error and equipment failure, while external events encompass earthquakes and fires, among others (Thompson, 2008c).
} 
"choose an alternative analysis method to conduct the assessment" of external events (CNSC, 2005b:2).

\subsection{Summary}

This chapter began with an overview of the CEAA framework and the EA process in Canada. Efforts to include HIA into EA were then described, with a justification for the need to evaluate such efforts. The Canadian nuclear power industry was outlined before discussing a two-pronged approach for examining the health implications of operating nuclear power plants. Firstly, background information on ionizing radiation was provided prior to explaining HRA, a tool to regulate human exposure levels to ionizing radiation. Secondly, the acute health risks from higher exposures to ionizing radiation were investigated. Significant nuclear accidents were listed, with consequent health detriments detailed. This led into a discussion of PRA, a technique to determine the probability and magnitude of various nuclear accident scenarios. This chapter has provided the background information necessary for creating a best practice framework, the main focus of the following chapter. 


\section{CHAPTER 3: METHODS}

\subsection{Introduction}

The purpose of this research is to determine if the CNSC is protecting the public from unreasonable risk through compliance with HIA best practices. To attain this end, a best practice framework must be devised and applied to relevant EISs of nuclear power in Ontario. This chapter first describes the methods used to identify best practices through reviewing the literature and conducting semi-structured interviews to expand on concepts derived in the literature. The conclusions from the literature review and interview process are then collated to develop the evaluation framework. Finally, the EISs selected for evaluation are justified and the review process explained.

\subsection{Research Methods}

In order to answer the central question of this thesis, whether the CNSC is ensuring the application of best practices in the EA process for nuclear power plants, four objectives must first be accomplished:

1. Establish HIA best practices for nuclear power projects from authoritative sources on HIA, HRA and PRA;

2. Create an evaluation framework based on consistently identified best practices;

3. Apply the framework to released EISs of nuclear power projects in Ontario;

4. Determine the extent to which best practices have been achieved and recognize any significant deficiencies. 
Despite the CNSC's contention of adhering to international best practices

(CNSC, 2005a), no such standard exists. This research adopts a two-tiered approach to form such a standard, through an extensive literature review and an expert interview process (See Figure 3.1).

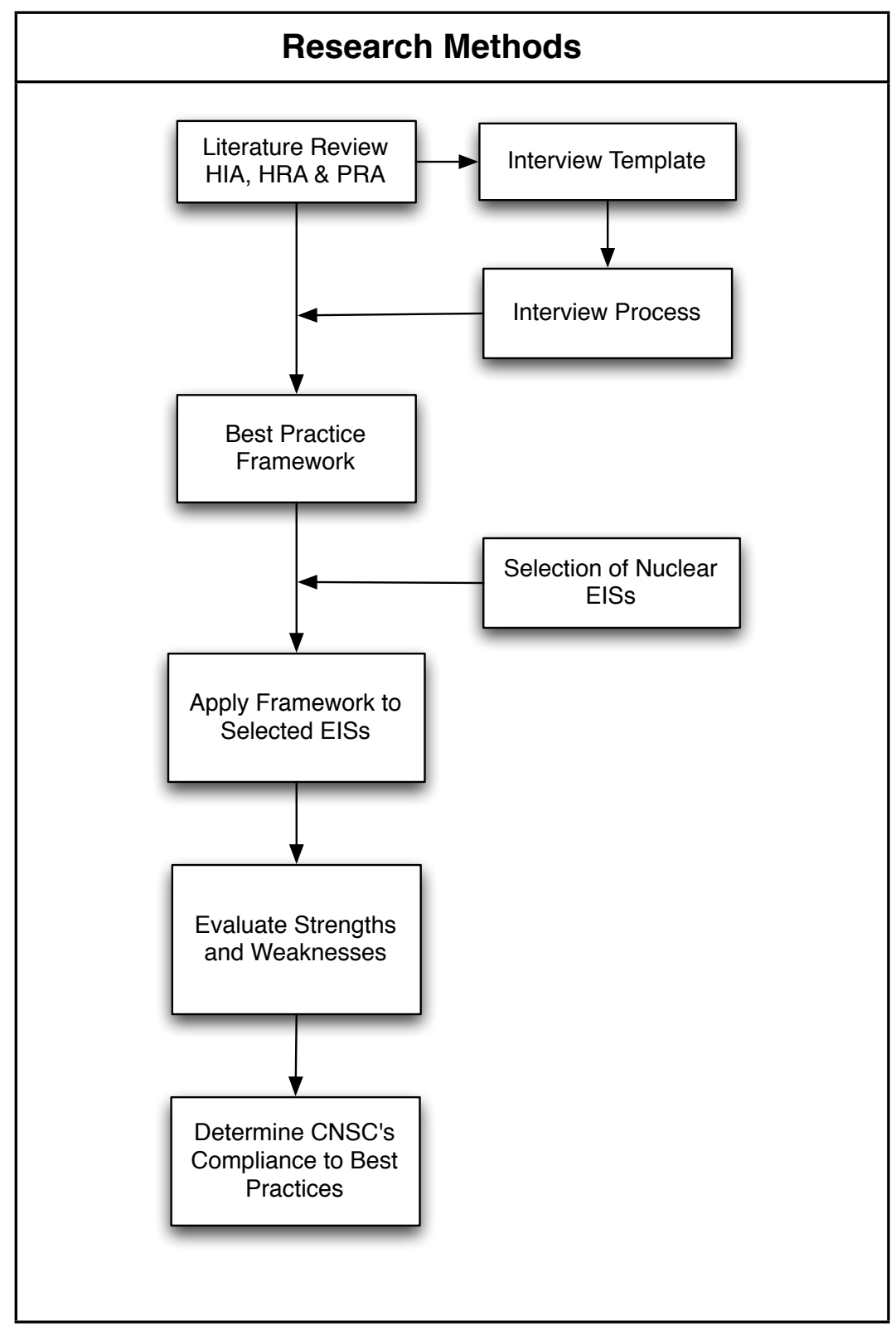

Figure 3.1 The methods used to achieve the research objectives. 
The literature review, outlined in Chapter 2, revealed three dominant research areas on which to base best practice criteria for HIAs of nuclear power projects: HIA and EA integration (for example, Davies and Sadler, 1997; BMA, 1999; Health Canada, 2004a; Bhatia and Wernham, 2008 ); HRA49 (see Harvey, 1990; Canter, 1993; Campbell-Mohn and Applegate, 1999; Jardine et al., 2003; Demidova and Cherp, 2005; Rahn and Upton, 2008; Till, 2008) and PRA (specifically, USNRC, 1990, Fullwood, 2000; Lochbaum, 2000; Garrick and Christie, 2002; Thompson, 2008b,c). The information drawn from these main sources, as well as many others, was used as the foundation for the interview template.

\subsection{Qualitative Research}

\subsubsection{Interview Template}

The collation of HIA, HRA, and PRA principles, as outlined in the preceding chapter, resulted in 18 broad interview questions/topics (See Appendix A for the interview template). All of the questions were open-ended and strived to gather a broad range of knowledge. Open-ended questions can be used as an effective means of gathering the views of individuals without presuming an answer (Seidman, 1991). Questions in the interview template addressed the public's role in HRA, the appropriate use of PRA, reactor safety issues, and the health risks of low doses to ionizing radiation, among other matters. A final question was included that urged participants to identify any additional information relevant to best practices that had not been previously mentioned. It was hoped that this last question would cover any remaining

\footnotetext{
49 Also, radiological risk assessment.
} 
issues that the interview questions failed to elicit (Sobh and Perry, 2006). The intention of the interview process was to garner the opinion of experts, thus complementing the concepts identified in the literature. The methodological assumption here is that best practices $^{50}$ are approximated by the degree of concordance among concepts in the literature and opinions of HRA/PRA authorities. Ultimately, the interviews would contribute to a deeper understanding of essential best practices for HIAs of nuclear power plants.

\subsubsection{Interview Protocol}

The interview package and desired protocol were submitted to and approved by the Ryerson University Research Ethics Board. The protocol ensured all of the necessary precautions would be taken to guarantee full confidentiality. Along with an invitation to participate, which briefly outlined the nature of the research, the interview template and consent agreement were sent to all interview candidates via email. This material allowed potential interviewees to fully comprehend both the objectives of the research and the content of the interview. Also, it would enable participants to consider topics beforehand, to ensure thoughtful responses. After the initial contact, a follow-up email was sent a week later to determine whether the individual would agree to participate and if so, schedule an interview time. In many cases, candidates quickly responded via email to agree or decline before the follow-up email could be sent. If individuals agreed to participate, a time would be scheduled for the researcher to place a telephone call. If individuals had questions about the nature of the research,

50 See Zaring (2006) for an in depth discussion of the term, "best practices". 
clarification would be given via email to ensure interviewees were comfortable with the subject matter (Meho, 2006).

Due to the global nature of nuclear and radiation research, it was unrealistic to restrict interview candidates to the researcher's locale. Instead, interviewees in a number of countries were contacted with the intent of conducting telephone interviews. Empirical research has suggested that face-to-face and telephone interviews yield results that do not differ significantly for qualitative research (Sturges and Hanrahan, 2004). Interview durations generally ranged from half to three quarters of an hour. Providing written consent was given, all interviews were recorded and transcribed by the researcher (Easton et al., 2000). Shortly after the interview, an email was sent thanking the individual for participating in the research.

\subsubsection{Participation Rates}

Potential interview candidates were identified through the literature review as published authors in peer-reviewed journals on radiation protection, HRA and PRA. While special efforts were made to contact individuals knowledgeable about CANDU reactors and the Canadian nuclear industry, an attempt was made to contact those who were not affiliated with AECL, BP, OPG or the CNSC, to avoid possible conflicts of interest. Initially, 49 individuals identified in this manner were sent the interview package via email. However, several candidates additionally recommended contacts, if they knew of an individual who would be particularly valuable to the research focus. These recommendations resulted in sending an additional 11 interview requests via email. After a total of 60 interview requests were sent, 13 telephone interviews were 
conducted, supplemented by two additional written statements; these individuals either did not feel comfortable responding verbally in English or were under time constraints. Email interviews have been suggested as a viable alternative to telephone interviews (Meho, 2006). The completion of 15 interviews falls within a reasonable range to obtain data saturation for qualitative research (Guest et al., 2006).

Since the individuals contacted were established authorities on a variety of issues, most interviewees did not feel comfortable providing a response to all questions in the interview template; therefore, more time was spent on their respective areas of expertise. The questions that were relevant to their knowledge served as a base from which to delve into more detail, a benefit of open-ended questions (Seidman, 1991). Although this approach proved successful during interview sessions, it may have initially deterred potential interviewees, thus explaining the relatively low participation rate in this study (25\%). As the actual interview template was included in the initial interview request, many candidates may have glanced at the desired topics and felt they were not suited to answer all interview questions ${ }^{51}$, consequently declining the request. Figures 3.2 and 3.3 compare the ratio of affiliations between those experts who were initially contacted to those who were actually interviewed. Although the charts appear similar, it is evident that proportionally fewer low dose radiation researchers and epidemiologists agreed to participate. While clearly experts in their fields of research, some of these individuals may not have had enough firsthand experience with the nuclear industry to warrant participation in the interview.

\footnotetext{
${ }^{51}$ Although it was stated in the invitation to participate that interviewees had the option to answer only those questions with which they were comfortable.
} 


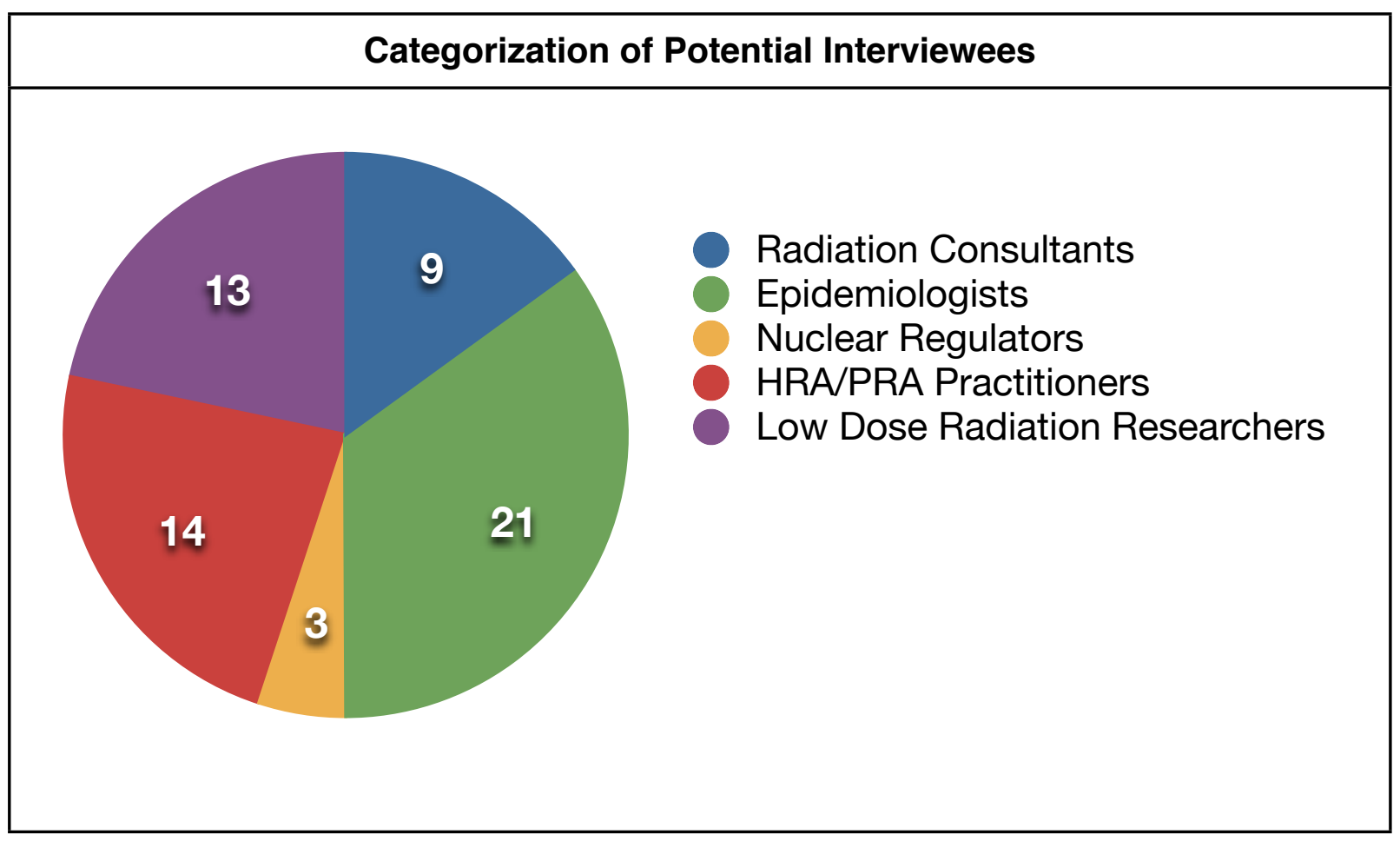

Figure 3.2. The number of potential participants from different areas of expertise.

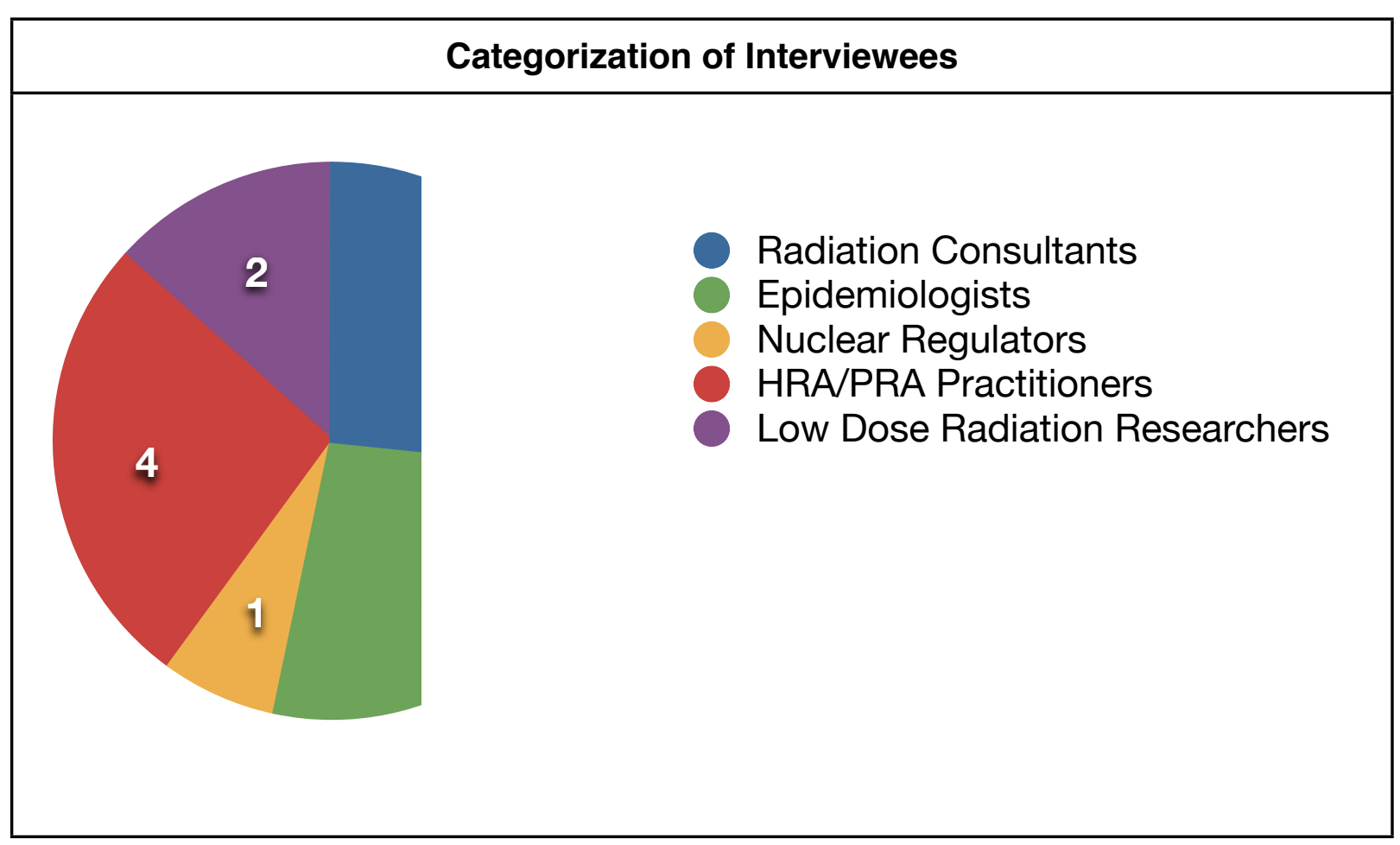

Figure 3.3. The number of interviewees from different areas of expertise. 
Despite this impediment, several indications confirm that both a reasonable number of interviews had been completed and that the pertinent individuals were contacted: (1) responses eventually exhibited a general consensus on the main concepts of the interview content, (2) the final question prompting interviewees to identify any further principles important to best practices failed to reveal any shortcomings in the interview template, and (3) participants often recommended individuals who had already been contacted (although not always successfully). Regrettably, it should be mentioned that fewer experts on PRA were successfully contacted, compared to those knowledgeable about HRA. However, the interview content was biased toward issues relating to HRA, so the desired information regarding PRA was still acquired.

With both the literature review and the interview process completed, the first research objective had been satisfied and the best practice framework could be created.

\subsection{Framework Design: A “Risk Assessment” Approach}

As outlined in the literature review, chronic and catastrophic health risks of a nuclear plant are assessed via HRA and PRA, respectively. Thus, a "risk assessment" approach is proposed for the framework. Such a design would follow the HRA paradigm and promote best practice guidance according to the four steps: hazard identification, exposure assessment, dose-response assessment and risk characterization. In addition, information concerning PRA would be embedded into the applicable HRA step. Although these two processes are separate, there are PRA objectives which coincide with those of certain HRA steps, for example, hazard 
identification $^{52}$, so integration is reasonable. Since some concepts derived from the literature on both HIA methodology and evaluation would be inappropriately integrated into the HRA steps, but were entirely necessary, an additional section pertaining to HIA "process" best practice was added. This type of framework is not a completely novel approach, as other authors have prepared criteria to help integrate HRA into the EA process (Harvey, 1990; Canter, 1993; Kwiatkowski, 1998; Demidova and Cherp, 2005).

The following subsections systematically describe the five categories of the HRA best practice framework by collating information gathered through the literature review and interview process. Since the framework incorporates 87 indicators ${ }^{53}$ of best practice, only a brief summary of the salient features of each section are given. Additionally, only the primary references are provided, to avoid cluttering the text. Those critical points which justify further discussion, either by importance or by notable inclusion/exclusion within the selected EAs, are examined in Chapter 4. Each criterion was only included in this checklist if concordance was abundantly evident among the reviewed literature and interviewed authorities. Therefore, any issues present in the framework, but not assessed during the course of an EA, would require explicit justification. Ultimately the extent of compliance with the framework would reflect the degree of conformity to best practice principles.

A summary of the criteria for each section is listed at the end of each discussion; for the sake of simplicity, references are omitted. The complete best practice framework, containing all sources, can be found in Appendix B for reference.

\footnotetext{
53 This number is on par with other EA review frameworks: Gray and Edwards-Jones (2003) formulated 81 review sub-categories to review EAs in the forest industry.
} 


\subsubsection{Hazard Identification}

The first step in HRA is to examine all the possible hazards involved in a project and to establish which have the potential to adversely impact human health (Jardine et al., 2003). The hazard identification step is critical, because it sets the terms for the entire HRA. For a nuclear power plant, a thorough description of the specific reactor technology would enable a more accurate identification of potential hazards (WHO, 1994; Demidova and Cherp, 2005). An integral part of the EA process is to assess project alternatives, and this can translate to the hazard identification step of an HRA (Steinemann, 2001). The construction of a new reactor involves selecting the desired technology from several available options, each of which presents a unique set of hazards to be assessed individually. One of the issues specific to the new generation of reactors is the concept of higher fuel burnup; in an attempt to increase profit margins, plant operators plan to irradiate the fuel for longer periods of time in order to maximize fuel efficiency (Gronlund et al., 2005). An interviewee highlighted the need to assess the specific hazards of new plants by stating "the main problem with burnup 54 is that it produces more fission products...[and]...more radioactive pollution" (Interviewee \#1 per. comm., 2009).

The life cycle of a nuclear power plant contains three main phases: construction, operation and decommissioning. As each phase presents different possible health impacts, the discussion of hazards during the EA process should not be limited to any one phase of a project (Didoc-Fikfak et al., 1999). Furthermore, the consideration of

\footnotetext{
54 It should be noted that the amount of fission products produced per unit of electricity generated is always constant; however, by achieving a higher fuel burnup, utilities will increase electricity production and thus, produce more radioactive fission products. Higher burnup also generates waste with more decay heat, having important safety implications for the storage of wastes. (Ansolabehere et al., 2003).
} 
hazards should also account for any varying emissions during each phase. To illustrate, tritium emissions steadily increase during the life-span of a CANDU reactor (Galeriu et al., 2003). This technology employs heavy water for both the moderator and coolant, which ultimately results in continually increasing neutron activation of the deuterium molecules, producing ever increasing tritium emissions (Fairlie, 2007). Additionally, the waste products differ considerably between the operation and decommissioning stages; an individual assessment of these materials is necessary. A major concern regarding the decommissioning stage is worker exposure to materials containing asbestos, on top of the routine exposure of ionizing radiation (Didoc-Fikfak et al., 1999).

An indication of the safety features of each proposed design, though not typically outlined in an HRA, should be included to ensure a meaningful assessment of the potential hazards (Demidova and Cherp, 2005). The Chernobyl incident in 1986 made the possibility of a nuclear accident a reality, prompting regulators and industry to reevaluate their safety cultures. The importance of this issue was echoed by some of the interviewees, one individual prescribing that "any new nuclear plant should have the maximum number of physical security and management measures to minimize human exposures" (Interviewee \#2 per. comm., 2009). A regulator from the UK commenting on the decision to license new plants in that country demanded "that the standards of any new plants built [there] are world-class and that they are the best that can be achieved"55 (Interviewee \#3 per. comm., 2009).

\footnotetext{
55 Indeed, the UK government has launched a multi-year project to study the same reactor designs that Canada is considering, to assess whether they represent "best available techniques" (Copplestone et al., 2009).
} 
Finally, the hazard identification stage should also include an assessment of possible accident scenarios, via PRA (The Standing Senate Committee on Energy, The Environment and Natural Resources. 2001). The decision to include PRA in the hazard identification step was predicated on the objective of a PRA: to produce source terms for different accident scenarios. Once these source terms have been identified, subsequent environmental modeling can identify exposure levels for surrounding populations. Thus, the hazard identification section is appropriate for providing an explanation of these possible release values.

PRA is a tool, albeit, a very imperfect tool, to calculate the probability of reactor core damage leading to a potentially large release of radionuclides. There are two types of events used in PRA to quantify the risk of reactor core damage: internal and external. Internal events incorporate equipment failure and human error, and external events primarily encompass natural hazards, e.g., earthquakes (Thompson, 2008b). The Nuclear Energy Agency (NEA) recently concluded that the treatment of external events in PRA varies greatly among different countries (NEA, 2009), yet studies have demonstrated core damage frequencies to be comparable to those from internal events; therefore, their risk contribution should not be undermined by a limited analysis (USNRC, 1990).

In a post-9/11 world, it is crucial to ensure that an assessment of acts of malevolence has also been included in design considerations. One area of nuclear power plants that represent a particular vulnerability to acts of terrorism are the spent ${ }^{56}$

56 "Spent" implies the fuel is no longer useful for fission (Thompson, 2003). 
fuel pools ${ }^{57}$, most frequently located adjacent to reactor containment buildings (Thompson, 2003). These ponds contain much more long-lived radioactivity than reactor cores and are surrounded by much less structural integrity than that of a containment building58. Further, the USNRC contends that the spent fuel would spontaneously ignite if the cooling water was lost, potentially releasing up to $100 \%$ of the Cs-137 in the pool (Alvarez, 2002). Therefore, external events reflecting malevolent acts should be assessed in a PRA to sufficiently demonstrate plant resilience (Thompson 2008c). Despite the seemingly esoteric nature of these PRA calculations, "the only role of a PRA in an HRA is to determine the source term, that's all” (Interviewee \#4 per. comm., 2009). PRAs need to be specific to each reactor technology, in order to produce representative source terms of potential nuclear accident scenarios: "particle characteristics such as the activity concentrations and activity or isotopic ratios are source dependent (e.g., depending on fuel burn-up)" (Salbu et al., 2004:235). Moreover, PRAs need not be employed solely for the license of new plants; one interviewee suggested that "if there had not been a proper PRA when the existing plant was put into operation, then the refurbishment point would be an opportunity to do one" (Interviewee \#5 per. comm., 2009).

Although a PRA is essential to estimate the probabilities/consequences of a nuclear accident, many of the interviewees held reservations about this technique. One individual believed that "PRA is only as good as the assumptions you put into it...[and that it is]...better than nothing" (Interviewee \#6 per. comm., 2009). Another commented:

\footnotetext{
57 Wet storage facilities are used for 'cooling' the highly radioactive used fuel for 10 years before they are sent to dry storage (OPG, 2009c).

58 Indeed, Steinhäusler (2009) recommended that spent fuel pools be housed inside containment buildings for optimal protection against terrorist threats.
} 
I think [PRAs] are useful, but my confidence is limited, because there are too many parameters involved and the confidence limits of these parameters are not really known, so you are not sure that you have the range of results which you should expect (Interviewee \#7 per. comm., 2009).

These statements emphasize the need for transparency in PRA methods, as well as in the underlying uncertainties and confidence levels in the outputs; such openness will minimize the use of unjustified assumptions ${ }^{59}$ in the PRA (Lochbaum, 2000).

\section{Hazard Identification Criteria}

-1.1 Description of proposed reactor technology?

- 1.1.1 Mentions safety features of each alternative?

- 1.1.2 Characterizes emission differences?

-1.2. Identifies all possible health hazards for each project phase?

- 1.3. Considers increasing reactor emissions (probabilistic distributions)?

- 1.4. Discussion of accident scenarios with PRA?

- 1.4.1. Includes both internal and external events?

- 1.4.2. Incorporates acts of sabotage?

-1.4.3. Defines source terms for all accident scenarios?

-1.5. Discussion of wastes?

- 1.6. Mentions types and quantities for each project phase?

- 1.7. Discusses method of long-term disposal?

Table 3.1. A summary of the best practice criteria derived for the Hazard Identification section, as outlined in the text.

\subsubsection{Exposure Assessment}

The purpose of the exposure assessment step in HRA is to model the environmental transport of the relevant contaminants and establish the doses that humans receive (Jardine et al., 2003). Paustenbach (2000:181) offered a description of the process:

59 See Lochbaum (2000) for a detailed discussion of such assumptions. 
Exposure assessment combines elements of industrial hygiene, radiological health, and air pollution and relies upon aspects of statistics, biochemical toxicology, large-animal toxicology, atmospheric sciences, analytical chemistry, food sciences, physiology, environmental modeling, and others.

An interviewee confirmed the complexity of this step by stating that "there are hundreds of parameters between the source term and the conversion to risk" (Interviewee \#4 per. comm., 2009).

Essential to the exposure assessment step is initially providing a geographical description of the proposed or existing facility site (Paustenbach, 2000; Demidova and Cherp, 2005). This site assessment should also incorporate the background levels of radioactivity. Natural and anthropogenic background radiation levels need to be established for sites undergoing new reactor construction or expansion (Muirhead, 2008). Some authorities even urge that a distinction should be made between voluntary and involuntary exposures (USEPA, 2003; Quigley et al., 2006).

Radioactivity should be monitored in all media that constitute pathways of human exposure (Till, 2008). When monitoring levels of environmental radiation, an account of the three types of radiation should be given: alpha, beta and gamma (WHO, 1994). One interviewee warned of the dangers of disregarding certain forms of radiation: "if you measure the gamma [radiation] it is low levels, but if you inhale an alpha emitter, the surrounding cells incur a really high dose (Interviewee \#8 per. comm., 2009).

One of the prominent concerns regarding emissions from CANDU reactors is that of tritium releases (Fairlie, 2007). There are many factors that enable this particular beta emitter to be extremely dangerous to human health. For instance, the ingestion of tritiated water (HTO) will inevitably be converted into organically bound tritium (OBT), 
which entails a longer residence time in the body, and thus, more opportunity to cause harm (Fairlie, 2008). Therefore, this radioisotope deserves special attention in the exposure assessment section in an Ontario HRA.

Previous environmental monitoring data from existing facilities may be used to verify environmental modeling of future releases (Paustenbach, 2000). This is important to assist environmental modeling of all the contaminants, outlined in the hazard identification step, to ultimately determine human exposure levels (Huang and Batterman, 2000; Zou et al., 2009). An interviewee discussed the various pathways of human exposure to ionizing radiation:

One is by breathing it, one is through your skin, one is through drinking water and another one is eating food. Of those four, the food one is by far the most important- it's responsible for about three quarters of the dose (Interviewee \#6 per. comm., 2009).

Although comprehensive modeling of radionuclide transport through the environment is important, accurate assessments of human exposure rely on people's behaviours, not just levels of contamination in the environment (ICRP, 2006). Acquiring this information involves consulting the public and verifying whether any group of individuals engage in activities that make them particularly vulnerable. One interviewee asserted that it is important to assess:

The habits of people, where they get their drinking water from and where they get their foodstuffs from. Depending on the potential for exposure, it may be necessary to do some actual survey work to ask people how they behave (Interviewee \#3 per. comm., 2009). 
It is of little debate whether concerns over nuclear reactor emissions pertain to radioactive pollution; however, non-radioactive chemicals are also released in the gaseous emissions from coolant stacks. Such harmful chemicals include hydrazine, which reacts with dissolved oxygen to prevent boiler corrosion (Winfield et al., 2006). The dangers of these discharges should not be overlooked or undermined by strictly focusing on radioactive releases during an HRA. This was validated by an interviewee:

I actually think it's important to look at not just radioactivity, I think nowadays we should be looking at any chemical emissions that are happening or occur with the operation of the facility (Interviewee \#9 per. comm., 2009).

In addition to identifying any unique groups of individuals who are subject to relatively higher exposures, the HIA literature stresses the need to characterize the health of the exposed population as a whole (Parry and Kemm, 2005; Cole and Fielding, 2007). As in the EA process, in which the baseline of the environment is measured to assess the incremental stresses added by a proposed project, the health of a population should be examined for the same purpose (Health Canada, 2004a). With respect to populations exposed to nuclear power plant emissions, an interviewee described the most crucial information to obtain:

What information do you have on a disease among that populationwhat's the cancer rate, what's the background incidence of cancerincidence and mortality; what is the kind of cancer, specifically, that the population incurs...if I were a facility about to start up, this is the kind of information I absolutely gather in critical detail (Interviewee \#4 per. comm., 2009). 
Until this point, the discussion on exposure assessment has addressed the surrounding population, as opposed to the workers' exposure. The quality of this analysis is paramount; Nieuwenhuijsen et al. (2006) noted occupational settings constitute the most threatening exposure scenarios. Higher occupational exposures can also adversely affect the public, as workers may expose their families to harmful chemicals brought home on their clothing (Buckely et al., 1989). Furthermore, Paustenbach (2000) observed that sometimes the average exposure may not be as critical, when compared to peak exposure values. Higher exposure settings for nuclear workers are expected during refurbishment and decommissioning activities, due to the removal of various safety barriers (Dodic-Fikfak et al., 1999). Instead of using average worker exposure values, a probabilistic approach may be more appropriate. This approach uses a distribution of different exposure values, the frequency of each value corresponding to its probability; i.e., more common exposure values would appear more often in the range. A computer program then repeatedly picks values at random until a range of exposures and probabilities are revealed (von Stackelberg and Burmaster, 1994.). This distribution describes more realistic exposure levels to which workers will be subject, as opposed to averaging out the high and low values. This technique is known as a Monte Carlo analysis and is regarded as a vast improvement on traditional point estimates (Finley et al., 1994).

The criteria outlined in this stage will help ensure the attainment of best exposure estimates, resulting from all hazardous reactor emissions. 


\section{Exposure Assessment Criteria}

- 2.1. Exposed population identified?

-2.1.1. Geographic description of site?

- 2.1.2. All possible exposure pathways included?

- 2.1.3. Consulted public about habits?

- 2.1.4. Vulnerable groups identified?

- 2.1.5. Environmental fate of radionuclides considered?

- 2.2. Describes baseline health status of exposed population?

-2.3. Evidence of environmental monitoring for baseline contamination?

- 2.3.1. Use of these data in HRA calculations?

- 2.3.2. Use of these data to verify environmental modeling?

- 2.3.3. Distribution of radiation exposures used for public/worker exposures?

- 2.3.4. Upper and lower dose ranges given for workers?

- 2.3.5. Dosimetry for workers practiced?

- 2.4. All sources of radiation considered?

- 2.4.1. Voluntary vs. involuntary exposure distinguished?

- 2.4.2. Explicit section on tritium?

- 2.4.2.1. Differentiates between HTO and OBT?

- 2.5. Non-radioactive hazardous emissions assessed?

- 2.5.1. Baseline concentrations established?

- 2.6. Addresses half-lives of radionuclides?

- 2.6.1. Geographic and time boundaries justified?

- 2.6.2. Addresses potential harm to future generations?

Table 3.2. A summary of the best practice criteria derived for the Exposure Assessment section, as outlined in the text.

\subsubsection{Dose-response Assessment}

After the exposure assessment has been completed, it is necessary to categorize the dose-response relationship of the various toxins. This step essentially answers the question: What is the probability of a toxic response at the doses predicted by the exposure assessment (Rahn and Upton, 2007)? This stage of an HRA involves reviewing the appropriate dose-response mechanisms, and then applying these models to the exposed population.

With respect to ionizing radiation, several national and international groups periodically analyze the latest research findings and update the assumed radiation risks 
at various doses. By far, the most recognized authority on this matter is the ICRP. Nevertheless, many experts on low level radiation question the validity of their risk estimates, due to the inconsistency of some epidemiological findings with the accepted risk models (Busby et al., 2009). Other notable groups, which produce risk estimates of their own, include the United Nations Scientific Committee on the Effects of Atomic Radiation (UNSCEAR, 2006), the United States Environmental Protection Agency (USEPA, 2008), the National Academy of Science's (NAS) report on the Biological Effects of lonizing Radiation (NAS, 2006), and the Health Protection Agency's Advisory Group on lonising Radiation (HPA, 2007), among others. Despite the seeming abundance of international groups providing risk estimates, interviewees still exhibited skepticism, one individual even asserting that "there is no reliable baseline of radiation protection" (Interviewee \#10 per. comm., 2009). Nonetheless, the most recent (and precautionary) guidance from one of these authorities should be used to base the doseresponse relationship (The Standing Senate Committee on Energy, The Environment and Natural Resources, 2001). Alternatively, relating to the Precautionary Principle, the most stringent risk estimates could be adopted from collectively reviewing the data provided by these different groups.

When modeling the dose-response relationship, the selected model of low level radiation should be explicitly mentioned. The previously listed authorities all suggest a precautionary linear-no-threshold (LNT) response as the most appropriate approach (NAS, 2006). Although other response curves exist, international scientific bodies maintain that there is currently insufficient evidence to render the LNT theory invalid (NAS, 2006). Some interviewees commented on this matter: 
There has never been to my knowledge, any credible research done by...epidemiology that shows a hormetic effect; it's the opposite. We have only evidence that any increase above the unavoidable radiation that all of us get from the earth and from the cosmic radiation...has detrimental effects, so the threshold idea has very little credibility (Interviewee \#11 per. comm., 2009).

Another remarked:

I think in an HRA, it makes sense to default on a LNT approach that says we are not going to assume that there is any dose to which there are no effects...Any small increase in dose will be associated with a small increase in cancer risk and that seems to me to be the most prudent approach (Interviewee \#9 per. comm., 2009).

One crucial point that resonated throughout the HIA literature was that, even at the most basic level of an $\mathrm{HIA}$, a literature review must be performed on the possible hazards from the proposed project (Davies and Sadler, 1997; BMA, 1999; Health Canada, 2004b; Demidova and Cherp, 2005; Bhatia, 2007). With respect to nuclear power projects, this review should include information on the health risks of ionizing radiation (the previously mentioned organizations are obvious sources for this information) and any studies pertaining to the specific reactor technology or exposed populations. Supplementing this discussion should be an identification of the uncertainties in the state of knowledge, as well as the limitations of any specifically mentioned studies (Richardson et al., 1997; Briggs et al., 2009; Nussbaum, 2009).

The LNT curve for ionizing radiation is mostly predicated on the Life Span Study of the atomic bomb survivors. This theory posits that increasing doses directly correspond with increasing cancer risks (NAS, 2006). Accordingly, the results of an 
HRA determine the increased incidence of cancer in the exposed population, determined by the dose levels. However, research demonstrating other health detriments caused by ionizing radiation is mounting (Richardson, 2009; Shimizu et al., 2010). An interviewee added further insight:

It is not just cancer and birth defects, certainly brain damage, cardiac damage. A lot of the inhalation of isotopes has produced damage to the lining of the arteries and veins and caused serious cardiac and brain abnormalities as a result of damage to the circulatory system...There has hardly been any system that has not been adversely affected (Interviewee \#12 per. comm., 2009).

While the HRA paradigm has not yet been updated to consider non-cancer effects caused by ionizing radiation, omitting these other impacts altogether would mislead the public about the potential range of project health impacts (Cole et al., 2005).

For determining the doses from chronic exposure to ionizing radiation, all HRA calculations should be present in the EA (Demidova and Cherp, 2005). As the issue of transparency was underscored in the HIA literature, all values and assumptions used for the dose calculations should be explicitly given (Health Canada, 2004b, Briggs et al., 2009). The exposure assessment step analyzes the environmental transport of the emitted radionuclides, in order to measure human exposure. Once these exposures are quantified, dose conversion factors (DCFs) are then used to account for different types of radiation and sensitivities of different organs. DCFs are constantly evolving and should be indicated alongside HRA calculations to ensure the application of the most updated values (Fairlie, 2005). Additionally, the ICRP recommends that external and internal doses be calculated separately (ICRP, 2006). 
One of the most prominent criticisms of HRA is the use of the Reference Man for radiological protection purposes. That is, the purpose of an HRA is to demonstrate that a hypothetical individual's exposures to a certain contaminant, or subset of contaminants, will fall within regulatory guidelines. This individual is also known as the Maximally Exposed Individual (MEI) (Adler, 2004). All of the various ingestion, inhalation and dose-response data are based on the Reference Man, who is "a hypothetical 20 to 30 year old Caucasian male" (Makhijani, 2008:7). This practice is untenable for the purposes of assessing risks to ionizing radiation, which should be calculated for males and females separately. For a lifetime exposure to the annual dose limit, the BEIR-VII outlines the risk of cancer incidence as nearly double for women (NAS, 2006:312). Assessing the risks solely for males would understate the effects for women. This fact carries particular weight when considering occupational exposures, which tend to be higher than public doses.

Fortunately there is an emerging departure from this practice, as HRAs are increasingly used to determine risks for more than just young Caucasian males. Perhaps more important than calculating risks independently for men and women is to determine risks for children. This is especially true for ionizing radiation. Studies have shown children to be most sensitive to the dangers of ionizing radiation for a number of different reasons (Busby and Fucic, 2006; Kaatsch et al., 2008). The ICRP has recognized this issue and made the necessary recommendation to assess risks separately for the following age groups: 0-5 years, 6-15 years and 16-70 years (ICRP, 2006). One interviewee voiced her concern on this matter:

It is ridiculous to use the standard man when there is such a 
diversity in sensitivity. In fact it is now being proposed by doctors in Germany and elsewhere that we use the standard embryo or the standard fetus, because they are the ones who are going to be most affected (Interviewee \#8 per. comm., 2009)

The previous discussion summarized the necessary components to be incorporated in the dose-response assessment of chronic exposures. To examine the effects of acute exposures, a Level 3 PRA ought to be performed. By using the source terms generated in the Level 2 phase of a PRA, radionuclide transport may be modeled using meteorological conditions to predict doses to the surrounding population. The resulting adverse health impacts, expressed as magnitudes and probabilities, should be represented by such factors as early fatalities, latent cancers and even short or long term abandonment of land (Thompson, 2008b). Garrick and Christie (2002) have postulated that once the source term has been determined, estimating offsite consequences is much less demanding. This practice is hardly revolutionary, as an interviewee recounted:

The council on environmental quality, which is an arm of the White House, made a ruling that for EISs of new plants, there needed to be a Level 3 [PRA] performed and the results published, and so a number of those were published in the 80s. (Interviewee \#5 per. comm., 2009).

The range of offsite consequences, according to the different magnitudes of identified source terms, should be revealed to fully appreciate the potential acute health risks of a project. This practice of assessing accident scenarios is aligned with both the HIA literature (Mueller, 1996; BMA, 1999; Health Canada, 2004a; Demidova and Cherp, 2005) and CEAA (CEAA, s. 16(1a)). Furthermore, "HIA places a high value on 
addressing all potentially significant outcomes, even if they are difficult to ascertain" (Cole et al., 2005:383).

\section{Dose-response Assessment Criteria}

- 3.1. Most recent dose-response data used?

- 3.1.1. Describes low dose-response radiation model?

- 3.1.2. States all important health effects from ionizing radiation?

- 3.1.3. Doses for males and females?

- 3.1.4. Dose Conversion Factors included?

- 3.1.5. Different age groups used to calculate doses?

- 3.1.6. Determines most significant radionuclide(s) for human health?

- 3.2. Mentions any studies of exposed population/reactor technology (lit. review)?

- 3.2.1. Discusses limitations of findings?

- 3.3. Differentiates between internal and external doses?

- 3.3.1. Alpha, beta, gamma radiation explained?

- 3.3.2. Describes bioaccumulation in certain organs?

- 3.3.3. Discusses possible synergistic relationships of ionizing radiation?

- 3.4. Predicts potential offsite health consequences of accidents (Full Level 3 PRA)?

- 3.5. Identifies most significant non-radioactive emission?

Table 3.3. A summary of the best practice criteria derived for the Dose-response Assessment section, as outlined in the text.

\subsubsection{Risk Characterization}

Having completed the first three stages of the HRA, enough information should be available to properly convey the risks of the project. The main objectives of the risk characterization step in a traditional HRA are to first, verify whether risks posed from the project are within acceptable levels- or in terms of radiation protection parlance, permissible doses- and second, to discuss the uncertainties and degree of precaution appropriate to the risks (Jardine et al., 2003). This risk characterization step should present an unbiased presentation of the calculated risks and not try to underestimate the potential impacts of the project (Birley, 2003). 
Before any explanation of the characterization of risk, the acceptable risk levels must be explicitly stated (Campbell-Mohn and Applegate, 1999). There are two different levels of risk that pertain to nuclear power HRAs: the annual limit of $1 \mathrm{mSv}$ of anthropogenic emissions of radiation (CNSC, 2008d) and the specific reference doses (annual limits) for each non-radioactive chemical (Tickner, 2007). As previously mentioned, the emphasis on the MEI is fading. Along with the different age groups to be assessed, this same ICRP task group advised HRAs to consider exposures levels to 'a few tens of people' (ICRP, 2006). The USEPA has also put forth guidance to identify any groups of people who incur higher risks through certain activities or behaviours (USEPA, 2008). More focus is starting to be placed on the protection of populations, especially real exposed populations, than solely on the hypothetical MEI (Adler, 2004). For PRAs, the calculated risk levels should demonstrate that offsite health consequences are below an accepted threshold, and that the probability of a large-scale radioactive release is below one in a million per operating year of each unit (CNSC, 2008c).

The quantification of uncertainties was added to the four steps of a traditional HRA by the NAS in the 1980s (Ozonoff, 1994). Both the HRA and PRA literature strongly suggest that uncertainties and confidence levels be clearly presented with all calculations (USNRC, 1990; Paustenbach, 2000; Thompson, 2000; Garrick and Christie, 2002; Briggs et al., 2009). Harvey (1990) argued that an explicit description of uncertainties and underlying biases would only serve to further bolster the public's trust in an HRA. In addition, sensitivity analyses are necessary to demonstrate the robustness of models under different assumptions and to check for "cliff edge" effects 
(Briggs et al., 2009). An interviewee explained the necessary steps of achieving a transparent HRA process:

We would explain how we arrived at those decisions, what the results of our HRAs are, how we've done the modeling and the assumptions we've made, the doses that lead to the most exposed people and how that compares with international and national limits (Interviewee \#3 per. comm., 2009).

Along with the presentation of risks, it is appropriate to identify the strategies for which risks will be mitigated, as well as the necessary monitoring activities to ensure impact predictions are validated (Parry and Kemm, 2005; Quigley et al., 2006). This is also a good opportunity to demonstrate how the Precautionary Principle, emphasized in CEAA (CEAA, s. $4(1 \mathrm{a}))$, has been incorporated in the HRA uncertainties and proposed mitigation activities (Jardine et al., 2003). The various environmental models of radionuclide transport that were created during the EA and licensing stage of a new plant/refurbishment should be followed up with real environmental data (Petticrew et al., 2007). This can include bio-monitoring data of radiation levels in organisms which may be important pathways to human exposure. Bio-monitoring can present a more accurate picture of the total radiation exposure from all sources (USEPA, 2003; Nieuwenhuijsen et al., 2006). An interview candidate provided insight on the value of bio-monitoring:

There have been a few instances of biaccumulation of organic tritium...that were unexpected in fish...This has altered some of the models we use for organic tritium (Interviewee \#3 per. comm., 2009). 
One interviewee took the concept of environmental monitoring even further by promoting the use of the monitoring program at the Los Alamos National Laboratory in New Mexico for commercial nuclear power plants, explaining: "it's live time, updated once a week and that's the way the public ought to be informed about what's happening at a facility" (Interviewee \#4 per comm., 2009). These practices of monitoring the environment for radiation present a better picture of the doses surrounding populations will be receiving without actually conducting bio-monitoring of humans. Accompanying these data, and strongly emphasized in the HIA literature, should be an outline of various health indicators to be monitored in the exposed population (Davies and Sadler, 1997; BMA, 1999; Health Canada, 2004a; Bhatia and Wernham, 2008). As outlined above in the dose-response assessment discussion, these indicators should involve various cancer incidence and mortality information, as one interviewee maintained: "exposure to fission products is a risk to all types of cancer" (Interviewee \#2 per. comm., 2009).

Throughout the history of radiation protection, an accepted term has emerged that represents the current safety culture of radiation-containing environments: As Low As Reasonably Achievable (ALARA). Contrasting with always using the highest safety precautions, the concept of ALARA maintains that "licensees are expected to reduce doses where this can be done without significant expenditures" (CNSC, 2004:3). The CNSC also suggests that "in some situations, a decision is required on whether it is economically justifiable to take action to reduce dose levels" (CNSC, 2004:3) These statements provide ambiguity as to the extent of mitigation measures required, so long as exposures are below permissible levels for occupational settings. Therefore, the risk 
characterization section presents an ideal opportunity to outline the planned techniques to reduce radiation exposure to both workers and public during all stages of the project (Demidova and Cherp, 2005).

Finally, the HIA literature places the attainment of sustainable development in high regard (Health Canada, 2004a; Quigley et al., 2006). The strong link between sustainable development and human health has long been established (Davies and Sadler, 1997). As such, the acceptability of a nuclear power project should also be presented in terms of sustainability. An appropriate sustainability assessment of nuclear power projects would include the depletion of uranium resources and the production of extremely long-lasting nuclear waste, among other issues (WHO, 1994; Krewitt, 2002).

\section{Risk Characterization Criteria}

- 4.1. Acceptable risk defined?

- 4.1.1. Identification of any individuals approaching acceptable risk limits?

- 4.1.2. Determines if 'a few tens of people' likely approaching limits?

- 4.1.3. Risks below HRA standards?

- 4.1.4. Risks below PRA standards?

- 4.2. Addresses perceived risk (radiophobia)?

- 4.2.1. Explains possible psychological effects?

- 4.3. Describes various uncertainties, assumptions and confidence levels?

- 4.3.1. In all calculations?

- 4.3.2. In all models?

- 4.4. Formal environmental monitoring discussed?

- 4.4.1. Monitoring of health indicators proposed?

- 4.4.2. Any bio-monitoring of radiation levels in non-human biota?

- 4.4.3. Real-time monitoring available to public?

-4.5. Commitment to specific formal mitigation measures?

- 4.5.1. Describes reduced health risks due to mitigation?

- 4.6. No biases in presentation of material?

- 4.6.1. Precautionary principle addressed?

- 4.7. Addresses sustainability of project?

Table 3.4. A summary of the best practice criteria derived for the Dose-response Assessment section, as outlined in the text. 


\subsubsection{Process}

An additional section in the best practice framework was provided beyond the four stages of a traditional HRA, pertaining to the methodology of the entire HIA process. General concepts included here were strongly supported in the HIA/HRA/ PRA literature, but were inappropriate to include other sections.

To begin, some relatively simple, but absolutely essential, indicators of a sound HIA process are addressed. The HIA process should be completed before any action is taken; in other words, knowledge gained during the HIA process should be available to alter the project, if necessary (Birley, 2003; Parry and Kemm, 2005). At the outset, the HIA should accept the broad WHO definition of health, the endorsement of which will ensure a more inclusive HIA (BMA, 1999). Another simple indicator is the presence of non-technical summaries, which briefly outline the potential health risks from the project (Lee et al., 1999). This is very useful for nuclear power EAs, in particular, which usually contain hundreds, if not thousands, of pages in the main document, not to mention numerous appendices and technical support documents.

Critical to any HIA/HRA/PRA process is the practice of independent peer review (Applegate, 1995; Birley, 2003; Jardine et al., 2003; ICRP, 2006; Thompson, 2008c; Till, 2008). Such reviews will encourage the highest standards, through the identification of any weaknesses, omissions or biases. The term "independent" means that a review must be performed by an individual or group who is not affiliated with the project and is without conflicts of interest. Interviewees held strong beliefs on independent review, asserting that only certain individuals are truly capable of performing this kind of review:

It's absolutely paramount that you have independent review 
of health risk assessments...because the people who do the health risk assessments are all people in the pocket of the nuclear industry (Interviewee \#1 per. comm., 2009).

Another interviewee elaborated:

Theoretically, it could be anyone who's an expert. In reality, because of the politicized nature of radiation, i.e., in terms of nuclear weapons and nuclear plants...anyone affiliated with government and the nuclear industry should not be a peer reviewer (Interviewee \#2 per. comm., 2009)

Likewise, if the EA is selected to be a panel review, the panel should consist of three individuals who do not possess any obvious industry ties or conflicts of interest (Birley, 2003; Huff, 2007).

An integral part of any HIA process is public participation (Kearney, 2004). This is an area of chief importance for any HIA, but particularly for nuclear power projects; one interviewee revealed: "this is where the nuclear industry has blown it (Interviweee \#4 per. comm., 2009). Without being too prescriptive, there are some basic steps that help foster effective public consultation. Perhaps most important is attempting to get the public engaged as early as possible in the HIA process and trying to gain their trust (O'Connor and van den Hove, 2001). Areas of concern for nuclear power plants that may arise early, such as the sufficiency of emergency evacuation plans (Rogers, 1998), can be mitigated by holding workshops on specific topics and disseminating the results (BMA, 1999; Parry and Kemm, 2005). Additionally, the EIS should feature a section which acknowledges the most prominent public concerns and lists how these were addressed in the HIA process (Health Canada, 2004b; Demidova and Cherp, 2005). 
A final note highlighted by a number of interviewees, typically the only issue arising from the final interview question, was the concept of free access to health information of the exposed communities. This can provide validation of HIA predictions (Petticrew et al., 2007; Veerman et al., 2007) and enable independent scientists to conduct objective epidemiological studies (Scherer et al., 1994; Hansell and Aylin, 2003). The following excerpts convey these sentiments: "the most important thing is independent access to health data, particularly cancer and leukemia data in small areas "(Interviewee \#1 per. comm., 2009); "there needs to be lots of sunshine on the data, it should be available for anyone who wants to use it and in a timely manner" (Interviewee \#12 per. comm., 2009); "cancer registries should open for independent research" (Interviewee \#7 per. comm., 2009).

\section{Process Criteria}

- 5.1. Broad WHO definition of health given?

- 5.2. HRA started before any action?

-5.3. Communicates risks clearly?

- 5.3.1. Non-technical summaries?

- 5.3.2. Various dose metrics and units defined?

- 5.4. Evidence of HRA peer review process?

- 5.4.1. Reviewed by independent groups?

- 5.5. Evidence of PRA peer review process?

- 5.5.1. Reviewed by independent groups?

- 5.6. Independent health experts consulted?

- 5.7. Early evidence of stakeholder involvement?

- 5.7.1. Public concerns identified in the HRA?

- 5.7.2. Activities/results of any workshops disseminated?

- 5.8. Separate section for HRA?

-5.9. Funding of any new studies to address ongoing public concerns?

- 5.10. Confirms refurbishment would satisfy international safety standards?

- 5.11. Emergency Evacuation Plan guided by Level 3 PRA?

- 5.12. If EA is a panel review, independent reviewers selected?

Table 3.5. A summary of the best practice criteria derived for the Dose-response Assessment section, as outlined in the text. 
This concludes the discussion of the best practice framework, thus satisfying the second research objective. The next section discusses the selection of EAs for review and the evaluation protocol.

\subsection{Application of Framework}

\subsubsection{Selection of EAs}

The best practice framework discussed in the previous section pertains to HIAs for restarting, refurbishing and/or constructing nuclear reactors; therefore, only EISs of these projects would be appropriate for review. The list of cancelled/completed/ongoing EAs on the CNSC website was consulted to select the appropriate EAs (CNSC, 2009f).

Shortly after CEAA was brought into force, the IIPA was carried out in 1997 and prompted OPG to put the Pickering A and Bruce A reactors into a temporarily laid up state (Arai, 2001). The subsequent return to service of these reactors produced screening level EAs in 2000 and 2002, respectively. Additionally, the aging reactors at Bruce $A$ and Pickering $B$ prompted BP and OPG to initiate EAs for refurbishment, which were released in 2005 and 2007, respectively. These four EISs were selected for review with the framework.

Additionally, the IPSP, used as a decision making tool for Ontario's energy future over the next 20 years, called for the expansion of nuclear power to meet rising demand (OPA, 2008). Consequently, the proposals for new reactors at the Bruce and Darlington generating stations have resulted in EAs at the panel review level for each project. The EISs were released in 2008 and 2009 and were also selected for review with the framework. 
It is worth mentioning that the Gentilly Unit 2 reactor completed a refurbishment EA in 2006. Unfortunately, the proponent, Hydro-Quebec, released the EIS solely in French, rendering a review of this document unfeasible for the researcher. An appraisal of that EIS would be appropriate for further research with the evaluation framework in jurisdictions other than Ontario. Additionally, the CNSC renewed an operating license to New Brunswick's Point Lepreau nuclear plant in July 2006. Even though New Brunswick Power would be conducting refurbishment activities during that period, which has previously required an EA, these were scheduled to occur during a planned maintenance outage. This detail was enough to convince the CNSC that there would be no trigger for CEAA (CNSC, 2007b). Consequently, the list of six EISs that were selected for review constitute all restart/refurbishment/new build nuclear power EAs (in English) that have been initiated since the promulgation of CEAA in 1995.

\subsubsection{Evaluation Method}

To satisfy the third research objective, the EISs were reviewed in chronological order, beginning with the Pickering A Return to Service and finishing with the Darlington New Build. Fortunately, several of the EISs and corresponding appendices were available online at the BP and OPG websites. However, the Pickering A Return to Service EIS was not available electronically and had to be reviewed at the Toronto Urban Affairs Public Library. The Radiation and Radioactivity Technical Support Document (TSD) was obtained electronically from SENES Consultants Limited for further verification on the Pickering A main EA report. Additionally, the Bruce A Restart EIS was acquired from the CEAA registry in electronic format. It should be noted that 
an unsuccessful attempt was made to obtain the Radiation and Radioactivity TSD for the Bruce A Restart. Despite this obstacle, the main document proved to provide the necessary information for evaluation.

The Lee et al. (1999) review package and protocol have provided a benchmark for the EA review process (Gray and Edwards-Jones, 2003; Demidova and Cherp, 2005). Their review protocol, and some of their evaluation criteria, were used as a starting point for the evaluation process in this research. Prior to the review of the first EIS, the researcher made sure to be adequately familiarized with the best practice evaluation framework. Adhering to the guidance of Demidova and Cherp (2005), the following information on the EA was initially recorded: year, title, type (e.g., screening), location and names of the proponent, RA and organization that prepared the EA. All sections of the main EA document were then examined; notes were taken on areas pertinent to the criteria in the framework. The points in the framework were most likely not addressed sequentially (or in some cases, at all), which made taking notes more practical. After the main EA document had been scanned, any relevant appendices or TSDs were then examined; for the purposes of HRA and PRA review, this usually entailed examining the Radiation and Radioactivity; the Malfunctions, Accidents and Malevolent Acts; and the Human Health TSDs. Although consulting these documents usually provided a wealth of information from which to draw, two points in particular, required further research efforts. Criterion 5.5 inquired as to whether the PRA had been independently reviewed. An independent review of PRA would be essential prior to EA approval, so it was necessary to determine if it had already been subjected to third party 
review, or at least by the $\mathrm{CNSC}^{60}$. Likewise, the last criterion, 5.13 , questions the independence of the panel members in a review panel EA; this information was available on the CNSC website. For the remainder of the criteria, the approach recommended by Lee et al. (1999) was adopted: if information on a specific criterion was missing from the EIS, it was assumed not to have been satisfied. After notes had been taken on the EIS and supporting materials, the checklist was then completed.

Unlike the Lee et al. (1999) review package and others (Gray and EdwardsJones, 2003; Demidova and Cherp, 2005), the best practice framework derived in this research adopted a binary evaluation approach. The reason for this methodological variation is that the framework can serve two purposes: it can be used prior to or during a project, as HIA guidance material, or after a project, as evaluation criteria. The design of this framework is specific to nuclear power projects, allowing the criteria to be much more focused than general EA review material (Lee et al., 1999). This checklist prompts the reviewer to enter "Y", if a criterion has been included, and " $\mathrm{N}$ ", if a criterion has been omitted. Similarly, the framework allows proponents, or interested parties, to go through the list during an HIA to determine if each point has been considered and if not, to ensure any omission is explicitly justified within the HIA. Thus, a more objective approach is possible to assessing the presence or absence of best practices than administering letter grades ${ }^{61}$.

After all the essential information on a particular EA was collated and recorded, the checklist was then filled out as previously described. If a certain criterion was not

\footnotetext{
60 Ideally, the PRA peer review process should be conducted before the release of an EIS so that it may be referenced in the document.

${ }^{61}$ Admittedly, letter grades were generated for each section in this research, but these reflected the proportion of satisfied criteria and made it easier to observe general trends.
} 
applicable to the EA in question, it was omitted from the analysis and "N/A" was recorded. At the conclusion of each of the five sections, the "Y" responses were totaled to provide a rough indication of the quality of that section. However, it is important to mention that each best practice criterion is not of equal importance; although the total of each section would generally be indicative of HRA performance, the presence and absence of specific indicators do need to be taken into account. The following chapter will comment on the trends for the criteria of higher importance.

\subsection{Summary}

This chapter presented an overview of the methods that yielded the best practice evaluation framework. The interview process was discussed; the subject material of the interview template and the selection of the interview candidates were described. A combination of the best practice concepts identified in the literature review and the information acquired during the interview process were then used to devise the framework. Direct quotes were identified from interviewees to underscore salient issues. A general breakdown of each of the five sections was provided to familiarize the reader with the evaluation criteria. The specific EAs chosen for review were then outlined and the evaluation protocol explained. 


\section{CHAPTER 4: RESULTS AND DISCUSSION}

\subsection{Introduction}

This chapter provides an in-depth discussion of the results of each section of the evaluation framework: hazard identification, exposure assessment, dose-response assessment, risk characterization and process. Strengths, criteria that were accomplished in every or nearly every EA, are addressed first, followed by weaknesses, criteria that were absent in every or nearly every EA. This chapter concludes with a discussion on the overall performance of HRA and PRA, with respect to the best practice criteria. For the purposes of disambiguation, each reviewed document is referred to as an Environmental Impact Statement (EIS), each of which contains aspects of Health Impact Assessment (HIA), Health Risk Assessment (HRA) and Probabilistic Risk Assessment (PRA).

\subsection{Hazard Identification}

As noted in Chapter 3, the purpose of the hazard identification step is to list all possible hazards associated with a project and then determine which are relevant to surrounding human populations. The results of this section from reviewing the six nuclear power EISs are shown in Table 4.1. Note that the totals may be slightly different as not all criteria were completely relevant to each project (see Table 3.1 for a summary of the best practice criteria). 


\begin{tabular}{|l|c|}
\hline \multicolumn{1}{|c|}{ EA } & $\begin{array}{c}\text { Hazard Identification } \\
\text { Results }\end{array}$ \\
\hline Pickering A Restart & C \\
\hline Bruce A Restart & $7 / 11$ \\
\hline Bruce A Refurbishment & $7 / 11$ \\
\hline Pickering B Refurbishment & B \\
& $8 / 11$ \\
\hline New Bruce & B \\
& $8 / 11$ \\
\hline New Darlington & B \\
& $9 / 12$ \\
\hline
\end{tabular}

Table 4.1. The results of the Hazard Identification section.

\subsubsection{Hazard Identification Strengths}

Aside from the criteria pertaining to PRA, the hazard identification stage was one of the relatively better performed steps in the reviewed EISs. The restart and refurbishment EISs included lengthy descriptions about the reactor technology and safety features specific to each plant. This practice was replicated for each of the proposed new reactors at the Bruce and Darlington sites. The Bruce EIS presented the actual core inventories for each reactor alternative, thus permitting an appreciation of the different core compositions (BP, 2008e). Regrettably, the Darlington EIS omitted this information, preventing such a comparison. Nevertheless, both EAs employed a Bounding Scenario approach- referred to as a "Bounding Plant Envelope" in the new Bruce EIS (BP, 2008c) and as a "Plant Parameter Envelope" in the Darlington EIS 
(OPG, 2009d)- for the purposes of performing an HRA. This technique collates the highest quantity of gaseous and liquid release parameters associated with the normal operation of any one reactor technology. These values were then used to produce a hypothetical reactor core inventory from which to model routine environmental releases. The objective of this procedure is to demonstrate that chronic exposures will be kept acceptably low, regardless of which technology is finally chosen.

Identifying health risks separately for each phase of the project, an important criterion as specified in the HIA literature (BMA,1999), was completed reasonably well among the set of EISs. For the most part, various hazards were discussed for construction, operation and decommissioning of the nuclear reactors. A significant omission, however, was detected in the Bruce A and Pickering A Return to Service EISs: there was no mention of asbestos exposure during the decommissioning stage (OPG, 2000b; BP, 2002). Large amounts of asbestos, a known carcinogen, can be released during dismantling activities (Dodic-Fikfak et al., 1999). Nevertheless, barring this exception, a comprehensive range of hazards was successfully identified in the EISs.

Finally, each EIS incorporated an explicit discussion on the types and quantities of wastes that would be produced in the distinct phases of a nuclear power plant. The subject of radioactive waste disposal is central to any arguments countering renewed interest in nuclear power (Ansolabehere et al., 2003). Therefore, a clear discussion on the options of waste management would be required in any nuclear power EIS. Although there is no existing long-term facility for radioactive waste in Canada, the proponents identified OPG's Western Waste Management Facility to handle wastes for the time being (OPG, 2007d; BP, 2008c; OPG, 2009d). For wastes produced in the 
future, confidence has been allotted to the Nuclear Waste Management Organization, made possible by the Nuclear Waste Fuel Act of 2002 (OPG, 2007c). This organization has been entrusted with recommending a long-term waste strategy to the Canadian government. The treatment of waste was satisfactory for the purposes of the hazard identification phase. However, this topic will be be revisited in a later discussion on sustainability.

\subsubsection{Hazard Identification Weaknesses}

The PRA criteria dominated the shortcomings found in the hazard identification stage. But prior to an investigation of this matter, another issue deserves comment. The HRA literature repeatedly argues that a probabilistic approach to any parameter in a risk assessment is far superior to a point estimate calculation (Finley et al., 1994; von Stackelberg and Burmaster, 1994; Paustenbach, 2000). Although any given parameter may be represented by a probabilistic distribution, this does not mean that every parameter must be included in this manner; only the important values can be determined probabilistically (Finley et al., 1994). This point relates to radionuclide emissions from a nuclear reactor, which can fluctuate over time. Tritium emissions, due to the nature of increased neutron activation within the reactor core, rise over the course of a CANDU lifetime (Galeriu et al., 2003). These increasing emissions present an appropriate opportunity to apply probabilistic distributions, in order to represent the potential range of possible emission values. Unfortunately, this method was not adopted and instead, average point estimates were used. Instead of quantitatively assessing tritium accumulation, the EISs alternatively stated that heavy water from each 
plant is transported to the Tritium Removal Facility, located at the Darlington site. The effectiveness of this practice for reducing tritium over time, however, has been put into question (Fairlie, 2007).

During the derivation of the best practice framework, the importance of PRA was examined and then integrated into each section, where appropriate. Unfortunately, the use of PRA to evaluate accident scenarios appeared to be the only PRA criterion in the hazard identification section that was satisfied. The credibility of the PRA component varied considerably among each project. Two of the EISs relied on PRAs that assessed a different technology than the reactor in question. The first example was the Bruce A Restart project, which used the Bruce B Risk Assessment (BBRA) to estimate the source terms during accident scenarios (BP, 2002). The BBRA was used because the Bruce A Risk Assessment (BARA) was not available at the time of the EA. Justification is given: "because of their similarity, the BBRA is considered to be a reasonable approximation of the risk associated with the restart of Bruce A" (BP, 2002: 5-102). The extreme sensitivity of PRA challenges the merit of this substitution. To illustrate, an American nuclear plant operator, Entergy, calculated external and internal events to produce PRAs for their nearly identical Indian Point (IP) 2 and IP3 plants. The core damage frequencies produced were 1.4 per 10000 reactor-years for IP2 and 0.9 per 10 000 reactor-years for IP3 (Thompson, 2008b). These values are intriguing when compared to BP's safety limit for severe core damage at the time of the Bruce A Restart: 1 in 10000 (BP, 2002: 5-106). This example demonstrates that if these two identical plants were subjected to this safety limit, only IP3 would be deemed acceptable. Nevertheless, BP outlined 12 differences between the two plants; it cannot be 
determined how these differences would affect the outcome of a PRA (BP, 2002: 5-103). Suspiciously, the Bruce A reactors predate the Bruce B reactors by seven years, and therefore, would experience more age-related emission characteristics (BP, 2005a).

The second EIS that relied on a different design was the Bruce New Build. Although the routine emissions for the new Bruce reactor were modeled using real values derived from the proposed reactors, a review of Appendix F of the Radioactivity TSD reveals that accident scenario source terms were based on the core inventory of the Bruce $\mathrm{B}$ reactor (BP, 2008b: $\mathrm{F}-8)$. This is problematic, as the older CANDU reactor design at the Bruce B plant is not one of the proposed reactor technologies for the Bruce New Build, thus providing an inappropriate inventory from which to base accident scenarios. These two examples represent non-trivial substitutions; discrepancies in age and technological design can markedly influence reactor emission characteristics and accident risks (Lochbaum, 2000).

The importance of including both internal and external events in PRA has been stressed by numerous authors (USNRC, 1990; Sues et al., 1990; Thompson, 2008b; NEA, 2009). While internal events were examined, there was little consideration of external events or malevolent acts in the PRAs for each plant. For instance, the Pickering A Risk Assessment (PARA), BBRA and BARA each excluded an analysis of external events (OPG, 2000b; BP, 2002; BP, 2005a). When initially released, the Pickering B Risk Assessment (PBRA) also excluded external events; however, the CNSC subsequently reviewed the PBRA and recommended some substantial revisions be made for initiating events and uncertainty (Thompson, 2008a). A summary report of the PBRA methodology was released in November of 2008 and still provided no 
evidence of external event consideration (OPG, 2008). As PRAs are not willingly released to the public, the researcher was unable to ascertain whether OPG ever actually made the required changes to the PBRA. Finally, the Bruce and Darlington New Build projects used the PRAs for each reactor that were provided by the vendors. The Darlington Malfunctions, Accidents and Malevolent Acts TSD states that the ACR-1000 and AP-1000 PRAs consider both internal and external events, but the PRA for the USEPR only accounts for internal events (OPG, 2009c). It is also noted that each PRA is a Level 1 analysis, with only some Level 2 components. Ultimately, the exclusion of external events from a PRA would serve to underestimate the risk of catastrophic failure from a nuclear power plant.

The discussion thus far has focused on methodological weak points, but now attention is turned to PRA results. The PARA listed seven ex-plant release categories (EPRC) with corresponding source term estimates. Each category constitutes a group of accident sequences that result in similar environmental releases. EPRC1 and EPRC2 represent the largest release categories, which were also the only categories that excluded an accompanying estimation of the source term (this particular omission is unsettling, as most public concern would be over the largest releases, not the smallest ones) (OPG, 2000b). Fortunately, this same omission was not repeated in the BBRA, BARA, or the PBRA, as source terms for each release category were provided (BP, 2002; BP, 2005a; OPG, 2007a). The same cannot be said for the Bruce and Darlington New Builds. The Darlington EIS represented a marginal improvement from the Bruce EIS, which erroneously relied on an outdated CANDU design: "a core radionuclide inventory was selected from the three reactor technologies based on 
factors such as maximum reactor core size, maximum fuel burnup rate and use of (relatively) high enrichment fuel" (OPG, 2009c: 4-50). Nevertheless, the source terms provided are mostly based on the small and large release values, as outlined by the RD-337 and are "not necessarily characteristic of all potential releases for the reactor technologies" (OPG, 2009c: 4-51). For reference, the "large release" value of Cs-137 that is defined by RD-337 constitutes only $0.1 \%$ of the quantity of that particular radionuclide released in the Chernobyl accident (BP, 2008c: 6-70). Furthermore, the use of such bounding estimates to assess accident scenarios have long been criticized in other jurisdictions (Mueller, 1996). Therefore, both new build projects present unreliable bases of accident source terms, biasing any subsequent analyses of potential population exposures.

\subsection{Exposure Assessment}

The core of the exposure assessment step is to take the quantities of each hazard, identified in the previous step, and establish human exposures via environmental modeling. A robust exposure assessment in an EA would consider all of the important human exposure pathways. This section was arguably the best performed, half of the EAs receiving an "A". Table 4.2 displays the results of the exposure assessment evaluation (see Table 3.2 for a summary of the best practice criteria). 


\begin{tabular}{|l|c|}
\hline \multicolumn{1}{|c|}{ EA } & $\begin{array}{c}\text { Exposure } \\
\text { Assessment Results }\end{array}$ \\
\hline Pickering A Restart & B \\
& $17 / 22$ \\
\hline Bruce A Restart & B \\
\hline Bruce A Refurbishment & $17 / 22$ \\
\hline Pickering B Refurbishment & B \\
& A \\
\hline New Bruce & $18 / 22$ \\
\hline New Darlington & A \\
& $18 / 22$ \\
\hline
\end{tabular}

Table 4.2. The results of the Exposure Assessment section.

\subsubsection{Exposure Assessment Strengths}

Most of the best practice criteria for the exposure assessment were adequately met. Several parameters, key to this step, were initially defined in the EISs. Three distinct study areas were established in each EA to identify populations at varying exposure levels: site (located within the $914 \mathrm{~m}$ exclusion zone), local (within $10 \mathrm{~km}$ ), and regional (extending beyond local, but varying among the EAs). In addition, temporal boundaries were extended to the end of the decommissioning phase of each plant (OPG, 2000b; BP, 2002; OPG, 2009d). The EISs also incorporated detailed descriptions of the facility site. A survey of the unique geographic features of the plant's location would contribute to a sound examination of the dominant human exposure pathways. 
Prior to calculating the additional exposures that are caused by the restart, extension or construction of a new reactor, it is imperative to establish baselines for the more important radionuclides. Each site had existing nuclear reactors and therefore, had the benefit of previous monitoring efforts that extended over several decades. Even the most recent plant, Darlington, contained monitoring data dating back to the 1970s (OPG, 2009f). Monitoring activities, in collaboration with Health Canada, focused specifically on tritium, Carbon-14, noble gases, iodine, as well as gross beta and gamma radiation. Radiation of this nature was measured in gaseous and liquid emissions, water samples, precipitation, milk from local farmers, fish, garden vegetables and other aquatic and terrestrial media (OPG, 2000c; BP, 2002; OPG, 2009d). Also, dosimetry is practiced with workers, who are reportedly monitored for all occupational doses. These data were used to assist and validate proposed environmental models of future exposures. Furthermore, the more recent EAs used information from earlier assessments to justify their methods and assumptions. Completed EAs contain a wealth of information, but are seldom used in subsequent EA studies. Additional sources of radiation were examined, including natural background and medical exposures. A point worthy of attention here is the case of the Darlington New Build. All of the existing reactors in Canada are of the CANDU design and consequently produce a similar quality and quantity of radionuclides. This is not so with the new build projects, as technologies other than CANDU are being considered. Different technologies present different core inventories and consequently, different radioactive emissions. The Darlington EA conducted additional monitoring activities to establish baseline levels of radionuclides associated with the proposed technologies (OPG, 2009f). The studies 
of the Bruce EA, conversely, did not examine radionuclides other than those normally associated with CANDU technology.

Conservative assumptions were applied in the environmental monitoring and modeling data to quantify human exposures: a hypothetical person obtained all of his 62 vegetables and fish from the area surrounding the plant. It is also commendable, particularly when dealing with ionizing radiation, that infants, children and adults were assessed separately as the critical groups in each HRA. Infants are much more sensitive to the effects of ionizing radiation; solely assessing the exposure to adults would be insufficient. To further verify the habits of the different critical groups, surveys were conducted in 1997 to ascertain any distinctive habits of groups in the surrounding area $(B P, 2005 a)$.

In addition to the assessment of the environmental transport of radionuclides, non-radioactive chemicals were also considered. Maximum emission values, mostly for boiler discharges, were entered and modeled using atmospheric dispersion data.

\subsubsection{Exposure Assessment Weaknesses}

Despite the good efforts that were evident in the exposure assessment stage, some deficiencies did remain. Although gamma and beta radiation were accounted for during monitoring activities, there was little mention of alpha radiation. In fact, the Darlington and Pickering B Refurbishment EISs were the only studies to mention gross alpha radioactivity in reactor emissions (OPG, 2007d; OPG, 2009e). However, there is still no evidence of monitoring this type of radioactivity in the environment in either EIS.

\footnotetext{
62 "His" is intentionally used here, since HRAs, including the examples in this research, make use of the reference man; the legitimacy of this practice is questioned in subsection 4.3.2.
} 
This is alarming as "the radiation weighting factor for...alpha radiation is 20 ", meaning the harmful effects of alpha radiation in humans are 20 times those of gamma or beta radiation 63 (OPG, 2007c: 2-2).

It is very apparent in the HRA literature that probabilistic methods, employing Monte Carlo techniques, are a substantial improvement on single point estimates (Paustenbach, 2000). Similar to the case of tritium in the hazard identification section, all exposure values in the reviewed EISs were single values as opposed to probability distributions. Workers are exposed to much higher and more variable doses of ionizing radiation than the public; much higher exposures can occur during refurbishment and decommissioning activities than under normal operation conditions, since unexpected situations can compromise safety barriers (Dodic-Fikfak et al., 1999). The range of different exposure levels to which workers are subjected constitute an ideal situation to employ a probabilistic approach. Rather than averaging the different values, Monte Carlo techniques would have provided a range of exposure scenarios and ultimately, a more accurate assessment of health risk.

Although the findings of the exposure assessment indicate this component to be well done, as illustrated in Table 4.2, these results may be somewhat inflated. In terms of baseline environmental data, the monitoring programs in place at each facility are extensive. One issue, however, that received comparatively less attention was the baseline health information of exposed populations. These data are considered to be fundamental to the undertaking of any HIA (Parry and Kemm, 2005) and essential to

\footnotetext{
63 The seriousness of this omission was recently made apparent by an alpha radiation leak in November of 2009 during Bruce A refurbishment activities. This incident is under current investigation, but so far nearly 200 workers are thought to have been exposed to this type of radiation (Bruce Power gives prelim alpha radiation report to CNSC, 2010).
} 
validate any impact predictions (Petticrew et al., 2007). None of the restart or refurbishment EISs attempted to include specific background health information (OPG, 2000b; BP, 2002; BP, 2005a; OPG, 2007d). However, the Pickering and Darllington EISs alluded to the Radiation and Health in Durham Region study, which states cancer rates in that region appear normal64. It appears the only EIS to offer community health data was the Bruce New Build. Various health related statistics (cancer data excluded) are given for the Grey Bruce Health Unit, as well as information on the health care resources of the area (BP, 2008a). The Darlington EIS purports to provide similar information, but an examination of the "community health profiles" component of the Human Health TSD reveals little, if any, health related data on Clarington (OPG, 2009b). Without this key information, any adverse health impacts from a project would certainly go unnoticed 65 .

One final matter absent in the exposure assessment was an analysis of the temporal extent of released radionuclides in the environment. Although radioactive releases would cease with the conclusion of normal operations, some radionuclides have extremely long half-lives (CERRIE, 2004) and would consequently continue to remain hazardous for many years following decommissioning. Not in the assessment of normal operations or large-scale releases was there any indication of the persistence of some of the more long-lasting radionuclides 66 ; although the Pickering B Accident TSD

\footnotetext{
${ }^{64}$ Aspects of this study have been questioned, not the least of which was its initiation in 1983,12 years after the Pickering facility came online (Bertell, 2007).

65 Indeed, no monitoring of health indicators in the exposed population was proposed in any EIS; however, see subsection 4.5.1 for a discussion on a Cancer Care Ontario study, whose objective is to detect cancer patterns around nuclear power plants in Durham Region.

${ }^{66}$ Assessing the risks to future generations would be unwarranted; rather, supplying a temporal estimation of the environmental radiation would suffice.
} 
did provide a table of some half-lives for some of the more dominant radionuclides (OPG, 2007a).

\subsection{Dose-response Assessment}

The information gained during the exposure assessment studies is then analyzed in the dose-response assessment. In stark contrast to the previous HRA step, the doseresponse assessment was undoubtedly the worst performed. The results in Table 4.3 attest to this statement (see Table 3.3 for a summary of the Dose-response criteria).

\begin{tabular}{|l|c|}
\hline \multicolumn{1}{|c|}{ EA } & \multicolumn{1}{|c|}{$\begin{array}{c}\text { Dose-Response } \\
\text { Assessment }\end{array}$} \\
\hline Pickering A Restart & $\mathbf{C}$ \\
& $9 / 15$ \\
\hline Bruce A Restart & $\mathbf{C}$ \\
\hline Bruce A Refurbishment & $9 / 15$ \\
\hline Pickering B Refurbishment & $\mathbf{F}$ \\
& $6 / 15$ \\
\hline New Bruce & $\mathbf{C}$ \\
& $10 / 15$ \\
\hline New Darlington & F \\
& $5 / 15$ \\
\hline
\end{tabular}

Table 4.3. The results of the Dose-response Assessment.

\subsubsection{Dose-response Assessment Strengths}

In spite of the limitations in this section, some best practice criteria were satisfied consistently in the EISs. For example, each EIS contained dose-response data from 
the ICRP, as well as additional information on ionizing radiation from such recognized authorities as UNSCEAR and the NAS. Each EIS ${ }^{67}$ accepted the LNT model, which assumes risks increase proportionally for every exposure, to estimate risks from ionizing radiation. The LNT model is presumably a precautionary approach to radiation protection. The ICRP, as well as other international bodies that review the literature on low levels of ionizing radiation, have confirmed the LNT approach, asserting that no other theory presents enough evidence to void this model (CERRIE, 2004).

Coincident with the exposure assessment of different critical groups, doses to hypothetically exposed individuals of varying ages were present in each EIS. Similar to exposure assessment values (e.g., inhalation rates), DCFs are age-dependent (ICRP, 2006). This distinction is important as it would be erroneous to assign the same risk levels to infants as adults; damage that occurs in infants has a much greater period of time to develop into a health detriment than damage in adults (Busby and Fucic, 2006).

Additionally, the EA studies found hydrazine to be the most threatening chemical to human health and warranted a specific HRA in most cases (OPG, 2000b; BP, 2002; BP, 2005a; OPG, 2009d). Relatively high amounts of hydrazine were predicted to be released during routine operations; nevertheless, these analyses found the exposures to be within the toxicity reference values.

\subsubsection{Dose-response Assessment Weaknesses}

Continuing the final thought of the dose-response assessment strengths, the Bruce new build was the only EA that failed to analyze the exposure of hydrazine. As a

\footnotetext{
67 The Bruce New Build EIS did recognize the LNT to predict risk estimates, but then diverged from the other EAs and embraced the hormesis theory. This deviation is elaborated upon in subsection 4.4.2.
} 
recognized carcinogen that is emitted via blowdown from the coolant towers, the other EAs conducted specific HRAs on hydrazine to evaluate the potential toxicity to humans. This EIS acknowledged that "the most toxic chemical released to the environment is hydrazine" with regard to spills, yet there was no justification for the absence of a formal consideration of health risk (BP, 2008c: 6-11).

The most prominent shortcoming of the dose-response assessment was a limited examination of the health effects of ionizing radiation at both acute and chronic levels. To begin, in spite of the fact that the EISs generally presented a reasonable illustration of the possible health detriment attributed to ionizing radiation, some deficiencies were evident. Remarkably, the Bruce A Refurbishment contained no section devoted to human health in the main document, nor did it include an appendix or separate TSD of this subject; this was an exception to the other EISs (BP, 2005a). Admittedly, the researcher was not able to obtain the Radiation and Radioactivity TSD, but this particular TSD reviewed with the other EISs did not incorporate a review of the health effects from ionizing radiation, so it is doubtful that the Bruce A Refurbishment EIS would be an anomaly in this respect. Likewise, though much more subtle than the previous deficiency, the Bruce New Build EIS failed to provide a thorough account of the various health impacts of ionizing radiation. First, this document claims that certain cancers are not induced by radiation, including lymphomas and prostate cancers (BP, 2008a: 59). While the developments of certain cancers are particularly radio-sensitive, knowledge of causal associations of other cancers is constantly being updated. For example, Hodgkin's and non-Hodgkin's lymphomas have been an area of uncertainty, but recent research suggests an association with ionizing radiation (Richardson et al., 
2009). As well, studies in the past have identified an increased risk of prostate cancer with higher exposures (Rooney et al., 199368; Ritz et al., 2000) and the NAS's BEIR-VII report outlines increasing risk levels of this cancer with higher exposure levels (NAS, 2006: 312). It appears that the term "radiation-induced" has been confused with "radiosensitive" in this instance, i.e., although some cancers are more sensitive than others, "radiation-induced lowered immune responses can affect tumors at many sites within a few years of immunosuppression" (Wing et al., 1997: 54).

Second, the same document offers the following unsupported claim:

To date, ionizing radiation at any dose has not been identified as a cause of hereditary effects in humans. No evidence of a statistically significant increase in genetic defects has been observed in any group of humans, including the children of the atomic bomb survivors and the Chernobyl accident (BP, 2008a: 59).

The first statement is erroneous, depending on the subscribed definition of "hereditary effects", and the second statement is misleading. For example, Dubrova et al. (2002) studied several generations of families exposed to nuclear weapons testing in Kazakhstan and concluded that radioactive fallout "roughly doubled [the] germline mutation rate in the affected population" (Dubrova et al., 2002:1037). Similarly, another study also found significantly higher mutation rates in offspring of exposed individuals from a radiological accident involving Cs-137 in Goia, Brazil, when compared to offspring of unexposed individuals in the same region (da Cruz et al., 2008). The fact

\footnotetext{
68 This particular study found an increase in prostate cancer two orders of magnitude higher than expected.
} 
that these mutations ${ }^{69}$ did not correspond with genetic defects is immaterial: these studies demonstrate the hereditary nature of radiation insult to humans.

In addition to describing the physical health effects of radiation, the HIA literature maintains the importance of including any studies that concern the exposed population or specific technology (Health Canada, 2004c). Several epidemiological studies have been done, mostly on the population in the vicinity of the Pickering station, and were mentioned in most of the EISs. The exception was the Bruce A Refurbishment, which was the same EIS that did not include a coherent section on human health. This particular EIS, as well as the Bruce New Build, also omitted a subsequent discussion of the limitations of the findings of these studies.

Only one EIS, the Pickering B Refurbishment, attempted to differentiate between internal and external radiation ${ }^{70}$; collective doses to workers were provided separately in terms of external and internal doses (OPG, 2007c:4-103). Once again, only this EIS and the Darlington New Build EIS offered an explanation of differences between alpha, beta and gamma radiation (OPG, 2007c; OPG, 2009e). Furthermore, none of the EISs discussed possible bioaccumulation of certain radionuclides in certain parts of the body, nor was there any mention of possible synergistic relationships between ionizing radiation and other materials. This second matter is relevant to the decommissioning phase of nuclear power plants, where asbestos exposure is a likely scenario for workers; the detriment caused by this material may be exacerbated by suspected synergistic interactions with ionizing radiation (UNSCEAR, 2000). A wealth of

\footnotetext{
69 Many gene mutations do lead to inherited diseases; therefore, the study of radiation-induced changes is essential to understanding the genetic risks of human exposure to ionizing radiation (Dubrova, 2003).

70 Other EISs did define internal and external radiation; however, the Pickering $B$

Refurbishment was the sole document to differentiate between internal and external doses.
} 
information exists on the important distinctions between different types of exposures (external/internal) involving different types of radiation (alpha/beta/gamma), yet, for the most part, the different capacities of ionizing radiation types were lumped together in these EISs.

None of the EISs accounted for differences in radio-sensitivities of males and females; instead, the differences in sensitivities between the sexes were averaged. The characteristics of the "reference man" were used for DCFs and cancer risks (BP, 2008b). The NAS's BEIR-VII provides evidence for the higher cancer risks of females than males for the same exposure level (NAS, 2006). Therefore, the methodology used for dose conversions would understate the effects of radiation to women.

The off-site health consequences of accident scenarios predicted by a Level 3 PRA fall within the rubric of dose-response assessment. This area was particularly poor, as the EISs only supplied maximum individual and population doses without actually translating these doses into individual/population health impacts. Instead, the impacts to the public from an accident were expressed as the probability of a delayed fatality, taking into consideration the calculated low probability of the accident occurring (OPG, 2000b; Bruce A, 2002; BP, 2005a; OPG, 2007d). Merging these two values obfuscates the possible consequences of an accident and does little to inform the public of the risks involved in reactor accidents. One interviewee articulated his disapproval of this practice:

It is not correct to go through a PRA for an accident and take that probability and incorporate that into the HRA...Once that event occurs, the probability is one, then you go through the health risk analysis. You cannot merge the two...they don't have a meaningful number at the end (Interviewee \#4 per. comm., 2009). 
This practice is contrary to previous studies in which "offsite consequences" have been defined as "the number of early fatalities in the area surrounding the site" [italics added] (USNRC, 1990:2-3). Furthermore, the fallout of large quantities of radiation can render surrounding land uninhabitable for a period of time, yet this information was also mostly absent. The Darlington and Bruce New Build EISs did mention the possibility of population relocation, but estimates were based on release parameters outlined in the CNSC regulatory document RD-337, and not on PRA-generated source terms (BP, 2008c; OPG, 2009c). The exclusion of these features preclude all of the PRAs from being Level 3 assessments (Zandvoort, 2008). Without all of this information, the range of health risks of possible accidents, especially those involving severe releases, remain largely unknown.

\subsection{Risk Characterization}

The risk characterization step is the final step of HRA. All of the information and data gathered during the process are now collated to communicate the incremental risk that the project contributes. To accomplish this effectively, the definition of an acceptable risk must be clearly established. Furthermore, the confidence of the calculated risks must be indicated through a discussion of uncertainty. Finally, this is an opportune area to confirm monitoring and mitigation activities, in light of the potential project risks. The results of this evaluation section are mixed, illustrated by Table 4.4 (see Table 3.4 to review the best practice criteria for this section). 


\begin{tabular}{|l|c|}
\hline \multicolumn{1}{|c|}{ EA } & $\begin{array}{c}\text { Risk } \\
\text { Characterization }\end{array}$ \\
\hline Pickering A Restart & C \\
& $13 / 19$ \\
\hline Bruce A Restart & D \\
\hline Bruce A Refurbishment & $11 / 19$ \\
\hline Pickering B Refurbishment & C \\
& $12 / 19$ \\
\hline New Bruce & B \\
& $15 / 19$ \\
\hline New Darlington & C \\
& $13 / 19$ \\
\hline
\end{tabular}

Table 4.4. The results of the Risk Characterization section.

\subsubsection{Risk Characterization Strengths}

Regulatory documents from the CNSC outline what qualifies as an acceptable risk, in terms of both acute and chronic exposures. The internationally accepted standard for chronic public exposure to anthropogenic radiation is $1 \mathrm{mSv}$ per year, compliance with which is demonstrated via HRA. The CNSC regulatory document, RD-337, provides acute risk limits: the sum of core damage frequencies must be below 1 in 100000 reactor-years, the frequency of a small scale release (more than $10^{15} \mathrm{~Bq}$ of I-131) must be less than 1 per 100000 reactor-years and the frequency of a large-scale release (more than $10^{14} \mathrm{~Bq}$ of $\mathrm{Cs}$-137) must be below 1 in a million reactor-years (CNSC, 2008c). Regrettably, adherence to these criteria were not followed as diligently as the chronic exposure limits and is described in detail in subsection 4.4.2. 
As previously mentioned in the exposure assessment analysis, all of the sites have existing nuclear reactors and consequently, already have detailed monitoring programs ${ }^{71}$. Dosimetry is practiced on workers to ensure exposure levels stay within permissible doses and many components of the environment are monitored for radiation levels. Without divulging much detail on safety procedures, the ALARA approach is advocated in order to minimize worker and public exposures.

\subsubsection{Risk Characterization Weaknesses}

Prior to the release of RD-337 by the CNSC in 2008, OPG and BP abided by their own safety goals, which were synonymous, for the various findings of a PRA (BP, 2005a: 3-108; OPG, 2007d: 7-30). These guidelines specified that the risk target for severe core damage was 1 in 100 000, but the limit was 1 in 10000 per reactor year; the difference is that "the safety goal limit represents the limit of tolerability of risk above which action should be taken to reduce risk" and "the safety goal target represents an objective toward which the 'facility should strive"' (OPG, 2007d: 7-30). The CNSC did provide its own guidance pertaining to PRA in 2005, but this document did not outline official safety goals that a PRA must achieve (CNSC, 2005b). Thus, the Bruce and Darlington New Build projects subscribe to the limits stated in RD-337 (described in the previous subsection), and all prior EAs followed the OPG and BP safety criteria. The Pickering A Restart EA applied the PARA, which was completed in 1995, to judge potential accident risks of the restart project. OPG claimed that over $\$ 1$ billion would be spent on repairs and improvements for the plant, but did not state how this would affect

\footnotetext{
71 Please see the subsection 4.2.1 for details on monitoring efforts.
} 
the results of the PARA. Furthermore, the PARA found the risk of severe core damage to be 1.3 in 10000 , thus breaching OPG's own safety limit. OPG acknowledged this deficiency and asserted it would make the appropriate changes to lower the risk to acceptable levels; however, there was no discernible plan as to how this would be accomplished (OPG, 2000b).

Similarly, the Bruce A Refurbishment project based accident scenario information on the conclusions of the BAPRA. The risk for a large release must be kept below 1 in 100000 , yet the BAPRA found three release scenarios that entailed higher frequencies: 1 in 38 000, 1 in 36000 and 1 in 33000 reactor years (BP, 2005a). Bruce Power affirmed that these findings are reasonable, since they fall below the 1 in 1000 frequency of "dual failure" accidents, as required by the CNSC. Apparently, the importance of the dual failure accident criterion voids the BAPRA findings of large scale release frequencies. This spurious reasoning is not surprising, as at that time "there [were] no regulatory limits for the accidents identified in the probabilistic risk assessment" (BP, 2005a: 3-108).

Aside from the typical consequences predicted in a full Level 3 PRA (early fatalities, cancers, evacuation of contaminated lands), nuclear accidents also have the potential to cause serious psychological damage. Hartsough and Savitsky (1984) documented an assortment of psychological impacts from the TMI accident, including sleep disturbances, fear, anxiety, and psychosomatic symptoms, among others. Similarly, mental health effects were extensively documented in the aftermath of the Chernobyl meltdown, many of which were compounded by the forced evacuations (Fairlie and Sumner, 2006). Baverstock and Williams (2006) asserted that the stress 
after the Chernobyl accident stemmed from fears over exposure to an invisible, but highly dangerous agent; worries over children's health; and mistrust of the information provided by authorities. These authors further posited that despite the nuclear industry's views on the irrationality of these specific impacts, they do represent a significant public health concern. Recently, psychological stress has even been suggested as a mechanism to increase ionizing radiation's capacity to cause DNA damage (Cwikel et al., 2010). Despite the potential for serious psychological implications, only two EISs, the Pickering B Refurbishment and the Darlington New Build, attempted any mention of such consequences from a nuclear accident (OPG, 2007a; OPG, 2009d).

Possibly the greatest disparity in the risk characterization section was the lack of a consistent uncertainty analysis, in both HRA and PRA. Although none of the referenced PRAs were available for review, an indication of uncertainty was not given with the PRA accident frequency values in the EISs. However, as mentioned previously, the summary of the PBRA that was released for the public did incorporate some element of uncertainty considerations (OPG, 2008), but this was the exception. NUREG-1150, regarded as the high point of PRA (Thompson, 2000), featured severe core damage and release values as probabilistic distributions, to obtain a better grasp of the confidence in the resulting frequencies (USNRC, 1990). Unfortunately, none of the nuclear accident sections in the EISs adopted this approach. The HRA situation represented a marginal improvement from that of PRA: most EISs addressed uncertainty by using conservative values, wherever possible, and underscoring the importance of follow-up to validate EA predictions. Unfortunately, as discussed 
previously, no EA took a probabilistic approach to HRA, regarded as the superior method for dealing with uncertainties (von Stackelberg and Burmaster, 1994). The Bruce A Refurbishment explicitly discussed uncertainty for the HRA of hydrazine and carried out a sensitivity analysis to determine if public doses would vary according to lifestyle activities (BP, 2005b). Appendix O of the Darlington Radiation Effects TSD (OPG, 2009e) also clearly assessed the sensitivities of several of the models and assumptions used throughout the EA. Though it was clear the EAs strived to take a conservative approach, the uncertainty of calculations ought to be highlighted.

One of the pillars of EA, and arguably the dominant approach to addressing uncertainty, is the Precautionary Principle. In spite of CEAA maintaining that projects "[be] considered in a careful and precautionary manner" (CEAA, s.4 (1a)), only the Bruce and Darlington New Builds clearly defined and claimed adherence to a precautionary approach (BP, 2008c; OPG, 2009d). While it is important to be in agreement with this concept, it is necessary to state exactly how it has been followed. One EIS clearly demonstrated a breach of the Precautionary Principle, which is ironic, as it was one of the EISs to define this concept. The Bruce New Build EIS promoted the hormesis model of low doses of ionizing radiation. This theory has not been accepted by major international scientific commissions and is therefore, unjustifiably referenced in this EIS (NAS, 2006; UNSCEAR, 2006). As the theory claims health benefits in place of detriments, the Bruce EIS tried to sway the reader by attempting to undermine the potential effects of radiation exposure (BP, 2008a).

Finally, the assessment of risks in regard to sustainability was included in each EIS, but to varying degrees. The restart and refurbishment EISs mainly stated that 
projects had little or no effect on the depletion of uranium reserves, as there are many other nuclear power plants also extracting uranium (OPG, 2000b; BP, 2002; BP, 2005a; OPG, 2007d). Some EISs contended that the project would have a positive effect on sustainability, by providing "clean" energy in place of burning fossil fuels (BP, 2005a; OPG, 2007d). The two new build projects did not include an assessment on the use of non-renewable resources. Instead, these EISs, particularly Darlington, examined sustainability from a social and economic perspective, and reported that the project would entail a positive effect (BP, 2008d; OPG, 2009d). One of the most prominent critiques of nuclear power is the lack of a long-term waste storage plan (Ansolabehere et al., 2003). However, this issue was not investigated under the context of sustainability, though clearly representing a burden to future generations.

\subsection{Process}

The process section incorporates best practice indicators that were identified in the literature and/or interviews, but did not fit into the four traditional HRA steps. This section is heavily influenced from dominant concepts in the HIA literature, rather than HRA or PRA. A sound HIA process would demonstrate openness and transparency, and would ensure information is presented in an accessible fashion. With the exception of one EIS, this section was mostly satisfactory (see Table 4.5). The evaluation criteria for this section is shown in Table 3.5. 


\begin{tabular}{|l|c|}
\hline \multicolumn{1}{|c|}{ EA } & Process \\
\hline Pickering A Restart & A \\
& $13 / 16$ \\
\hline Bruce A Restart & C \\
\hline Bruce A Refurbishment & $11 / 16$ \\
\hline Pickering B Refurbishment & F \\
& B \\
\hline New Bruce & $13 / 17$ \\
& B \\
\hline New Darlington & $12 / 17$ \\
\hline
\end{tabular}

Table 4.5. The results of the Process section.

\subsubsection{Process Strengths}

The need to accept the WHO definition of the term "health" resounded throughout much of the HIA literature. Fortunately, with the exception of the Pickering A Restart, the EISs embraced the WHO approach to human health, which would ensure a more inclusive assessment (OPG, 2000b). At the time of the release of each EIS, it appeared that the project had not yet started, making it possible for findings of the HIA to potentially alter the project outcome. The EISs, for the most part, communicated concepts in clear language and with one exception, the Bruce A Refurbishment, included non-technical summaries of human health impacts (BP, 2005a). Units and terms were defined and used consistently throughout the the majority of the EISs. The inclusion of such summaries are essential for nuclear power EISs specifically, which can involve numerous volumes, appendices and TSDs. Often there is public concern over 
the potential human health impacts, so this information ought to be readily accessible without having to sift through large volumes of information.

A major pillar of the EA process is the public consultation process. All of the EISs outlined the efforts taken toward engaging the public. For instance, both proponents, OPG and BP, held public workshops to address various public concerns, and disseminated the results of these workshops to attendees and interested parties, as well as including this material on websites. Furthermore, each EIS also devoted a section on listing the most prominent public concerns, as identified in initial public meetings or submitted as public comments. These sections also detailed where such issues in the EIS could be found, or why a particular issue was omitted (OPG, 2000b; BP, 2002, BP, 2005a; OPG, 2007d; BP, 2008c; OPG, 2009d). These findings are congruent with a recent study by Bredimas and Nuttall (2008), who found the public consultation approach in Canada to be exemplary, when compared to six other nations utilizing nuclear power.

Though not directly funded by either of the proponents, the EAs for Darlington and the Pickering B Refurbishment benefited from an ongoing study by Cancer Care Ontario (OPG, 2007b; OPG, 2009b). According to the Human Health TSD for Darlington, this study is in its final stage and aims "to investigate cancer patterns and proximity of residence to nuclear reactors in Durham Region" (OPG, 2009b:4-49). The results of this study will hopefully improve public understanding and contribute to the knowledge of cancer patterns around nuclear power plants. 


\subsubsection{Process Weaknesses}

The most noticeable weakness evident in the six reviewed EISs, with regard to the process indicators, was the lack of a distinct human health chapter, appendix or TSD in the Bruce A Refurbishment EIS (BP, 2005a). The two EISs released prior to this project did include sections summarizing the salient impacts on human health, so it is surprising to see such an obvious omission.

The concept of peer review was highly regarded in both the literature review and the interview process. There are two separate entities which ought to be peer reviewed: the HRA, which is essentially the EIS itself, and the PRA, which is a separate document altogether. Full or partial reviews of the EIS document by other relevant FAs is standard practice under CEAA, but it is not a substitute for independent peer review. Only half of the EISs were subjected to such review: Pickering A Return to Service included a review by eight university professors from the University of Toronto and McMaster University; BP funded a review by the Saugeen Ojibway Nations and the Saguingue Metis Council for the New Build Project; and "independent specialists" conducted peer reviews on the technical studies and TSDs of the Darlington EA (OPG, 2000b; BP, 2008c; OPG, 2009c). The other EAs were just subjected to the standard FA review process (BP, 2002; BP, 2005a; OPG, 2007d). Still, the peer review process for the HRA was far better than that of PRA. In fact, contrary to past practices, PRAs are rarely independently reviewed anymore (Interviewee \#5 per. comm., 2009). Instead, the CNSC reviews PRAs; the public is no longer involved in the process. However, another document (Thompson, 2000) obtained by the researcher showed that some parts of the PARA had been reviewed by consultants at Acres International, but at that time it did not 
consider the numerous changes at the Pickering A plant post-1997. The Bruce A Restart EIS relied on the BBRA, which had been reviewed by the CNSC, but unfortunately is specific to the Bruce B plant ${ }^{72}$.

The situation is not much better for the Bruce A Refurbishment, although it did manage to employ the PRA for that particular facility. Regrettably, the EA screening was accepted on July 5, 2006 (CNSC, 2009b) and the CNSC only completed their review of the BAPRA in November 2006, concluding, "the review did identify some sources of uncertainty regarding the validity of the insights and their utility as input in the decisionmaking process" (CNSC, 2007a:15). Therefore, at the time of EA approval, the CNSC accepted unsubstantiated PRA results. The only circumstance in which the CNSC was able to review the plant-specific PRA and suggest changes prior to EA acceptance, was the Pickering B Refurbishment (CNSC, 2008a). The initial review concluded in August 2007, when the CNSC identified several methodological problems that needed to be remedied. The CNSC document that yielded this information also predicted the final review to be complete by January 2009, the precise time of EA approval (CNSC, 2009c). However, the researcher was unable to ascertain whether all of the CNSC recommendations had indeed been honoured. Nevertheless, it is still commendable that OPG released a summary of the PBRA methodology for public review. Finally, the two new build projects based much of the nuclear accident analysis on the generic vendor PRAs, none of which had been reviewed by the public or the CNSC.

Similar to the concept of independent peer review, the consultation of independent health experts was considered an important issue. As previously

\footnotetext{
72 The applicability of this document for Bruce A is questioned in subsection 4.1.2.
} 
mentioned, the EISs were subjected to review by other FAs, including Health Canada, but again, this is not a substitution for review by independent authorities. None of the EISs disclosed whether any independent health experts had been involved in the EA process.

A robust evacuation plan would detail various responsibilities and be influenced by the findings of a plant-specific Level 3 PRA (Zandvoort, 2008). This concept stems from recent efforts ${ }^{73}$, predominantly in the $E U$, to promote the use of PRA results for emergency plans; PRAs often provide detailed information that can be used to help implement accident mitigation measures (Kirchsteiger, 2006). Similar to the discrepancies in the PRAs for each plant, the extent to which the evacuation plan was outlined varied greatly among the EISs. In addition to lacking a distinct human health section, the Bruce A Refurbishment excluded substantial information on an evacuation plan (BP, 2005a). The two new build projects did delve into some detail on this matter, but the lack of a comprehensive PRA analysis for these projects limited the usefulness of such plans (BP, 2008c, OPG, 2009a). The Pickering B Refurbishment did partially achieve a substantive evacuation plan by integrating the doses from several accident scenarios with corresponding emergency procedures. However, measures during a worst case scenario were omitted from the discussion (OPG, 2007d).

The last point in the process section pertains to the composition of the panel in a panel review; therefore only the Bruce and Darlington New Build projects are relevant to this criterion. For screening and comprehensive study EAs, it is the RA who has control over the EA process and subsequent project approval. A panel review is supposed to

\footnotetext{
${ }^{73}$ Although research efforts in this area are more recent, the value of risk assessment for emergency response measures was recognized well over a decade ago (Canter, 1993).
} 
transfer this responsibility to a panel of three independent experts, who then make recommendations to the Minister of the Environment (Herring, 2005). The Minister subsequently provides public comments on the findings of the panel, at which point the RA must confirm a final project decision. Since the CNSC is always the RA for nuclear projects, one might expect the panel to consist of individuals other than members of the CNSC. Nevertheless, review panels for both Bruce and Darlington contain CNSC members, who even constitute the panel majority in the Bruce EA (CNSC, 2009d; CNSC, 2009e). Such situations call into question the purpose of seeking "independent" authorities to coordinate the EA process.

\subsection{Overall Evaluation of Best Practices}

The previous section examines the various criteria included in the best practice evaluation framework. A considerable amount of information has been presented throughout the five subsections combining HIA, HRA and PRA. This chapter now concludes with summaries of the HRA and PRA components. In essence, this section comments on the treatment of chronic and catastrophic risks in the nuclear power EISs.

\subsubsection{Health Risk Assessment}

The best practice evaluation framework proved to be successful at identifying strengths and weaknesses in the HIAs. The detailed analyses in the previous sections demonstrated the inconsistencies in the reviewed HRAs. Table 4.6 illustrates a comparison of the five components of the evaluation framework, as shown individually throughout the preceding sections. 


\begin{tabular}{|c|c|c|c|c|c|}
\hline EA & $\begin{array}{c}\text { Hazard } \\
\text { Identification }\end{array}$ & $\begin{array}{c}\text { Exposure } \\
\text { Assessment }\end{array}$ & $\begin{array}{l}\text { Dose-Resp. } \\
\text { Assessment }\end{array}$ & $\begin{array}{c}\text { Risk } \\
\text { Charac. }\end{array}$ & Process \\
\hline $\begin{array}{l}\text { Pickering A } \\
\text { Restart }\end{array}$ & $\underset{7 / 11}{\mathbf{C}}$ & $\begin{array}{c}\text { B } \\
17 / 22\end{array}$ & $\underset{9 / 15}{C}$ & $\underset{13 / 19}{\mathbf{C}}$ & $\underset{13 / 16}{\text { A }}$ \\
\hline $\begin{array}{l}\text { Bruce A } \\
\text { Restart }\end{array}$ & $\underset{7 / 11}{\mathbf{C}}$ & $\begin{array}{c}\text { B } \\
17 / 22\end{array}$ & $\underset{9 / 15}{C}$ & $\underset{11 / 19}{\text { D }}$ & $\underset{11 / 16}{\mathbf{C}}$ \\
\hline $\begin{array}{l}\text { Bruce A } \\
\text { Refurbishment }\end{array}$ & $\begin{array}{c}\text { B } \\
8 / 11\end{array}$ & $\underset{17 / 22}{\text { B }}$ & $\begin{array}{c}\mathbf{F} \\
6 / 15\end{array}$ & $\underset{12 / 19}{\mathbf{C}}$ & $\begin{array}{c}\mathbf{F} \\
8 / 17\end{array}$ \\
\hline $\begin{array}{l}\text { Pickering B } \\
\text { Refurbishment }\end{array}$ & $\begin{array}{c}\text { B } \\
8 / 11\end{array}$ & $\begin{array}{c}\text { A } \\
18 / 22\end{array}$ & $\underset{10 / 15}{\mathbf{C}}$ & $\begin{array}{c}\text { B } \\
14 / 19\end{array}$ & $\begin{array}{c}\text { B } \\
13 / 17\end{array}$ \\
\hline New Bruce & $\begin{array}{c}\text { B } \\
9 / 12\end{array}$ & $\begin{array}{c}\text { A } \\
18 / 22\end{array}$ & $\underset{5 / 15}{\mathbf{F}}$ & $\underset{13 / 19}{\mathbf{C}}$ & $\begin{array}{c}\text { B } \\
12 / 17\end{array}$ \\
\hline $\begin{array}{l}\text { New } \\
\text { Darlington }\end{array}$ & $\underset{7 / 12}{\mathbf{D}}$ & $\underset{18 / 22}{\text { A }}$ & $\underset{10 / 15}{C}$ & $\begin{array}{c}\text { B } \\
15 / 19\end{array}$ & $\begin{array}{c}\text { B } \\
13 / 17\end{array}$ \\
\hline
\end{tabular}

Table 4.6. The results of the five sections of the best practice framework.

Without question, the best performed area was the exposure assessment section. These findings can be attributed to the fact that each nuclear site has been established for several decades and has consequently produced large amounts of environmental monitoring data. These activities entail determining levels of radiation in such environmental media as air, water, fish and cow's milk. This information can be used to achieve baseline levels and help validate environmental modeling for future human exposures. Despite the extent of these monitoring practices, certain gaps remained; for example, discussions of alpha radiation were all but absent. Furthermore, the data were supplied as mean values, instead of taking advantage of probabilistic techniques; this approach can provide better estimates of the range of possible 
exposures (this is important for workers, who are subjected to much higher doses). Finally, EISs generally provided scarce information on the baseline health status of surrounding communities, something essential from which to understand future health impacts. This omission is not surprising, however, since direct efforts to ascertain future health data of surrounding populations were mostly excluded.

Most of the criteria not met in the hazard identification section pertained to PRA (discussed in the next subsection). Reasonably detailed descriptions of the reactor technology and the corresponding unique safety features were provided in the EIS, as well as examinations of waste management ${ }^{74}$. However, increasing reactor emissions throughout the lifetime of a nuclear plant were not considered. Additionally, the differences in emission values for different reactors was blurred in the new build EISs, which assessed a hybrid core inventory of the proposed reactor technologies.

Following these two steps was the dose-response assessment, certainly the poorest section encountered during the evaluation. Most (or all) of the EISs suffered from omitting various health effects of ionizing radiation, excluding DCFs, ignoring differences in internal and external radiation and failing to consider any synergistic interactions of ionizing radiation. Fortunately, the EISs adopted recommendations of the ICRP and (mostly) embraced the precautionary LNT model of low level radiation.

The final step, the risk characterization section, exhibited the largest range of results. The notion of an acceptable risk was defined, though not always honoured. As in the exposure assessment section, monitoring activities were promised to ensure the validation of risk predictions. The largest downfall of this component was the lack of

\footnotetext{
74 Note that no long-term disposal option has been agreed upon yet. The responsibility of a long term solution now rests with the Nuclear Waste Management Organization.
} 
consistent uncertainty analyses. A discussion on this matter is necessary to confirm the confidence of calculations, which unfortunately, was not always the case. Project risks were also assessed in the context of sustainability, whose definition ranged from concentrating on resource use in the earlier EISs (OPG, 2000b; BP, 2002) to maintaining social and economic matters in the more recent projects (BP, 2008c; OPG, 2009c).

The assessment of chronic health risks through HRA techniques was markedly better than the consideration of acute risks, as the next section will demonstrate. There does appear to be a slight trend toward improvement, as the better assessments, the Pickering B Refurbishment and Darlington New Build, were more recently completed. Nevertheless, as Table 4.6 captures, the overall achievement of HRA best practices proved to be quite fragmentary, save for the consistency of the exposure assessment section.

\subsubsection{Probabilistic Risk Assessment}

The best practice framework used the four main steps of an HRA, as well as an additional section for process indicators, to structure evaluation criteria. Best practices relevant to PRA were then integrated into the appropriate section. It would be useful to now distinguish this information to roughly determine the quality of the referenced PRAs. The subject of PRA has been addressed much throughout the previous section, so this analysis will be kept brief. Table 4.7 outlines the PRA-specific criteria found in 
the best practice framework ${ }^{75}$, with the results of collating these indicators shown in Table 4.8.

\section{PRA Criteria}

1.4. Discussion of accident scenarios by use of Probabilistic Risk Assessment (PRA) for each proposed technology?

1.4.1. Includes both internal (real reactor conditions) and external (e.g. tornadoes, airplane crashes) events?

1.4.1.1. Includes security/terrorism threats in external events?

1.4.2. Defines upper limit source terms for releases to the environment for all significant accident sequences?

3.4. Predicts magnitude of offsite health consequences to exposed population for all significant accident release categories (i.e. Level 3 PRA)?

4.1.4. Risks below Canadian/international/voluntary PRA standards with justification of any discrepancies?

5.5. Evidence of PRA independent peer review process?

5.5.1. Reviewed by individuals/groups not affiliated with the project?

5.12. Emergency Evacuation Plan guided by highest risk areas as outlined in full Level 3 PRA?

Table 4.7. The PRA-specific evaluation criteria.

\begin{tabular}{|l|c|}
\hline \multicolumn{1}{|c|}{ EA } & PRA Results \\
\hline Pickering A Restart & F \\
& $3 / 9$ \\
\hline Bruce A Restart & $\mathbf{F}$ \\
& $3 / 9$ \\
\hline Bruce A Refurb & $\mathbf{F}$ \\
& $2 / 9$ \\
\hline Pickering B Refurb & $\mathbf{D}$ \\
& $5 / 9$ \\
\hline New Bruce & $\mathbf{F}$ \\
& $2 / 9$ \\
\hline New Darlington & $\mathbf{F}$ \\
& $2 / 9$ \\
\hline
\end{tabular}

Table 4.8. The results from the PRA-specific evaluation criteria.

75 The references for each point have not been included in this chart; please see Appendix B for citations. 
It is clear to see Table 4.8 portrays a rather dismal picture of the quality and use of PRA in the reviewed EISs. The Pickering B Refurbishment achieved the highest mark of $5 / 9$, barely considered a passing grade. The reasons for these abysmal results are numerous.

First, including external events in PRA, though proven to contribute significantly to risk levels (USNRC, 1990), is not a routine practice in Canada. Indeed, a response of an OPG employee in a recent NEA survey confirmed that "no [external event] PSAs have been attempted at OPG" (NEA, 2009:59). The CNSC permits alternative means to assess external events (CNSC, 2005b). For example, instead of incorporating seismic events in the PRA, they are considered in a separate seismic margin assessment, which is regarded as less comprehensive (Thompson, 2000).

Second, security threats were included in some of the later EISs, but were not addressed by PRA. For instance, the Darlington EIS stated that OPG "completed a comprehensive review of the robustness of its existing nuclear assets against credible threats and accidents...including the consequence of aircraft strikes", yet there was no indication as to the credibility of this review, i.e., whether it was undertaken via formal PRA (OPG, 2009b:8-14). This is unfortunate, as Canadian reactors were not designed with the possibility of aircraft crashes in mind (BP, 2008c). Furthermore, this material is considered "prescribed information and cannot be released" (OPG, 2007d:10-56). This last comment captures the reluctance of nuclear power proponents to subject PRAs to peer review. More worrisome, however, is that possible radioactive releases from spent fuel ponds were not extensively analyzed; if terrorists were to successfully tamper with 
the cooling system of the pool, the spent fuel rods could ignite and potentially release more radiation than that of a core meltdown (Perrow, 2007).

Finally, upper limit source terms were rarely estimated; instead, there was a cavalier dismissal of such circumstances: "a Category 776 accident is impossible and is not considered further in the assessment" (BP, 2008c:6-33). The predicted impacts of a radionuclide release were isolated to some of the lower consequence events.

Predictions primarily identified population exposure levels, with minimal descriptions of resulting health effects and land contamination. Ultimately, this would provide little useful information for emergency plans in the event of a large-scale radiation release. The Pickering B Refurbishment earned its relatively high grade by publicly releasing information on the PBRA methodology, submitting a draft to the CNSC well before EA completion, and linking different accident scenarios with corresponding emergency measures; though there is still obvious room for improvement.

Using the evaluation criteria derived through this research, it is evident that the treatment of catastrophic health risks, through the use of PRA, represents a significant departure from best practices.

\subsection{Summary}

With the best practice framework outlined in Chapter 3, this chapter provided an in depth analysis of the EIS evaluations. Patterns of strengths and weaknesses in each of the five evaluation sections were described, addressing many concepts surrounding HIA, HRA and PRA. This chapter concluded with separate overall evaluations of HRA

\footnotetext{
76 The IAEA has suggested a nuclear accident classification scale, with Category 7 representing a "major accident".
} 
and PRA, which summarized the treatment of chronic and catastrophic risks in the reviewed EISs. 


\section{CHAPTER 5: CONCLUSIONS}

\subsection{Introduction}

The underlying purpose of this research is to determine the CNSC's commitment to best practices, by means of evaluating the consideration of chronic and catastrophic health risks in nuclear power plant EISs. This chapter briefly summarizes the methods undertaken to satisfy this research focus, in addition to the major findings from the HIA evaluations. Conclusions are reached on the overall compliance of the CNSC to best practices. Finally, any limitations of the study are addressed and future research opportunities proposed.

\subsection{Research Summary}

Until the passage of CEAA in 1995, nuclear power plants in Ontario avoided EA scrutiny. Since that time there have been several reactor restart, refurbishment and construction projects proposed, with some receiving approval. The CNSC is responsible for the regulation of nuclear power facilities in Canada and claims to use a risk-informed approach for all regulatory decisions (CNSC, 2005a). The research in this thesis strives to evaluate the consideration of human health risks in the HIAs for these projects, both chronic and catastrophic, through the use of a best practice framework. Four clear objectives were initially defined in Chapter 1 to achieve the main research goal.

The first objective was to establish best practices of HIA. A two-tiered approach was used to accomplish this task: first, a comprehensive literature review was conducted on the various methodologies of HIA, HRA and PRA, and second, the 
concepts gained from this review were used as the subject matter for an expert interview process. Upon approval of the study protocol by the Ryerson University Research Ethics Board, 15 authoritative sources on HRA and PRA, out of 60 contacted, were interviewed. The range of backgrounds of interviewees included epidemiologists, HRA/PRA practitioners, radiation consultants, nuclear regulators and low dose radiation researchers. The integration of prominent concepts present in the literature review and converging viewpoints identified in the interview process would be used to create the best practice framework.

The second research objective was satisfied by using this information to establish an evaluation framework. The framework was structured using the four traditional steps of HRA (hazard identification, exposure assessment, dose-response assessment, risk characterization), as well as adding a "process" section for criteria specific to HIA. A systematic discussion was undertaken to develop best practices for each section, concluding with a brief point-form summary of the derived criteria. Each criterion was only included if clear concordance was apparent among the reviewed literature and interviewed authorities.

To fulfill the third research objective, six nuclear power project EISs were selected for review with the derived framework. The selected assessments represented all of the return to service, refurbishment, and new reactor construction EAs in Ontario. The review protocol adopted similar methods of other EA researchers (Lee et al., 1999; Demidova and Cherp, 2005). One main difference, however, was that the framework adopted a binary approach. The reason for this was that a special focus on nuclear power made it possible to have much more specific indicators of best practice, which 
could either be satisfied or not. Additionally, the framework was also designed to be used as a possible scoping aid during the EA process.

Upon the completion of the EIS evaluations, it was possible to realize the fourth and final research objective: determining if the CNSC has ensured compliance with HIA best practices. As this objective coincides with the main research question, more detail is required than given for the previous objectives. Section 4.6 has already presented separate evaluations of HRA and PRA; therefore, the discussion in the next subsection reaches conclusions about the overall evaluation process.

\subsubsection{Compliance with Best Practices}

In Chapter 4, numerous examples of compliance and non-compliance documented throughout the reviewed EISs were illustrated, but here an important distinction must be made. Earlier it was noted that not all best practice criteria are of equal importance, and while adding up all satisfied criteria in each evaluation section can demonstrate general trends, identifying omissions of key indicators may be more revealing. It is here that the exclusion of critical HIA best practices are emphasized and used as justification to reject the reviewed EAs.

A study deploring the regulation of age-related risks in the US contended that instead of current practices, "sound, risk-informed decisions about the nation's nuclear power plants must be based on consistent, accurate risk assessment studies" (Lochbaum, 2004:18). Unfortunately, the same can be said of Canadian regulation, as the CNSC's failure to consider many non-trivial risks was abundantly evident in the reviewed EISs. The Pickering A Return to Service EA has been 
previously criticized for not calculating probabilities of the most serious accidents, finding an unacceptably high risk for severe core damage, not disclosing the $\$ 1$ billion plant upgrades to be made, and conducting the EA at the most basic (screening) level of assessment (Thompson, 2000; Standing Senate Committee on Energy, the Environment and Natural Resources, 2001). The Bruce A Return to Service EA is deemed deficient for relying on a different plant's PRA to assess catastrophic risks, if for nothing else. The successor to the Bruce A Return to Service, the Bruce A Refurbishment, was an even worse assessment. Contrary to all HIA literature, there was no cohesive section documenting the human health impacts, unlike all of the other reviewed EISs. Additionally, the significance of excluding monitoring efforts of alpha radiation was underscored last November when unanticipated alpha contamination caused nearly 200 workers to be sufficiently exposed for testing (Bruce Power gives prelim alpha radiation report to CNSC, 2010). The Pickering B Refurbishment, arguably the best EIS to be reviewed, also fell short of best practices: the PBRA excluded external events, which were also missing in all previous OPG risk assessments; offsite health consequences were reduced to the probability of early fatalities, while factoring in the probability of an accident actually occurring 77 ; and no indication of uncertainty accompanied the suspiciously low probabilities of the PBRA. Finally, the Bruce and Darlington New Builds can also be rejected. The Bruce EA advocated a hormetic doseresponse curve, declared the impossibility of a category 7 accident, and used the core inventory of a different plant to predict accident source terms. The Darlington EA, though representing a clear improvement on the Bruce New Build EA in many respects,

\footnotetext{
77 The low probability of an accident occurring would greatly dilute the predicted magnitude of offsite health consequences.
} 
failed to substantially characterize the health of the surrounding population; relied on a mix of vendor Level 1/2 PRAs and RD-337 safety goals to generate accident source terms, not actual PRA outputs; and did not substantially consider the risks of malevolent acts, particularly with regard to spent fuel storage pools. Although many of these deficiencies were common to most and in some cases, all EISs, enough were present to individually reject the EAs on separate grounds.

Nuclear power plants are high risk facilities; their irreducible complexity and unique potential for catastrophic accidents necessitate vigilant regulation to protect public health and safety. This thesis investigated the regulatory rigor of the CNSC by evaluating compliance with HIA best practices. A review of six EISs revealed that the CNSC is falling short of best practices and is ultimately failing to account for many nontrivial risks. The fact that the Pickering A Return to Service, Bruce A Restart/ Refurbishment and Pickering B Refurbishment EAs were all approved represents a "willful neglect of safety" by the proponents (Perrow, 2007:134), evidently permitted by the CNSC. The CNSC's "risk-informed" regulatory approach is clearly enabling approvals to be made without the full consideration of risk.

\subsection{Study Limitations}

The main limitation of this study lies with an important methodological assumption: the degree of concordance among authoritative sources in a literature review and interview process has been used to determine best practices. However, this is considered a reasonable assumption, as Zaring (2006) contended that best practices are more often a set of common practices. This statement, however, generates a new 
possible research limitation: was enough research conducted and were enough experts interviewed to claim the establishment of best practices? The general consensus in the interview process and the lack of identified gaps in the interview template lead the researcher to believe so.

Another possible constraint on the research findings was that unlike some published EA reviews (Lee et al., 1999; Gray and Edwards-Jones, 2003), the researcher was the sole EA reviewer. However, these published frameworks also relied on a lettergrading scale, in which discrepancies seem more likely than in a sector-specific framework requiring a binary response. Extensive efforts were taken to ensure each evaluation framework was filled out accurately and that the information needed to satisfy each criterion was held constant among the six EAs. The independent nature of this dissertation made this limitation unavoidable.

\subsection{Research Contribution and Future Application}

The work accomplished in this thesis contributes to the substantial research base of EA and HIA reviews. The research focus is unique, however, as the researcher is unaware of any other study solely focusing on EAs of nuclear power plants.

Furthermore, a comprehensive HIA best practice framework, based on the convergence of authoritative sources, was derived and may be used in similar future research endeavors. There are two possible ways in which the framework may be used: as evaluation criteria, similar to the present research, or as a scoping guide during the $E A$ process of nuclear power plant return to service/ refurbishment/ construction. One future opportunity to apply this checklist is to the EA for nuclear reactor construction at 
Peace River, Alberta, predicted to be released by BP in late 2010 (Chakravorty et al., 2010). It would be interesting to observe whether the various shortcomings identified in this research persist.

One final note is warranted. Ontario has recently legislated the Green Energy Act, which is encouraging the rapid development of renewable energy sources, thereby reducing the urgency of building large-scale nuclear power plants for centralized electricity production (Ministry of Energy and Infrastructure, 2010). The future of nuclear power is at a critical crossroads: many environmentalists have warmed up to the idea of nuclear power as a means to reduce greenhouse gas emissions (Poortinga et al., 2006), but cheaper and more efficient technologies are being developed, thus precluding the need for an expansion of nuclear power. It is the researcher's hope that if a nuclear power renaissance does indeed occur, the findings of this research will assist in improving the HIA process and ultimately help minimize undue risks to public health and safety.

\subsection{Summary}

An overall summary of the research methods was presented in this chapter. A discussion followed to assess the CNSC's overall compliance with best practices, which led into an examination of the most prominent shortcomings in individual EAs. Additionally, limitations of the study were identified and addressed. Finally, the contribution of this thesis to the literature was outlined and future research opportunities were identified. 


\section{REFERENCES}

Adler, M.A. 2004. Against 'individual risk': A sympathetic critique of risk assessment. University of Pennsylvania Law Review. 153:1121-1250.

Ali, S., O'Callaghan, V.O., Middleton, J.D. and Little, R. 2009. The challenges of evaluating a health impact assessment. Critical Public Health. 19(2):171-180.

Alvarez, R. 2002. What about spent fuel?. Bulletin of the Atomic Scientists. 58(1):45-47.

Ansolabehere, S., Deutch, J., Driscoll, M., Gray, P.E., Holdren, J.P., Joskow, P.L. et al. 2003. The Future of Nuclear Power. Massachusetts Institute of Technology.

Applegate, J.S. 1995. A beginning and not an end in itself: The role of risk assessment in environmental decision-making. University of Cincinnati Law Review. 63(4): 1643-1677.

Arai, A.B. 2001. Science and culture in the environmental state, The case of reactor layups at Ontario Hydro. Organization \& Environment. 14(4):409-424.

Arnstein, S. 1969. A ladder of citizen participation. Journal of the American Planning Association. 35:216-224.

Baker, P.J. and Hoel, D.G. 2007. Meta-analysis of standardized incidence and mortality rates of childhood leukemia in proximity to nuclear facilities. European Journal of Cancer Care. 16:355-363.

Baverstock, K. and Williams, D. 2006. The Chernobyl accident 20 years on: An assessment of the health consequences and the international response. Environmental Health Perspectives. 114(9):1312-1317.

Bekker, M.P.M., Putters, K. and van der Grinten, T.E.D. 2005. Evaluating the impact of HIA on urban reconstruction decision-making. Who manages whose risks?. Environmental Impact Assessment Review. 25:758-771.

Bertell, R. 2007. Comments on "Radiation \& Health in Durham Region" Report 2007. Prepared for IICPH, July.

Bhatia, R. 2007. Protecting health using an environmental impact assessment: A case study of San Francisco land use decisionmaking. American Journal of Public Health. 97(3):406-413.

Bhatia, R. and Wernham, A. 2008. Integrating human health into environmental impact assessment: An unrealized opportunity for environmental health and justice. Environmental Health Perspectives. 116(8):991-1000. 
Birley, M. 2003. Health impact assessment, integration and critical appraisal. Impact Assessment and Project Appraisal. 21(4):313-321.

Blake, E.M. 2005. The Canadian comeback. Nuclear News. 48(10):32-36.

Bodansky, D. 2004. Nuclear Energy- Principles, Practices, and Prospects. New York: Springer-Verlag.

Bredimas, A. and Nuttall, W.J. 2008. An international comparison of regulatory organizations and licensing procedures for new nuclear power plants. Energy Policy. 36:1344-1354.

Breeding, R.J., Helton, J.C., Gorham, E.D. and Harper, F.T. 1992. Summary description of the methods used in the probabilistic risk assessments for NUREG-1150. Nuclear Engineering and Design. 135:1-27.

Brennan, J. (Speaker). 2010. Nuclear Security Summit Press Briefing [Video]. Retrieved April 12, 2010 from http://www.youtube.com/watch?v=3WolR-q08L4.

Brenner, D.J., Doll, R., Goodhead, D.T., Hall, E.J., Land, C.E., Little, J.B. et al. 2003. Cancer risks attributable to low doses of ionizing radiation: assessing what we really know. Proceedings of the National Academy of Sciences of the United States of America. 100:13761-13766.

Briggs, D.J., Sabel, C.E. and Lee, K. 2009. Uncertainty in epidemiology and health risk and impact assessment. Environmental Geochemistry and Health. 31:189-203.

British Medical Association (BMA). 1999. Health and Environmental Impact Assessment: An Integrated Approach. London: Earthscan Publications Ltd.

Brooks, G.L. 1993. A Short History of the CANDU Nuclear Power System. Prepared for the Ontario Hydro Demand/Supply Plan Hearing, January.

Bruce Power (BP). 2002. Bruce A Restart Project Environmental Assessment Study Report. August.

Bruce Power (BP). 2005a. Bruce A Refurbishment for Life Extension and Continued Operations Project Environmental Assessment, Volume 1: Main Report. December.

Bruce Power (BP). 2005b. Bruce A Refurbishment for Life Extension and Continued Operations Project Environmental Assessment, Volume 2: Appendices. December. 
Bruce Power (BP). 2008a. Bruce New Nuclear Power Plant Project Environmental Assessment, Human Health and Safety Considerations Technical Support Document. September.

Bruce Power (BP). 2008b. Bruce New Nuclear Power Plant Project Environmental Assessment, Radioactivity Technical Support Document. May.

Bruce Power (BP). 2008c. Bruce New Nuclear Power Plant Project Environmental Assessment, Volume 1: Methods, Project Description, and Existing Environment. September.

Bruce Power (BP). 2008d. Bruce New Nuclear Power Plant Project Environmental Assessment, Volume 2: Assessment of Effects. September.

Bruce Power (BP). 2008e. Bruce New Nuclear Power Plant Project Environmental Assessment, Volume 3: Appendices. September.

Bruce Power gives prelim Alpha radiation reports to CNSC. 2010, February 18. The Kincardine News. Retrieved April 28, 2010 from http://kincardinenews.com/ArticleDisplay.aspx?archive=true\&e=2455620.

Buckley, J.D., Robison, L.L., Swotinsky, R., Garabrant, D.H., LeBeau, M., Manchester, P. et al. 1989. Occupational exposures of parents of children with acute nonlymphocytic leukemia: A report from the Childrens Cancer Study Group. Cancer Research. 49:4030-4037.

Buckthought, M. 2009. Tritium on Tap, Keep Radioactive Tritium Out of Our Drinking Water. Sierra Club of Canada, November.

Busby, C. and Fucic, A. 2006. Ionizing radiation and children's health. Acta Pediatrica. 95(453):81-85.

Busby, C., Lengfelder, E., Pflugbeil, S. and Schmitz-Feuerhake, I. 2009. The evidence of radiation effects in embryos and fetuses exposed to Chernobyl fallout and the question of dose response. Medicine, Conflict and Survival. 25(1):20-40.

Callahan, M.A. and Sexton, K. 2007. If cumulative risk assessment is the answer, what is the question?. Environmental Health Perspectives. 115(5):799-806.

Campbell-Mohn, C. and Applegate, J.S. 1999. Learning from NEPA: guidelines for responsible risk legislation. Harvard Environmental Law Review. 23:93-139.

Canadian Environmental Assessment Act (CEAA). 1992. Ottawa: House of Commons of Canada. 
Canadian Environmental Assessment Act (CEAA), Comprehensive Study List Regulations. 1994. Ottawa: House of Commons of Canada.

Canadian Nuclear Safety Commission (CNSC). 2004. Keeping Radiation Exposures and Doses "As Low as Reasonably Achievable (ALARA)", G-129. CNSC, October.

Canadian Nuclear Safety Commission (CNSC). 2005a. Canadian Nuclear Safety Commission 2004-2005 Annual Report. CNSC.

Canadian Nuclear Safety Commission (CNSC). 2005b. Probabilistic Safety Assessment (PSA) for Nuclear Power Plants. S-294. CNSC, April.

Canadian Nuclear Safety Commission (CNSC). 2007a. Annual CNSC Staff Report for 2006 on the Safety Performance of the Canadian Nuclear Power Industry, INFO-0761. CNSC, June.

Canadian Nuclear Safety Commission (CNSC). 2007b. Protecting Canadians, Canadian Nuclear Safety Commission 2006-2007 Annual Report. CNSC.

Canadian Nuclear Safety Commission (CNSC). 2008a. Annual CNSC Staff Report for 2007 on the Safety Performance of the Canadian Nuclear Power Industry. CNSC, August.

Canadian Nuclear Safety Commission (CNSC). 2008b. CNSC 2007-08 Annual Report. CNSC

Canadian Nuclear Safety Commission (CNSC). 2008c. Design of New Nuclear Power Plants, RD-337. CNSC, November.

Canadian Nuclear Safety Commission (CNSC). 2008d. Standards and Guidelines for Tritium in Drinking Water, INFO-0766. CNSC, January.

Canadian Nuclear Safety Commission (CNSC). 2009a. Completed. Retrieved April 28, 2010 from http://www.cnsc-ccsn.gc.ca/eng/ea/ealist/completed/index.cfm.

Canadian Nuclear Safety Commission (CNSC). 2009b. Completed, Proposed Bruce A refurbishment for life extension and continued operation. Retrieved April 26, 2010 from http://www.cnsc-ccsn.gc.ca/eng/ea/ealist/completed/

EA_04_01_8081.cfm.

Canadian Nuclear Safety Commission (CNSC). 2009c. Completed, Proposed Pickering $B$ refurbishment and continued operation. Retrieved April 26, 2010 from http://www.cnsc-ccsn.gc.ca/eng/ea/ealist/completed/EA_06_01_21226.cfm. 
Canadian Nuclear Safety Commission (CNSC). 2009d. Joint Review Panel for the Bruce Power New Nuclear Power Plant Project. Retrieved April 6, 2010 from http://www.cnsc-ccsn.gc.ca/eng/commission/members/jrp/index.cfm.

Canadian Nuclear Safety Commission (CNSC). 2009e. Joint Review Panel for Proposed Darlington New Nuclear Power Plant Project. Retrieved April 6, 2010 from http://nuclearsafety.gc.ca/eng/commission/members/jrp/darlington/ index.cfm.

Canadian Nuclear Safety Commission (CNSC). 2009f. List of EAs. Retrieved April 29, 2010 from http://www.cnsc-ccsn.gc.ca/eng/ea/ealist/index.cfm.

Canadian Nuclear Safety Commission (CNSC). 2010. Status of New Nuclear Projects. Retrieved April 22, 2010 from http://www.nuclearsafety.gc.ca/eng/readingroom/newbuilds/index.cfm.

Canter, L. 1993. Pragmatic suggestions for incorporating risk assessment principles in EIA studies. The Environmental Professional. 15:125-138.

Cardis, E., Vrijheid, M., Blettner, M., Gilbert, E., Hakama, M. and Hill, C. 2007. The 15country collaborative study of cancer risk among radiation workers in the nuclear industry: Estimates of radiation-related cancer risks. Radiation Research. 167(4):396-416.

Chakravorty, U., Haluza-Delay, R., Lin, J. and Parkins, J. 2010. Nuclear Energy in Alberta: What You Need to Know. D.J. Davidson (Ed.) Environmental Research and Studies Centre, January.

Committee Examining Radiation Risks from Internal Emitters (CERRIE). 2004. Reports of the Committee Examining Radiation Risks from Internal Emitters. Chilton: National Radiological Protection Board.

Cole, B.L. and Fielding, J.E. 2007. Health impact assessment: A tool to help policy makers understand health beyond health care. Annual Review of Public Health. 28: 393-412.

Cole, B.L, Shimkhada, R., Fielding, J.E., Kominski, G. and Morgenstern, H. 2005. Methodologies for realizing the potential of health impact assessment. American Journal of Preventative Medicine. 28(4):382-389.

Copplestone, D., Sutherland, A., McGoff, A., Bennett, D., Matadeen, A., Mok, F. et al. 2009. Study of historic nuclear reactor discharge data. Radioprotection. 44(5): 875-880. 
Cwikel, J.G., Gidron, Y. and Quastel, M. 2010. Low-dose environmental radiation, DNA damage, and cancer: The possible contribution of psychological factors. Psychology, Health \& Medicine. 15(1):1-16.

da Cruz, A.D., de Melo e Silva, D., da Silva, C.C., Nelson, R.J., Ribeiro, L.M., Pedrosa, E.R. et al. 2008. Microsatellite mutations in the offspring of irradiated parents 19 years after the Cesium-137 accident. Mutation Research. 652:175-179.

Davies, K. and Sadler, B. 1997. Environmental Assessment and Human Health: Perspectives, Approaches, and Future Directions. Ottawa: Health Canada.

Demidova, O., and Cherp, A. 2005. Risk assessment for improved treatment of health considerations in EIA. Environmental Impact Assessment Review. 25(4): 411-429.

Diduck, A. and Sinclair, A.J. 2002. Public involvement in environmental assessment: The case of the nonparticipant. Environmental Management. 29(4):578-588.

Dodic-Fikfak, M., Clapp, R. and Kriebel, D. 1999. The health risks of decommissioning nuclear facilities. New Solutions. 9(2):153-161.

Doelle, M. and Sinclair, A.J. 2006. Time for a new approach to public participation in EA: Promoting cooperation and consensus for sustainability. Environmental Impact Assessment Review. 26:185-205.

Doull, J. 2003. The "Red Book" and other risk assessment milestones. Human \& Ecological Risk Assessment. 9(5):1229-1238.

Dubrova, Y.E. 2003. Long-term genetic effects of radiation exposure. Mutation Research. 544:433-439.

Dubrova, Y.E., Bersimbaev, R.I., Djansugurova, L.B., Tankimanova, M.K., Mamyrbaeva, Z.Z., Mustonen, R. et al. 2002. Nuclear weapon tests and human germline mutation rate. Science. 295:1037.

Easton, K.L., McComish, J.F. and Greenberg, R. 2000. Avoiding common pitfalls in qualitative data collection and transcription. Qualitative Health Research. 10 (5):703-707.

Evans, R.D. 1933. Radium poisoning, A review of present knowledge. American Journal of Public Health and The Nations Health. 23(10):1017-1023.

Eyles, J.D. 1999 Health, environmental assessments and population heath: Tools for a complex process. Canadian Journal of Public Health. 90(1):S31-34. 
Fairlie, I. 2005. Uncertainties in doses and risks from internal radiation. Medicine, Conflict and Survival. 21(2):111-126.

Fairlie, I. 2007. Tritium hazard report: pollution and radiation risk from Canadian nuclear facilities. Toronto: Greenpeace Canada, June.

Fairlie, I. 2008. The hazards of tritium - revisited. Medicine, Conflict and Survival. 24 (4):306-319.

Fairlie, I. 2010. Childhood cancer near German nuclear power stations. Journal of Environmental Science and Health Part C. 28:1-21.

Fairlie, I. and Sumner, D. 2006. The Other Report on Chernobyl (TORCH), An independent scientific evaluation of health and environmental effects 20 years after the nuclear disaster providing critical analyses of a recent report by the International Atomic Energy Agency (IAEA) and the World Health Organization (WHO). Commissioned by Rebecca Harris, MEP/EFA in the European Parliament.

Finan, A.E., Miu, K. and Kadak, A.C. 2006. Nuclear technology and Canadian oil sands: Integration of nuclear power with in-situ oil extraction. Proceedings, 2006 International Congress on Advances in Nuclear Power Plants, ICAPP06.

Finley, B., Proctor, D., Scott, P., Harrington, N., Paustenbach, D. and Price, P. 1994. Recommended distribution for exposure factors frequently used in health risk assessment. Risk Analysis. 14(4):533-553.

Forester, J. 1982. Planning in the face of power. Journal of the American Planning Association. 48(1):67-80.

Fullwood, R.R. 2000. Probabilistic Safety Assessment in the Chemical and Nuclear Industries. Boston: Buttersworth-Heinemann.

Galeriu, D., Paunescu, N., Mocanu, N. and Melintescu, A. 2003. A data base for tritium level around a chronic source, Report WP3 IDRANAP 58-03/2003. European Commission Center of Excellence, IDRANAP.

Garrick, B.J. and Christie, R.F. 2002. Probabilistic risk assessment practices in the USA for nuclear power plants. Safety Science. 40:177-201.

Goodhead, D.T. 2009. The relevance of dose for low-energy beta emitters. Journal of Radiological Protection. 29:321-333.

Gray, I. and Edwards-Jones, G. 2003. Quality review of ESs, A review of environmental statements in the British forest sector. Impact Assessment and Project Appraisal. 21(4):303-312. 
Gronlund, L., Lochbaum, D. and Lyman, E. 2007. Nuclear Power in a Warming World, Assessing the Risks, Addressing the Challenges. Cambridge: UCS Publications, December.

Guest, G., Bunce, A. and Johnson, L. 2006. How many interviews are enough? An experiment with data saturation and variability. Field Methods. 18(1):59-82.

Hamby, D.M. 1999. Uncertainty of the tritium dose conversion factor. Health Physics. 77 (3):291-297.

Hanna, K.S. 2005.A Brief Introduction to Environmental Impact Assessment. In Hanna, K. S. (Ed.) Environmental Impact Assessment: Practice and Participation in Canada. (pp. 3-15). Toronto: Oxford University Press.

Hanna, K.S. and Gibson, R.B. 2005. Progress and Uncertainty: The Evolution of Federal Environmental Assessment in Canada. In Hanna, K. S. (Ed.) Environmental Impact Assessment: Practice and Participation in Canada. (pp. 16-32). Toronto: Oxford University Press.

Hansell, A. and Aylin, P. 2003. Use of health data in health impact assessment. Impact Assessment and Project Appraisal. 21(1):57-64.

Harris, P.J., Harris, E., Thompson, S., Harris-Roxas and Kemp, L. 2009. Human health and wellbeing in environmental impact assessment in New South Whales, Australia: Auditing health impacts within environmental assessments of major projects. Environmental Impact Assessment Review. doi: 10.1016/j.eiar. 2009.02.002.

Hartsough, D.M. and Savitsky, J.C. 1984. Three Mile Island:Psychology and environmental policy at a crossroads. American Psychologist. 39(10):1113-1122.

Harvey, P.D. 1990. Educated guesses: Health risk assessment in environmental impact statements. American Journal of Law and Medicine. 16(3):399-427.

Hayns, M.R. 1999. The evolution of probabilistic risk assessment in the nuclear industry. Trans I ChemE. 77(Part B):117-142.

Hazell, S. 1999. Canada v. The Environment: Federal Environmental Assessment 1984-1998. Toronto: Canadian Environmental Defence Fund.

Health Canada. 2004a. Canadian Handbook on Health Impact Assessment Volume 1:The basics. Ottawa: Government of Canada.

Health Canada. 2004b. Canadian Handbook on Health Impact Assessment Volume 2:Approaches and Decision-making. Ottawa: Government of Canada. 
Health Canada. 2004c. Canadian Handbook on Health Impact Assessment Volume 3:The Multidisciplinary Team. Ottawa: Government of Canada.

Health Canada. 2004d. Canadian Handbook on Health Impact Assessment Volume 4:Health impacts by industry sector. Ottawa: Government of Canada.

Health Protection Agency (HPA). 2007. Review of Risks from Tritium, Report of the Independent Advisory Group on lonising Radiation. London: HPA, November.

Herring, R.J. 2005. The Canadian Federal EIA System. In Hanna, K. S. (Ed.) Environmental Impact Assessment: Practice and Participation in Canada. (pp. 231-247). Toronto: Oxford University Press.

Hill, B. 1965. The environment and disease: Association or causation?. Proceedings of the Royal Society of Medicine. 58:295-300.

Huang, Y. and Batterman, S. 2000. Residence location as a measure of environmental exposure: a review of air pollution epidemiology studies. Journal of Exposure Analysis and Environmental Epidemiology. 10:6-85.

Huff, J.H. 2007. Industry influence on occupational and environmental public health. International Journal of Occupational and Environmental Health. 13:107-117.

International Committee on Radiological Protection (ICRP) 101. 2006. Assessing dose of the representative person for the purpose of radiation protection of the public and the optimization of radiological protection: Broadening the process. Annals of the ICRP. Volume 36, 3.

Janisch, H.N. 1996. In Search of the Cat's Pyjamas: Regulatory Institutions and the Restructured Industry. In Daniels, R.J. (Ed.) Ontario Hydro at the Millennium, Has Monopoly s Moment Passed?. (pp. 355-390). University of Toronto Faculty of Law.

Jardine, C.G., Hrudey, S.E., Shortreed, J.H., Craig, L., Krewski, D., Furgal, C. et al. 2003. Risk management frameworks for human health and environmental risks. Journal of Toxicology and Environmental Health. 6:569-641.

Kaatsch, P., Spix, C., Schulze-Rath, R., Schmiedel, S. and Blettner, M. 2008. Leukaemia in young children living in the vicinity of German nuclear power plants. International Journal of Cancer. 1220:721-726.

Kearney, M. 2004. Walking the walk? Community participation in HIA. Environmental Impact Assessment Review. 24(2): 217-229. 
Keller, W. and Modarres, M. 2005. A historical overview of probabilistic risk assessment development and its use in the nuclear power industry: a tribute to the late Professor Norman Carl Rasmussen. Reliability Engineering \& System Safety. 89:271-285.

Kemm, J., Parry, J. and Palmer, S. (Eds.) 2004. Health Impact Assessment: Concepts, Theory, Techniques, and Applications. Oxford: Oxford University Press.

Kirchsteiger, C. 2006. Current practices for risk zoning around nuclear power plants in comparison to other industry sectors. Journal of Hazardous Materials. 136:392-397.

Krewitt, W. 2002. External costs of energy- do the answers match the questions? Looking back at 10 years of ExternE. Energy Policy. 30:839-848.

Kwiatkowski, R.E. 1998. The role of risk assessment and risk management in environmental assessment. Environmetrics. 9:587-598.

Kwiatkowski, R.E. and Ooi, M. 2003. Integrated environmental impact assessment: a Canadian example. Bulletin of the World Health Organization. 81(6):434-438.

Lee, N., Colley, R., Bonde, J. and Simpson, J. 1999. Reviewing the quality of environmental statements and environmental appraisals, Occasional Paper Number 55. Manchester: Department of Planning and Landscape, University of Manchester.

Lemstra, M. 2009. Exposure to Radiation and Health Outcomes. Regina: Canadian Centre for Policy Alternatives, April.

Little, M.P and Wakeford, R. 2008. Systematic review of epidemiological studies of exposure to tritium. Journal of Radiological Protection. 28:9-32.

Lochbaum, D. 2000. Nuclear Plant Risk Studies, Failing the Grade. Cambridge: UCS Publications, August.

Lochbaum, D. 2004. U.S. Nuclear Plants in the 21st Century, The Risk of a Lifetime. Cambridge: UCS Publications, May.

Lowrance, W. 1975. Of Acceptable Risk: Science and the Determination of Safety. New York: WA Benjamin.

Makhijani, A. 2008. The Use of the Reference Man in Radiation Protection Standards and Guidance with Recommendations for Change. Report prepared for the Institute for Energy and Environmental Research, December. 
Makhijani, A., Smith, B. and Thorne, M.C. 2006. Science for the Vulnerable: Setting Radiation and Multiple Exposure Environmental Health Standards to Protect Those Most at Risk. Report prepared for the Institute for Energy and Environmental Research, October.

Mangano, J.J. 2006. A short latency between radiation exposure from nuclear plants and cancer in young children. International Journal of Health Services. 36(1): 113-135.

Mangano, J.J. 2009. Geographic variation in U.S. thyroid cancer incidence and a cluster near nuclear reactors in New Jersey, New York, and Pennsylvania. International Journal of Health Services. 39(4):643-661.

McLaughlin, T.P., Monahan, S.P., Pruvost, N.L., Frolov, V.V., Ryazanov, B.G. and Sviridov, V.I. 2000. A review of criticality accidents, 2000 revision, LA-13638. Los Alamos: Los Alamos National Laboratory.

McMullan, C. and Eyles, J. 1999. Risky business: An analysis of claimsmaking in the development of an Ontario drinking water objective for tritium. Social Problems. 46(2):294-311.

Meho, L.I. 2006. E-mail interviewing in qualitative research: A methodological discussion. Journal of the American Society for Informational Science and Technology. 57(10):1284-1295.

Miller, G. 2008. Getting to K(no)w: Annual Report, 2007-2008. Toronto: Environmental Commissioner of Ontario.

Mindell, J.S., Boltong, A. and Forde, I. 2008. A review of health impact assessment frameworks. Public Health. 122:1177-1187.

Ministry of Energy and Infrastructure. 2010. Ontario s Green Energy Act, Our Path to a Green Economy and a Cleaner Environment. Retrieved on April 29, 2010 from http://www.mei.gov.on.ca/en/energy/gea/.

Mittica, P. 2007. Chernobyl: The Hidden Legacy. With additional text by N. Rosenblum, R. Bertell, and W. Tcherkoff. London: Trolley Ltd.

Mosey, D. 2006. Reactor Accidents: Institutional Failure in the Nuclear Industry, 2nd Edition. Guildford: Nuclear Engineering International Special Publications.

Mueller, C.J. 1996. Facilitating Relative Comparisons of Health Impacts from Postulated Accidents in Environmental Impact Statements. Argonne: Argonne National Laboratory. 
Muirhead, C.R. 2008. Exposure assessment: Implications for epidemiological studies of ionizing radiation. Radiation Protection Dosimetry. 132(2):134-138.

Mulvihill, P.R. 2003. Expanding the scoping community. Environmental Impact Assessment Review. 23:39-49.

National Academy of Sciences (NAS). 2006. Health Risks from Exposure to Low Levels of lonizing Radiation: BEIR VII Phase 2. Washington: National Academy Press.

Nieuwenhuijsen, M., Paustenbach, D. and Duarte-Davidson, R. 2006. New developments in exposure assessment: The impact on the practice of health risk assessment and epidemiological studies. Environment International. 32:996-1009.

Noble, B. F. 2006. Introduction to Environmental Impact Assessment: A Guide Principles and Practice. Toronto: Oxford University Press.

Noble, B. and Storey, K. 2005. Towards increasing the utility of follow-up in Canadian EIA. Environmental Impact Assessment Review. 25:163-180.

Noshchenko, A.G., Zamostiyan, P.V., Bondar, O.Y. and Drozdova, D. 2002. Radiationinduced leukemia risk among those aged 0-20 at the time of the Chernobyl accident: A case-control study in the Ukraine. International Journal of Cancer. 99:609-618.

Nuclear Energy Agency (NEA), Committee on the Safety of Nuclear Installations. 2009. Probabilistic Safety Analysis (PSA) of Other External Events than Earthquake, $N E A / C S N I / R(2009) 4$. March.

Nuclear Safety and Control Act (NSCA). 1997. Ottawa: House of Commons of Canada.

Nussbaum, R.H. 1998. The linear no-threshold dose-effect relation: Is it relevant to radiation protection regulation?. Medical Physics. 25(3): 291-299.

Nussbaum, R. 2007. The Chernobyl nuclear catastrophe: Unacknowledged health detriment. Environmental Health Perspectives. 115(5):238-239.

Nussbaum, R. 2009. Childhood leukemia and cancers near German nuclear reactors: Significance, context, and ramifications of recent studies. International Journal of Occupational Health. 15:318-323.

O'Connor, M. and van den Hove, S. 2001. Prospects for public participation on nuclear risks and policy options: Innovations in governance practices for sustainable development in the European Union. Journal of Hazardous Materials. 86:77-99. 
Ontario Power Authority (OPA). 2008. The Integrated Power System Plan for the Period 2008-2027, EB-2007-0707. August.

Ontario Power Generation (OPG). 2000a. Building on Our Foundation, Ontario Power Generation Inc. 1999 Annual Report. OPG.

Ontario Power Generation (OPG). 2000b. Pickering A Return to Service Environmental Assessment. April.

Ontario Power Generation (OPG). 2000c. Pickering A Return to Service Environmental Assessment, Technical Support Document Radiation and Radioactivity. April.

Ontario Power Generation (OPG). 2007a. Credible Malfunctions and Accident Scenarios Technical Support Document, Refurbishment and Continued Operation of Pickering B Nuclear Generating Station Environmental Assessment, NK30-REP-07701-00014. December.

Ontario Power Generation (OPG). 2007b. Human Health Technical Support Document, Refurbishment and Continued Operation of Pickering B Nuclear Generating Station Environmental Assessment, NK30-REP-07701-00015. December.

Ontario Power Generation (OPG). 2007c. Radiation and Radioactivity Technical Support Document, Refurbishment and Continued Operation of Pickering B Nuclear Generating Station Environmental Assessment, NK30-REP-07701-00004. December.

Ontario Power Generation (OPG). 2007d. Refurbishment and Continued Operation of Pickering B Nuclear Generating Station Environmental Assessment,NK30REP-07701-00002. December.

Ontario Power Generation (OPG). 2008. Pickering B Risk Assessment Summary Report, NK30-REP-03611-00005. November.

Ontario Power Generation (OPG). 2009a. Emergency Planning and Preparedness Technical Support Document, New Nuclear - Darlington Environmental Assessment, NK054-REP-07730-00021. August.

Ontario Power Generation (OPG). 2009b. Human Health Technical Support Document, New Nuclear - Darlington Environmental Assessment, NK054REP-07730-00025. September.

Ontario Power Generation (OPG). 2009c. Malfunctions, Accidents and Malevolent Acts Technical Support Document, New Nuclear - Darlington Environmental Assessment, NK054-REP-07730-00024. September. 
Ontario Power Generation (OPG). 2009d. New Nuclear - Darlington Environmental Assessment. September.

Ontario Power Generation (OPG). 2009e. Radiation \& Radioactivity Environment Assessment of Environmental Effects Technical Support Document, New Nuclear - Darlington Environmental Assessment, NK054-REP-07730-00018. August.

Ontario Power Generation (OPG). 2009f. Radiation \& Radioactivity Environment Existing Environmental Conditions Technical Support Document, New Nuclear Darlington Environmental Assessment, NK054-REP-07730-00008. September.

Oxman, A.D., Shannon, H.S., Garland, W.J. and Torrance, G.W. 1989. Nuclear safety in Ontario: A critical review of quantitative analyses. Risk Analysis. 9(1):43-54.

Ozonoff, D. 1994. Conceptions and misconceptions about human health impact analysis. Environmental Impact Assessment Review. 14:311-319.

Parry, J.M. and Kemm, J.R. 2005. Criteria for use in the evaluation of health impact assessments. Public Health. 119:1122-1129.

Paustenbach, D.J. 2000. The practice of exposure assessment: A state-of-the-art review. Journal of Toxicology and Environmental Health, Part B. 3:179-291.

Perrow, C. 1999. Normal Accidents: Living with High Risk Technologies. New York: Basic Books.

Perrow, C. 2007. The Next Catastrophe, Reducing Our Vulnerabilities to Natural, Industrial, and Terrorist Disasters. Princeton: Princeton University Press.

Petticrew, M., Cummins, S., Sparks, L. and Findlay, A. 2007. Validating health impact assessment: prediction is difficult (especially about the future). Environmental Impact Asssessment Review. 27:101-107.

Poortinga, W., Pidgeon, N.F. and Lorenzoni, I. 2006. Public perceptions of nuclear power, climate change and energy options in Britain: Summary findings of a survey conducted during October and November 2005. Technical Report (Understanding risk working paper 06-02). Norwich: Centre for Environmental Risk.

Quigley, R.C. and Taylor, L.C. 2004. Evaluating health impact assessment. Public Health. 118:544-552

Quigley, R., den Broeder, L., Furu, P., Bond, A., Cave, B. and Bos, R. 2006. Health Impact Assessment International Best Practice Principles. Special Publication Series No. 5. Fargo:International Association for Impact Assessment. 
Rahn, R.O. and Upton, A.C. 2007. Radiological risk assessment. In Robson, M.G. and Toscano, W.A. (Eds.) Risk Assessment for Environmental Health. (pp. 239-283). San Francisco: Jossey-Bass.

Rahu, M. 2003. Health effects of the Chernobyl accident: fears, rumors and the truth. European Journal of Cancer. 39:295-299.

Rattle, R. and Kwiatkowski, R.E. 2003. Integrating health and social impact assessment. In H.A. Becker \& F. Vanclay (Eds.) The international handbook on social impact assessment: Conceptual and methodological advances. (pp. 92-107). Cheltenham: Edward Elgar.

Richardson, D., Wing, S. and Stewart, A. 1997. Epidemiologic studies of the effects of exposure to ionizing radiation. Schleswig-Holstein: Ministry of Finance and Energy.

Richardson, R.B. 2009. Factors that elevate the internal radionuclide and chemical retention, dose and health risks to infants and children in a radiological-nuclear emergency. Radiation Protection Dosimetry. 134(3-4):167-180.

Risk of restarting nuclear reactor too high: Keen. 2008, January 29. CBC News. Retrieved May 24, 2009 from http://www.cbc.ca/canada/story/2008/01/29/ isotopes-keen.html.

Ritz, B., Morgenstern, H., Crawford-Brown, D. and Young, B. 2000. The effects of internal radiation exposure on cancer mortality in nuclear workers at Rocketdyne/ Atomics International. Environmental Health Perspectives. 108(8): 743-751.

Rogers, G.O. 1998. Siting potentially hazardous facilities: what factors impact perceived and acceptable risk?. Landscape and Urban Planning. 39:265-281.

Rooney, C., Beral, V., Maconochie, N., Fraser, P. and Davies, G. 1993. Case-control study of prostatic cancer in employees of the United Kingdom Atomic Energy Authority. British Medical Journal. 307(27):1391-1397.

Salbu, B, Lind, O.C. and Skipperud, L. 2004. Radionuclide speciation and its relevance in environmental impact assessments. Journal of Environmental Radioactivity. 74:233-242.

Scherer, K., Kraut, A., Yassi, A., Wajda, A. and Bebchuk, J. 1994. Using administrative health data to monitor potential adverse health effects in environmental studies. Environmental Research. 66:143-151.

Seidman, I.E. 1991. Interviewing as Qualitative Research. New York: Teachers College Press. 
Shimizu, Y., Kodama, K., Nishi, N., Kasagi, F., Suyama, A., Soda, M. et al. 2010. Radiation exposure and circulatory disease risk: Hiroshima and Nagasaki atomic bomb survivor data, 1950-2003. British Medical Journal. 340:b5349.

Shrader-Frechette, K. 1993. Burying Uncertainty: Risk and the Case Against Geological Disposal of Nuclear Waste. Berkeley: University of California Press.

Shlyakhter, A. and Wilson, R. 1992. Chernobyl: the inevitable results of secrecy. Public Understanding of Science. 1:251-259.

Sobh, R. and Perry, C. 2006. Research design and data analysis in realism research. European Journal of Marketing. 40(11/12):1194-1209.

Sont, W.N., Zielinski, J.M., Asmore, J.P., Jiang, H., Krewski, D., Fair, M.E. et al. 2001. First Analysis of Cancer incidence and occupational radiation exposure based on the National Dose Registry of Canada. American Journal of Epidemiology. 153 (4):309-318.

Spiegel, J. and Yassi, A. 1997. The use of health indicators in environmental assessment. Journal of Medical Systems. 21(5):275-289.

Steinhäusler, F. 2009. Countering security risks to nuclear power plants. International Journal of Nuclear Governance, Economy and Ecology. 2(4):375-386.

Steinemann, A. 2000. Rethinking human health impact assessment. Environmental Impact Assessment Review. 20:627-645.

Steinemann, A. 2001. Improving alternatives for environmental impact assessment. Environmental Impact Assessment Review. 21:3-21.

Sturges, J.E. and Hanrahan, K.J. 2004. Comparing telephone and face-to-face qualitative interviewing: a research note. Qualitative Research. 4(1):107-118.

Sues, R.H., Amico, P.J. and Campbell, R.D. 1990. Significance of earthquake risk in nuclear power plant probabilistic risk assessments. Nuclear Engineering and Design. 123:27-44.

The Standing Senate Committee on Energy, The Environment and Natural Resources. 2001. Canada s Nuclear Reactors: How Much Safety is Enough?. Interim Report.

Thompson, G. 2000. A Review of the Accident Risk Posed by the Pickering A Nuclear Generating Station, A Report to the Standing Committee on Energy, Environment and Natural Resources of the Canadian Senate. Cambridge: Institute for Resource and Security Studies, August. 
Thompson, G. 2003. Robust Storage of Spent Nuclear Fuel: A Neglected Issue of Homeland Security. Cambridge: Institute for Resource and Security Studies, January.

Thompson, G.R. 2008a. Risks of Operating CANDU 6 Nuclear Power Plants: Gentilly Unit 2 Refurbishment and its Global Implications. Cambridge: Institute for Resource and Security Studies, October.

Thompson, G.R. 2008b. Scope of the Environmental Impact Statement for New Nuclear Power Plants at the Bruce Site in Ontario: Assessment of Accidents and Malfunctions. Cambridge: Institute for Resource and Security Studies, June.

Thompson, G.R. 2008c. Scope of the Environmental Impact Statement for New Nuclear Power Plants at the Darlington Site in Ontario: Accidents, Malfunctions and the Precautionary Approach. Cambridge: Institute for Resource and Security Studies, November.

Tickner, J.A. 2007. Why risk assessment is not enough to protect health. In Robson, M.G. and Toscano, W.A. (Eds.) Risk Assessment for Environmental Health. (pp. 423-460). San Francisco: Jossey-Bass.

Till, J.E. 2008. The Radiological Assessment Process. In Till, J.E. and Grogan, H.A. (Eds.) Radiological Risk Assessment and Environmental Analysis. (pp. 1-28). New York: Oxford University Press.

United Nations Scientific Committee on the Effects of Atomic Radiation (UNSCEAR). 2000. Sources and Effects of lonizing Radiation, Volume II: Effects, Annex H: Combined Effects of Radiation and Other Agents. New York: United Nations.

United Nations Scientific Committee on the Effects of Atomic Radiation (UNSCEAR). 2006. Effects of lonizing Radiation, UNSCEAR 2006 Report to the General Assembly with Scientific Annexes, Vols I and II. New York: United Nations.

United States Environmental Protection Agency (USEPA). 2003. Framework for Cumulative Risk Assessment. EPA/600/P-02/001F. Washington: USEPA, Office of Research and Development.

United States Environmental Protection Agency (USEPA). 2008. EPA Radiogenic Cancer Risk Models and Projections for the U.S. Population, Draft. Washington: USEPA, December.

United States Nuclear Regulatory Commission (USNRC). 1990. Severe Accident Risks: An Assessment for Five U.S. Nuclear Power Plants, Final Summary Report, NUREG-1150, Volume 1. Washington: USNRC. 
United States Nuclear Regulatory Commission (USNRC). 2007. Probabilistic Risk Assessment (PRA). Retrieved May 25, 2010 from http://www.nrc.gov/images/ about-nrc/regulatory/risk-informed/pra6-r1.gif.

Veerman, J.L., Mackenbach, J.P. and Barendregt, J.J. 2007. Validity of predicitons in health impact assessment. Journal of Epidemiology and Community Health. 61:362-366.

Voillequé, P.G. 2008. Radionuclide Source Terms. In Till, J.E. and Grogan, H.A. (Eds.) Radiological Risk Assessment and Environmental Analysis. (pp. 31-69). New York: Oxford University Press.

von Stackelberg, K. \& Burmaster, D.E. 1994. A discussion on the use of probabilistic risk assessment in human health impact assessment. Environmental Impact Assessment Review. 14:385-401.

Walker, J.S. 2000. Permissible Dose: A History of Radiation Protection in the Twentieth Century. Berkeley: University of California Press.

Wall, I.B. 1980. Probabilistic Risk Assessment in nuclear power plant regulation. Nuclear Engineering and Design. 60:11-24.

Wernham, A. 2007. Inupiat health and proposed Alaskan oil development: Results of the first integrated health impact assessment/environmental impact statement for proposed oil development on Alaska's north slope. EcoHealth. 4:500-513.

Winfield, M., Jamison, A., Wong, R. and Czajkowski, P. 2006. Nuclear Power in Canada: An Examination of Risks, Impacts and Sustainability. The Pembina Institute.

Wing, S. 2003. Objectivity and ethics in environmental health science. Environmental Health Perspectives. 111(14):1809-1818.

Wing, S., Richardson, D., Armstrong, D. and Crawford-Brown, D. 1997. A reevaluation of cancer incidence near the Three Mile Island nuclear plant: The collision of evidence and assumptions. Environmental Health Perspectives. 105(1):52-57.

Wood, G. 2008. Thresholds and criteria for evaluating and communicating impact significance in environmental impact statements: 'See no evil, hear no evil, speak no evil'?. Environmental Impact Assessment Review. 28:22-38.

World Health Organization (WHO). 1994. Nuclear Power and Health: The Implications for Health of Nuclear Power Production. WHO Regional Publications. European Series No. 51.

Yap, N. T. 2003. Towards an Inclusive Framework for EA. Research and Development Monograph Series. 
Zandvoort, H. 2008. Risk zoning and risk decision making. International Journal of Risk Assessment and Management. 8(1/2):3-18.

Zaring, D. 2006. Best Practices. New York University Law Review. 294:331-334.

Zou, B., Wilson, J.G., Zhan, F.B. and Zeng, Y. 2009. Air pollution exposure assessment methods utilized in epidemiological studies. Journal of Environmental Monitoring $11: 475-490$. 


\section{Appendix A: Interview Template}

\section{Interview Questions}

\section{All of the following questions relate to Health Risk Assessments (HRAs) of nuclear power plants.}

1. Discuss the necessary parameters to identify a) the exposed population, b) sensitive/special populations

2. Identify any baseline information that would be necessary to undertake effective monitoring of the exposed population's health.

3. Discuss any priority safety issues of new plants that ought to require special attention.

4. Identify any specific parts that ought to be replaced during refurbishment and any monitoring changes that may be necessary.

5. Identify and discuss the most prominent authority from which to base HRA standards.

6. Discuss the use of precedents from previous HRAs.

7. There are several different models of low level radiation, e.g. linear no-threshold, hormesis; discuss applications of these in HRA.

8. Discuss the ideal/maximum number of individuals that ought to be exposed to routine emissions.

9. Discuss any information the public ought to be entitled to know regarding spills/ elevated emissions.

10. Discuss the use of perceived risk in HRA.

11. Define the public's role in HRA.

12. Discuss the use of chronic non-cancer effects in HRA.

13. Discuss the use of hydrazine in HRA.

14. Identify what drinking water standards for tritium ought to be. 
15. Discuss how uncertainty ought to be defined in HRA.

16. There are three levels of Probabilistic Risk Assessment (PRA):

Level 1: Determines the risk of various causes of core damage.

Level 2: Determines the risk of core damage leading to a large scale release.

Level 3: Determines the offsite consequences of a large scale release.

Discuss the role of PRA in HRA.

17. Discuss the need to independently review HRA.

18. Please identify any remaining topics that ought to be included in HRA Best Practices that have not yet been discussed. 


\section{Appendix B: Best Practice Framework}

\section{Health Risk Assessment Evaluation Package for Nuclear Power Plant Projects}

Year:

Title:

Type of EA:

Location:

Proponent:

EIS Prepared by:

Responsible Authority:

Each question requires a 'Yes' or 'No response to state whether each indicator is present in the document.

\section{Hazard Identification}

1.1. Description of each proposed reactor technology/part to be refurbished? 1234

1.1.1. Refurbishment/New Reactor- specific mention of the safety features of each proposed design? 156789101921107

1.1.1.1. Outlines differences in quantity/type of hazardous emissions for each alternative? 11119105

1.2. Identifies all possible health hazards distinctly for each phase of project: construction/refurbishment, operation, decommissioning? 1121314107113114

1.3. Considers increasing reactor emissions (e.g. tritium) during operational life (accommodates differences via probabilistic distribution of emission values)? 510 1516175999108120

1.4. Discussion of accident scenarios by use of Probabilistic Risk Assessment (PRA) for each proposed technology? 2791819202122101106117118119121

1.4.1. Includes both internal (e.g., equipment failure) and external (e.g., tornadoes) events? 9232425106117118119121128

1.4.1.1. Includes security/terrorism threats in external events? 92627117

1.4.2. Defines upper limit source terms for releases to the environment for all significant accident sequences? 2021222329303132101106117118119123

1.5. Discussion of wastes? 1419101107

1.5.1. Mentions types and quantities (including decommissioning, e.g., asbestos)? ${ }^{14}$ 19

1.5.3. Discusses method of long-term disposal? 1419101

\section{Evaluation}

1.1.

1.1.1.

1.1.1.1.

1.2.

1.3.

1.4 .
1.4.1.

1.4.1.1.

1.4.2.

1.5 .

1.5.1.

1.5.2. 
Summarize any strengths and weaknesses and state whether this stage was completed sufficiently overall.

\section{Exposure Assessment}

2.1. Numerous parameters used to identify exposed population? 81011161822333435 120122123

2.1.1. Description of geographic area of facility site? 119107

2.1.2. All possible exposure pathways described and justified? 11163436373839101111 114120

2.1.3. Consulted public about behavior habits to identify any unique groups? 481122 4041

2.1.4. Higher exposure/vulnerable groups identified in population (e.g., unborn fetus)? 717224243101105111

2.1.5. Describes species of radionuclides during environmental transport? 111631105 123

2.2. Describes baseline (prior to reactor construction/expansion) health information of exposed population? 671019363740444546101111114122

2.3. Evidence of completed environmental monitoring to detect baseline levels of all relevant radionuclides in areas with existing facilities? 811142239495051101122

2.3.1. Use of this data in HRA calculations? 111622

2.3.2. Use of data from past facility monitoring/EAs/HRAs to verify/validate models? 81649505294101112

2.3.3. Range (probabilistic distribution) of radiation exposure values used for public/worker exposure (Monte Carlo Analysis)? 161719343999113120

2.3.4. Upper and lower dose ranges given for workers (e.g., includes higher exposure activities? 16445354102113120

2.3.4.1. Internal (alpha, beta) and external (gamma) dosimetry for workers practiced? 343553545573101102103105109113

2.4. Includes all sources of radiation exposure: background, medical, air travel, present and proposed reactors? 616223551107

2.4.1. Voluntary vs. involuntary nature of radiation exposures highlighted? 56

2.4.2. Explicit section on tritium? 1057585960108115 
2.4.2.1. Differentiates between HTO and OBT? 57585960108115

2.5. Non-radioactive hazardous emissions, e.g., Hydrazine, included in risks to human health? 132251

2.5.1. Baseline concentrations of non-radioactive hazardous emissions established? 119

2.6. Addresses both short-lived and long-lived radionuclides? 1155101123

2.6.1. Geographic and time boundaries justified? 215661

2.6.2. Addresses potential harm to future generations? 411435556107

\section{Evaluation}

2.1 .

2.1.1.

2.1.2.

2.1.3.

2.1.4.

2.1.5.

2.2.

2.3

2.3.1.

2.3.2.

2.3.3.
2.3.4.

2.3.4.1.

2.4 .

2.4.1.

2.4.2.

2.4.2.1.

2.5 .

2.5.1.

2.6

2.6.1.

2.6.2.

Summarize any strengths and weaknesses and state whether this stage was completed sufficiently overall. 


\section{Dose-response Assessment}

3.1. Dose-response data obtained from at least 1 of most recent publication of ICRP/ UNSCEAR/ NAS-BEIR/ HPA/ USEPA/ ECRR/ CERRIE or other sources? 46485162636465

3.1.1. Describes and justifies selected theory of low level radiation model? 68666768 69

3.1.2. States all important health effects from ionizing radiation: chromosomal aberrations, solid cancers, leukemia, hereditary diseases, cardiovascular diseases? 465170717273101

3.1.3. Doses account for different radiosensitivities of males and females? 1032747597

3.1.4. All necessary Dose Conversion Factors included?76 77115123

3.1.5. Different age groups used to calculate doses (e.g., 0-5 years, 6-15 years, 16-70 years)? 611325174101105120

3.1.6. Determines most significant radionuclide(s) for human health? 1929

3.2. Mentions studies relevant to the proposed technology or exposed population/workers (literature review)? 33394356757879104114

3.2.1. Discusses limitations of findings (weakness of ecological studies, small sample size, short follow-up, healthy worker effect etc)? 1235365975104105114

3.3. Differentiates between internal and external exposures and underlying uncertainties? 67105564808182101105

3.3.1. Differences of alpha, beta, gamma radiation explained? 26556277105109

3.3.2. Describes certain radionuclides that can bioaccumulate in certain organs over time? 78758182105

3.3.3. Discusses possibility of synergistic relationships involving ionizing radiation e.g., asbestos exposure, smoking, plutonium in chlorinated drinking water? 2243 758384110

3.4. Predicts magnitude of offsite health consequences to exposed population for all significant accident release categories (i.e., Level 3 PRA)? 920212330325185101 106117119128

3.5. Identifies most significant non-radioactive emission for human health? ${ }^{19}$

\section{Evaluation}

3.1.

3.1.1.

3.1.2.

3.1.3.

3.1.4.

3.1 .5 .

3.1.6.

3.2.
3.2.1.

3.3

3.3.1

3.3.2.

3.3.3.

3.4 .

3.5 . 
Summarize any strengths and weaknesses and state whether this stage was completed sufficiently overall.

\section{Risk Characterization}

4.1. Definition of an acceptable risk defined? 4195767107112

4.1.1. Identification, if necessary, of any specific groups (e.g., workers, infants) that may approach acceptable risk limits? 11425183

4.1.2. Determines if doses for 'a few tens of people' likely to exceed acceptable limits? 112251

4.1.3. Risks below Canadian/international/voluntary HRA standards with justification of any discrepancies? 1986

4.1.4. Risks below Canadian/international/voluntary PRA standards with justification of any discrepancies? 1986

4.2. Addresses perceived risk (radiophobia)? 8101418838788111

4.2.1. Explains possible psychological effects from radiation exposure? 8788126127

4.3. Describes various uncertainties, assumptions and confidence levels? 4810161734 3839518399101112114123128

4.3.1. In all calculations? 3839121

4.3.2. In all models? 8343839128

4.4. Formal environmental monitoring discussed and committed? 183656101114122

4.4.1. Monitoring of health indicators in exposed population/workers proposed? 1710 505190122

4.4.2. Any bio-monitoring of non-human biota to measure internal (alpha, beta) radiation exposures? 344283100101103

4.4.3. Real-time monitoring of environmental radioactivity available to public? 6142251 59

4.5. Commitment to specific formal mitigation measures with evidence of success mentioned? 19333690

4.5.1. Describes reduced health risks due to mitigation? 183340 
4.6. No biases in presentation of material: does not sway reader by undermining effects of ionizing radiation? 363656619192100104

4.6.1. Precautionary principle addressed? 49396100

4.7. Addresses sustainability (definition given?) of project (e.g., use of non-renewable resources)? 413405693107

\section{Evaluation}

4.1.

4.1.1.

4.4 .

4.1.2.

4.4.1.

4.1.3.

4.4.2.

4.1.4.

4.4.3.

4.2.

4.5 .

4.2.1.

4.5.1

4.3.

4.6.

4.3.1.

4.6.1

4.3.2.

4.7.

Summarize any strengths and weaknesses and state whether this stage was completed sufficiently overall. 


\section{Process}

5.1. Broad WHO definition of health accepted? 36404356111122

5.2. Appropriate timeliness of HRA: process started before any action? 36618694

5.3. Communicates risks clearly? 1419107

5.3.1. Non-technical summaries? 1819

5.3.2. Various dose metrics and units defined and kept uniform? 1962

5.4. Evidence of HRA peer review process (e.g., Health Canada)? 61122516194101107

5.4.1. Reviewed by individuals/groups not affiliated with the project? 7225161101

5.5. Evidence of PRA peer review process (e.g., CNSC)? 792061107128

5.5.1. Reviewed by individuals/groups not affiliated with the project? 792061128

5.6. Evidence of range of independent health experts consulted? 473961101107111114

5.7. Early evidence of stakeholder involvement to address health concerns? 47101418 22364356839596111114

5.7.1. Were the most prominent public concerns identified to be addressed in the HRA? 11893107

5.7.2. Activities/results of any workshops disseminated? 3618

5.8. Separate section for HRA? 118

5.9. Funding of any new studies to address ongoing public concerns, e.g. tritium risks? 75693

5.10. Confirms refurbishment would bring reactor up to present international safety standards? 451015117

5.11. Emergency Evacuation Plan guided by highest risk areas as outlined in full Level 3 PRA? 2124125128

5.12. If $E A$ is a panel review, independent reviewers selected (i.e., not affiliated with nuclear industry? 6198

\section{Evaluation}

5.1.

5.2.

5.3.

5.3.1.

5.3.2.

5.4 .

5.4.1.

5.5.

5.5.1

5.6 .
5.6.

5.7.

5.7.1.

5.7.2.

5.8 .

5.9 .

5.10 .

5.11 .

5.12. 
Summarize any strengths and weaknesses and state whether this stage was completed sufficiently overall.

Summarize any strengths and weaknesses of the HRA and state whether the document is sufficient or deficient, given the provided information.

\section{Sufficient / Deficient}




\section{Endnotes}

1 Demidova, O., \& Cherp, A. 2005. Risk assessment for improved treatment of health considerations in EIA. Environmental Impact Assessment Review. 25(4): 411-429.

2 WHO. 1994. Nuclear power and health: The implications for health of nuclear power production. WHO Regional Publication European Series, No:51:Copenhagen.

3 Oxman, A.D., Shannon, H.S., Garland, W.J. and Torrance, G.W. 1989. Nuclear safety in Ontario: A critical review of quantitative analyses. Risk Analysis. 9(1):43-54.

${ }^{4}$ O'Connor, M. and van den Hove, S. 2001. Prospects for public participation on nuclear risks and policy options: Innovations in governance practices for sustainable development in the European Union. Journal of Hazardous Materials. 86:77-99.

${ }^{5}$ Lochbaum, D. 2004. U.S. nuclear plants in the 21st century: The risk of at lifetime. Report prepared for Union of Concerned Scientists.

6 Interviewee \#1 per. comm., 2009.

7 Interviewee \#2 per. comm., 2009.

8 Interviewee \#3 per. comm., 2009.

9 Interviewee \#5 per. comm., 2009.

10 Interviewee \#6 per. comm., 2009.

11 ICRP 101. 2006. Assessing dose of the representative person for the purpose of radiation protection of the public and the optimization of radiological protection:

Broadening the process. Volume 36, 3.

12 Dodic-Fikfak, M., Clapp, R. and Kriebel, D. 1999. The health risks of decommissioning nuclear facilities. New Solutions. 9(2):153-161.

13 Winfield, M., Jamison, A., Wong, R. and Czajkowski, P. 2006. Nuclear Power in Canada: An examination of risks, impacts and sustainability. The Pembina Institute.

14 Interviewee \#12 per. comm., 2009

15 Hirsch, H., Becker, O., Schneider, M. and Froggatt, A. 2008. Nuclear reactor hazards: ongoing dangers of operating nuclear technology in the 21st century. Estudos Avancados. 21(59):2007.

16 Paustenbach, D.J. 2000. The practice of exposure assessment: A state-of-the-art review. Journal of Toxicology and Environmental Health, Part B. 3:179-291. 
17 von Stackelberg, K. and Burmaster, D.E. 1994. A discussion on the use of probabilistic risk assessment in human health impact assessment. Environ Impact Assessment Review. 14:385-401.

${ }^{18}$ British Medical Association (BMA). 1998. Health and environmental impact assessment: An integrated approach. London:Earthscan publications.

19 Lee, N., Colley, R., Bonde, J. and Simpson, J. 1999. Reviewing the quality of environmental statements and environmental appraisals, Occasional Paper Number 55. Manchester: Department of Planning and Landscape, University of Manchester,

20 Thompson, G.R. 2000. A review of the accident risk posed by the Pickering $A$ Nuclear Generating Station. A report to the standing committee on energy, environment and natural resources of the Canadian Senate.

${ }^{21}$ The Standing Senate Committee on Energy, The Environment and Natural Resources. 2001. Canada s nuclear reactors: How much safety is enough?. Interim Report.

22 Interviewee \#4 per. comm., 2009

23 U.S. Nuclear Regulatory Commission (USNRC). 1990. Severe accident risks: An assessment for five U.S. nuclear power plants, final summary report. NUREG-1150. Washington, D.C.

${ }^{24}$ Cameron, R.F. and Willers, A. 2001. Use of risk assessment in the nuclear industry with specific reference to the Australian situation. Reliability Engineering and System Safety. 74:275-282.

25 Garrick, B.J. and Christie, R.F. 2002. Probabilistic risk assessment practices in the USA for nuclear power plants. Safety Science. 40:177-201.

${ }^{26}$ Thompson, G.R. 2008a. Scope of the environmental impact statement for new nuclear power plants at the Bruce site in Ontario: Assessment of accidents and malfunctions. Prepared by the Institute for Resource and Security Studies for Greenpeace Canada, June 2008.

27 Thompson, G.R. 2008b. Scope of the EIS for new nuclear power plants at the Darlington site in Ontario: Accidents, malfunctions and the precautionary approach. Prepared by the Institute for Resource and Security Studies for Greenpeace Canada, November 2008.

29 Slaper, H. and Blaauboer, R. 1998. A probabilistic risk assessment for accidental releases from nuclear power plants in Europe. Journal of Hazardous Materials. 61:209-215. 
30 Mueller, C.J. 1996. Facilitating relative comparisons of health impacts from postulated accidents in environmental impact statements. Argonne National Laboratory.

31 Till, J.E. and Grogan, H.A. (Eds.) 2008. Radiological Risk Assessment and Environmental Analysis. New York: Oxford University Press.

32 Adler, M.A. 2004. Against 'individual risk': A sympathetic critique of risk assessment. University of Pennsylvania Law Review. 153:1121-1250.

${ }^{33}$ Bhatia, R. 2007. Protecting health using an environmental impact assessment: A case study of San Francisco Land Use Decisionmaking. American Journal of Public Health. 97(3):406-413.

${ }^{34}$ Nieuwenhuijsen, M., Paustenbach and Duarte-Davidson. 2006. New developments in exposure assessment: The impact on the practice of health risk assessment and epidemiological studies. Environment International. 32:996-1009.

35 Muirhead, C.R. 2008. Exposure assessment: Implications for epidemiological studies of ionizing radiation. Radiation Protection Dosimetry. 132(2):134-138.

${ }^{36}$ Parry, J.M., Kemm, J.R. 2005. Criteria for use in the evaluation of health impact assessments. Public Health. 119:1122-1129.

37 Cole, B.L. \& Fielding, J.E. 2007. Health Impact Assessment: A tool to help policy makers understand health beyond health care. Annual Review of Public Health. 28: 393-412.

38 Zou, B., Wilson, J.G., Zhan, F.B. and Zeng, Y. 2009. Air pollution exposure assessment methods utilized in epidemiological studies. J. Environ. Monit. 11:475-490.

39 Briggs, D.J., Sabel, C.E. and Lee, K. 2009. Uncertainty in epidemiology and health risk and impact assessment. Environ Geochem Health. 31:189-203.

40 Health Canada. 2004a. Canadian handbook on health impact assessment Volume 1:The basics. Ottawa: Government of Canada.

41 O'Keefe, E. and Scott-Samuel. 2002. Human rights and wrongs: Could health impact assessment?. The Journal of Law, Medicine \& Ethics. 30(4):734-738.

42 USEPA. 2003. Framework for Cumulative Risk Assessment. EPA/600/P-02/001F. Washington:U.S. Environmental Protection Agency, Office of Research and Development.

43 Davies, K. and Sadler, B. 1997. Environmental Assessment and Human Health: Perspectives, Approaches, and Future Directions. Ottawa: Health Canada. 
${ }^{44}$ Chen, J. and Moir, D. 2009. On the need for a radiation accident registry in Canada. Radiation Protection Dosimetry. 134(3-4):181-183.

45 Kaatsch, P., Spix, C., Schulze-Rath, R., Schmiedel, S.and Blettner, M. 2008. Leukaemia in young children living in the vicinity of German nuclear power plants. International Journal of Cancer. 1220:721-726.

46 National Research Council. 2005. Committee to assess health risks from exposure to low levels of ionizing radiation (BEIR VII). Washington:National Academic Press.

47 Mangano, J.J., Gould, J.M., Sternglass, E.J., Sherman, J.D., Brown, J. and McDonnell, W. 2002. Infant death and childhood cancer reductions after nuclear plant closings in the United States. Archives of Environmental Health. 51(1):23-31.

48 United Nations Scientific Committee on the Effects of Atomic Radiation (UNSCEAR). 2000. Sources and effects of ionizing radiation. Volume I, Sources. UNSCEAR 2000 Report to the General Assembly, with scientific annexes. New York:United Nations.

49 Petticrew, M., Cummins, S., Sparks, L. and Findlay, A. 2007. Validating health impact assessment: prediction is difficult (especially about the future). Environmental Impact Assessment Review. 27:101-107.

50 Veerman, J.L., Mackenbach, J.P. and Barendregt, J.J. 2007. Validity of predictions in health impact assessment. Journal of Epidemiology and Community Health.

61:362-366.

51 Interviewee \#9 per. comm., 2009

52 Spiegel, J. \& Yassi, A. 1997. The use of health indicators in environmental assessment. Journal of Medical Systems. 21(5):275-289.

53 Schrader-Frechette, K. 2007. Trimming exposure data, putting radiation workers at risk: Improving disclosure and consent through a national dose registry. American Journal of Public Health. 97(9):.

54 Wing, S., Richardson, D. and Stewart, A. 1999. The relevance of occupational epidemiology to radiation protection standards. New Solutions. 9(2):133-151.

55 Interviewee \#10 per. comm., 2009

56 Quigley, R., den Broeder, L., Furu, P., Bond, A., Cave, B. and Bos, R. 2006. Health Impact Assessment International Best Practice Principles. Special Publication Series No. 5. Fargo:International Association for Impact Assessment. 
57 McMullan, C. and Eyles, J. 1999. Risky Business: An analysis of claimsmaking in the development of an Ontario drinking water objective for tritium. Social Problems. 46(2): 294-311.

58 Fairlie, I. 2008. The hazards of tritium - revisited. Medicine, Conflict and Survival. 24 (4):306-319.

59 Fairlie, I. 2007a. Tritium hazard report: pollution and radiation risk from Canadian nuclear facilities. Greenpeace Canada. June 2007.

60 Little, M.P and Wakeford, R. 2008. Systematic review of epidemiological studies of exposure to tritium. Journal of Radiological Protection. 28:9-32.

61 Birley, M. 2003. Health Impact Assessment, integration and critical appraisal. Impact Assessment and Project Appraisal. 21(4):313-321.

62 Robson, M.G. and Toscano, W.A. (Eds.) 2007. Risk Assessment for Environmental Health. San Francisco: Jossey-Bass.

63 Eckerman, K.F., Legett, R., Nelson, C., Puskin, J. and Richardson, A. 1999. Cancer risk coefficients for environmental exposure to radionuclides: Federal Guidance Report No. 13. United States Environmental Protection Agency (USEPA).

64 Committee Examining Radiation Risks from Internal Emitters (CERRIE). 2004. Reports of the Committee Examining Radiation Risks from Internal Emitters. Chilton: National Radiological Protection Board.

65 European Committee on Radiation Risk. (ECRR) 2003. The health effects of ionizing radiation exposure at low doses and low dose rates for radiation protection purposes: Regulators edition. Brussels:Green Audit.

66 Brenner, D.J. and Sachs, R. K. 2006. Estimating radiation-induced cancer risks at very low doses: rationale for using a linear no-threshold approach. Radiat Environ Biophys. 44:253-256.

67 Moore, L. 2002. Lowering the bar. Bulletin of Atomic Scientists. 58(3):28-37.

68 Nussbaum, R.H. 1998. The linear no-threshold dose-effect relation: Is it relevant to radiation protection regulation?. Medical Physics. 25(3): 291-299.

69 Kohnlein, W. and Nussbaum, R.H. 1998. False alarm or public health hazard?: Chronic low-dose external radiation exposure. Medicine and Global Survival. 5(1): 14-21. 
70 Busby, C., Lengfelder, E., Pflugbeil, S. and Schmitz-Feuerhake, I. 2009. The evidence of radiation effects in embryos and fetuses exposed to Chernobyl fallout and the question of dose response. Medicine, Conflict and Survival. 25(1):20-40.

71 Richardson, R.B. 2009. Factors that elevate the internal radionuclide and chemical retention, dose and health risks to infants and children in a radiological-nuclear emergency. Radiation Protection Dosimetry. 134(3-4):167-180.

72 Zielinski, J.M. et al. 2009. Low dose ionizing radiation exposure and cardiovascular disease mortality: Cohort study based on Canadian national dose registry of radiation workers. International Journal of Occupational Medicine and Environmental Health. 22 (1):27-33.

73 Ritz, B., Morgernstern, H., Froines, J. and Young, B.B. 1999. Effects of exposure to external ionizing radiation on cancer mortality in nuclear workers monitored for radiation at Rocketdyne/Atomics International. American Journal of Industrial Medicine. 35:21-31.

74 Makhijani, A. 2008. The use of the reference man in radiation protection standards and guidance with recommendations for change. Report prepared for the Institute for Energy and Environmental Research, December, 2008.

75 Richardson, D., Wing, S. and Stewart, A. 1997. Epidemiologic studies of the effects of exposure to ionizing radiation. Schleswig-Holstein: Ministry of Finance and Energy.

76 Fairlie, I. 2007b. RBE and wR values of Auger emitters and low-range beta emitters with particular reference to tritium. Journal of Radiological Protection. 27:157-168.

77 Harrison, J. and Day, P. 2008. Radiation doses and risks from internal emitters. Journal of Radiological Protection. 28:137-159.

$78 \mathrm{WHO}, 2000$. Evaluation and use of epidemiological evidence for environmental health risk assessment: Guideline Document. Denmark: Regional Office for Europe.

79 Health Canada. 2004c. Canadian handbook on health impact assessment Volume 3: The multidisciplinary team. Ottawa: Government of Canada.

80 Sawada, S. 2007. Cover-up of the effects of internal exposure by residual radiation from atomic bombing of Hiroshima and Nagasaki. Medicine, Conflict and Survival. 23 (1):58-74.

81 Brown, S.C., Schonbeck, M.F., McClure, D., Baron, A.E., Navidi, W.C., Byers, T. et al. 2004. Lung cancer and internal doses among plutonium workers at the Rocky Flats plant: A case-control study. American Journal of Epidemiology. 160(2):163-172.

82 Tokarskaya, Z.B., Zhuntova, G.V., Scott, B.R., Khokhryakov, V.F., Belyaeva, Z.D., Vasilenko, E.K. et al. 2006. Influence of alpha and gamma radiations and non-radiation 
risk factors on the incidence of malignant liver tumors among Mayak PA workers. Health Physics. 91(4):296-310.

${ }^{83}$ Callahan, M.A. and Sexton, K. 2007. If cumulative risk assessment is the answer, what is the question?. Environmental Health Perspectives. 115(5):799-806.

${ }^{84}$ Busby, C. 2005. Does uranium contamination amplify natural background radiation dose to the DNA?. Eur. J. Biol. Bioelectromagn. 1:120-131.

85 Rigina, O. and Baklanov, A. 2002. Regional radiation risk and vulnerability assessment by integration of mathematical modelling and GIS analysis. Environment International. 27:527-540.

86 Bekker, M.P.M., Putters, K., van der Grinten, T.E.D. 2005. Evaluating the impact of HIA on urban reconstruction decision-making. Who manages whose risks?. Environmental Impact Assessment Review. 25:758-771.

87 Slovic, P. 1996. Perception of risk from radiation. Radiation Protection Dosimetry. 68 (3/4):165-180.

88 METROPOLITAN EDISON CO. V. PANE, 460 U. S. 766. 1983.

89 Mangano, J.J. 2006. A short latency between radiation exposure from nuclear plants and cancer in young children. International Journal of Health Services. 36(1):113-135.

90 Bhatia, R. and Wernham, A. 2008. Integrating human health into environmental impact assessment: An unrealized opportunity for environmental health and justice. Environmental Health Perspectives. 116(8):991-1000.

91 Wing, S. 2003. Objectivity and ethics in environmental health science. Environmental Health Perspectives. 111(14):1809-1818.

92 Bailar, J.C. 2006. How to distort the scientific record without actually lying: truth, and the arts of science. European Journal of Oncology. 11(4):217-224.

${ }^{93}$ Health Canada. 2004b. Canadian handbook on health impact assessment Volume 2: Approaches and Decision Making. Ottawa: Government of Canada.

${ }^{94}$ Kemm, J., Parry, J. and Palmer, S. 2004. Health impact assessment: concepts, theory, techniques, and applications. Oxford: Oxford University Press.

95 Campbell-Mohn, C. \& Applegate, J.S. 1999. Learning from NEPA: guidelines for responsible risk legislation. Harvard Environmental Law Review. 23:93-139.

96 Jardine, C.G. et al. 2003. Risk management frameworks for human health and environmental risks. Journal of Toxicology and Environmental Health. 6:569-641. 
97 Wilkinson, D. 2009. Dealing with at-risk populations in radiological/nuclear emergencies. Radiation Protection Dosimetry. 134(3-4):136-142.

98 Huff, J.H. 2007. Industry influence on occupational and environmental public health. International Journal of Occupational and Environmental Health. 13:107-117.

99 Burmaster, D.E. and Anderson, P.D. 1994. Principles of good practice for the use of Monte Carlo techniques in human health and ecological risk assessments. Risk Analysis. 14(4):477-481.

100 Nussbaum, R. 2007. The Chernobyl nuclear catastrophe: Unacknowledged health detriment. Environmental Health Perspectives. 115(5):238-239.

101 Interviewee \#7 per. comm., 2009.

102 Bondarenko, O.O., Melnychuk, D.V. and Medvedev, S.Y. 2003. Limitation of individual internal exposure by consideration of the confidence interval in routine personal dosimetry at the Chernobyl Sarophagus. Radiation Protection Dosimetry. 107 (4):253-258.

103 Franck, D. 2007. Potential of modern technologies for improving internal exposure monitoring. Radiation Protection Dosimetry. 127(1-4):233-239.

104 Nussbaum, R.H. 2007. Manipulating public health research: The nuclear and radiation health establishments. Int $J$ Occup Health. 13:328-330.

105 Nussbaum, R.H. 2009. Childhood leukemia and cancers near German nuclear reactors: Significance, context, and ramifications of recent studies. Int J Occup Health. 15:318-323.

106 Alzbutas, R. and Maioli, A. 2008. Risk zoning in relation to risk of external events (applications to IRIS design). Int. J. Risk Assessment and Management. 8(1/2):104-122.

107 Bredimas, A. and Nuttall, W.J. 2008. An international comparison of regulatory organizations and licensing procedures for new nuclear power plants. Energy Policy. 36:1344-1354.

108 Galeriu, D., Paunescu, N., Mocanu, N. and Melintescu, A. 2003. A data base for tritium level around a chronic source, Report WP3 IDRANAP 58-03/2003. European Commission Center of Excellence IDRANAP.

109 Le Guen, B., Roupioz, A., Rabus, B., Bouvy, A., Labouglie, J.F. and Garcier, Y. 2003. Control of the risk of exposure to alpha emitting radionuclides in French nuclear power plants: Example of Cattenom. Radiation Protection Dosimetry. 105(1-4):303-309. 
110 UNSCEAR. 2000. Sources and effects of ionizing radiation, Volume II: Effects, Annex $H$ : Combined effects of radiation and other agents. New York: United Nations.

111 Mindell, J.S., Boltong, A. and Forde, I. 2008. A review of health impact assessment frameworks. Public Health. 122:1177-1187.

112 Canadian Environmental Assessment Research Council. 1992. Human health and environmental assessment: $A$ research prospectus. Ottawa: CEARC.

${ }^{113}$ Alm-Lytz, K., Riihiluoma, V. and Hyvonen, H. 2001. Occupational exposures of nuclear power plant workers in Finland. Radiation Protection Dosimetry. 96(1-4):39-42.

${ }^{114}$ Cole, B.L., Shimkhada, R., Fielding, J.E., Kominski, G. and Morgernstern, H. 2005. Methodologies for realizing the potential of health impact assessment. American Journal of Preventative Medicine. 28(4):382-389.

115 Harrison, J.D., Khursheed, A. and Lambert, B.E. 2002. Uncertainties in dose coefficients for intakes of tritiated water and organically bound forms of tritium by members of the public. Radiation Protection Dosimetry. 98(3):299-311.

116 Canadian Nuclear Safety Commission. 2008. Site evaluation for new nuclear power plants, RD-346. Regulatory Document. CNSC.

117 Thompson, G.R. 2008c. Risks of operating CANDU 6 nuclear power plants: Gentilly unit 2 refurbishment and its global implications. Prepared for Greenpeace Canada, 2008.

118 Hayns, M.R. 1999. The evolution of probabilistic risk assessment in the nuclear industry. Trans IChemE. 77(B):117-142.

119 Sues, R.H., Amico, P.J. and Campbell, R.D. 1990. Significance of earthquake risk in nuclear power plant probabilistic risk assessments. Nuclear Engineering and Design. 123:27-44.

120 Finley, B., Proctor, D., Scott, P., Harrington, N., Paustenbach, D. and Price, P. 1994. Recommended distribution for exposure factors frequently used in health risk assessment. Risk Analysis. 14(4):533-553.

121 Nuclear Energy Agency (NEA), Committee on the Safety of Nuclear Installations. 2009. Probabilistic safety analysis (PSA) of other external events than earthquake, $N E A / C S N I / R(2009) 4$, March.

122 Hansell, A. and Aylin, P. 2003. Use of health data in health impact assessment. Impact Assessment and Project Appraisal. 21(1):57-64. 
123 Salbu, B, Lind, O.C. and Skipperud, L. 2004. Radionuclide speciation and its relevance in environmental impact assessments. Journal of Environmental Radioactivity. 74:233-242.

124 Kirchsteiger, C. 2006. Current practices for risk zoning around nuclear power plants in comparison to other industry sectors. Journal of Hazardous Materials. 136:392-397.

125 Zandvoort, H. 2008. Risk zoning and risk decision making. International Journal of Risk Assessment and Management. 8(1/2):3-18.

${ }^{126}$ Baverstock, K. and Williams, D. 2006. The Chernobyl accident 20 years on: An assessment of the health consequences and the international response. Environmental Health Perspectives. 114(9):1312-1317.

127 Cwikel, J.G., Gidron, Y. and Quastel, M. 2010. Low-dose environmental radiation, DNA damage, and cancer: The possible contribution of psychological factors.

Psychology, Health \& Medicine. 15(1):1-16.

128 Serbanescu, D. 2008. Sensitivity and uncertainty issues in the integrated PRA studies. International Journal of Risk Assessment and Management. 10(1/2):31-56. 